\title{
Search for pair production of up-type vector-like quarks and for four-top-quark events in final states with multiple $b$-jets with the ATLAS detector
}

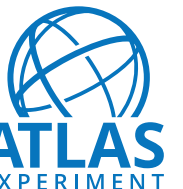

\section{The ATLAS collaboration}

E-mail: atlas.publications@cern.ch

ABstRACT: A search for pair production of up-type vector-like quarks $(T)$ with a significant branching ratio into a top quark and either a Standard Model Higgs boson or a $Z$ boson is presented. The same analysis is also used to search for four-top-quark production in several new physics scenarios. The search is based on a dataset of $p p$ collisions at $\sqrt{s}=$ $13 \mathrm{TeV}$ recorded in 2015 and 2016 with the ATLAS detector at the CERN Large Hadron Collider and corresponds to an integrated luminosity of $36.1 \mathrm{fb}^{-1}$. Data are analysed in the lepton+jets final state, characterised by an isolated electron or muon with high transverse momentum, large missing transverse momentum and multiple jets, as well as the jets $+E_{\mathrm{T}}^{\text {miss }}$ final state, characterised by multiple jets and large missing transverse momentum. The search exploits the high multiplicity of jets identified as originating from $b$-quarks, and the presence of boosted, hadronically decaying top quarks and Higgs bosons reconstructed as large-radius jets, characteristic of signal events. No significant excess above the Standard Model expectation is observed, and 95\% CL upper limits are set on the production cross sections for the different signal processes considered. These cross-section limits are used to derive lower limits on the mass of a vector-like $T$ quark under several branching ratio hypotheses assuming contributions from $T \rightarrow W b, Z t, H t$ decays. The $95 \%$ CL observed lower limits on the $T$ quark mass range between $0.99 \mathrm{TeV}$ and $1.43 \mathrm{TeV}$ for all possible values of the branching ratios into the three decay modes considered, significantly extending the reach beyond that of previous searches. Additionally, upper limits on anomalous four-topquark production are set in the context of an effective field theory model, as well as in an universal extra dimensions model.

KEywords: Beyond Standard Model, Hadron-Hadron scattering (experiments), vectorlike quarks

ARXIV EPRINT: 1803.09678 


\section{Contents}

1 Introduction 1

2 ATLAS detector $\quad 4$

3 Object reconstruction $\quad 5$

4 Data sample and event preselection $\quad 7$

5 Signal and background modelling $\quad \mathbf{8}$

5.1 Signal modelling 8

$\begin{array}{llr}5.2 & \text { Background modelling } & 9\end{array}$

$\begin{array}{llr}6 & \text { Search strategy } & 12\end{array}$

7 Systematic uncertainties $\quad 16$

$\begin{array}{lll}7.1 \text { Luminosity } & 19\end{array}$

$\begin{array}{lll}7.2 & \text { Reconstructed objects } & 20\end{array}$

$\begin{array}{lll}7.3 & \text { Background modelling } & 22\end{array}$

8 Statistical analysis $\quad 25$

9 Results $\quad 26$

9.1 Likelihood fits to data 26

9.2 Limits on vector-like quark pair production 28

$\begin{array}{lll}9.3 & \text { Limits on four-top-quark production } 38\end{array}$

10 Conclusion $\quad 42$

$\begin{array}{ll}\text { The ATLAS collaboration } & 51\end{array}$

\section{Introduction}

The discovery of a new particle consistent with the Standard Model (SM) Higgs boson by the ATLAS [1] and CMS [2] experiments at the Large Hadron Collider (LHC) represents a milestone in high-energy physics. A comprehensive programme of measurements of the Higgs boson properties to unravel its nature is underway at the LHC, so far yielding results compatible with the SM predictions. This makes it more urgent than ever before to provide an explanation for why the electroweak mass scale (and the Higgs boson mass along with it) is so small compared to the Planck scale, a situation known as the hierarchy problem. Naturalness arguments [3] require that quadratic divergences that arise from radiative 
corrections to the Higgs boson mass are cancelled out by some new mechanism in order to avoid fine-tuning. To that effect, several explanations have been proposed in theories beyond the SM (BSM).

One such solution involves the existence of a new strongly interacting sector, in which the Higgs boson would be a pseudo-Nambu-Goldstone boson [4] of a spontaneously broken global symmetry. One particular realisation of this scenario, referred to as Composite Higgs $[5,6]$, addresses many open questions in the SM, such as the stability of the Higgs boson mass against quantum corrections, and the hierarchy in the mass spectrum of the SM particles, which would be explained by partial compositeness. In this scenario, the top quark would be a mostly composite particle, while all other SM fermions would be mostly elementary. A key prediction is the existence of new fermionic resonances referred to as vector-like quarks, which are also common in many other BSM scenarios. Vector-like quarks are defined as colour-triplet spin- $1 / 2$ fermions whose left- and right-handed chiral components have the same transformation properties under the weak-isospin $\mathrm{SU}(2)$ gauge group [7, 8]. Depending on the model, vector-like quarks are classified as $\mathrm{SU}(2)$ singlets, doublets or triplets of flavours $T, B, X$ or $Y$, in which the first two have the same charge as the SM top and bottom quarks while the vector-like $Y$ and $X$ quarks have charge $-4 / 3 e$ and $5 / 3 e$. In addition, in these models, vector-like quarks are expected to couple preferentially to third-generation quarks [7,9] and can have flavour-changing neutral-current decays in addition to the charged-current decays characteristic of chiral quarks. As a result, an uptype $T$ quark can decay not only into a $W$ boson and a $b$-quark, but also into a $Z$ or Higgs boson and a top quark $(T \rightarrow W b, Z t$, and $H t)$. Similarly, a down-type $B$ quark can decay into a $Z$ or Higgs boson and a $b$ quark, in addition to decaying into a $W$ boson and a top quark $(B \rightarrow W t, Z b$ and $H b)$. Vector-like $Y$ quarks decay exclusively into $W b$ and vectorlike $X$ quarks decay exclusively into $W t$. To be consistent with the results from precision electroweak measurements a small mass-splitting between vector-like quarks belonging to the same $\mathrm{SU}(2)$ multiplet is required, but no requirement is placed on which member of the multiplet is heavier [10]. At the LHC, vector-like quarks with masses below $\sim 1 \mathrm{TeV}$ would be predominantly produced in pairs via the strong interaction. For higher masses, single production, mediated by the electroweak interaction, may dominate depending on the coupling strength of the interaction between the vector-like quark and the SM quarks.

Another prediction of the Composite Higgs paradigm, as well as other BSM scenarios, such as Randall-Sundrum extra dimensions, is the existence of new heavy vector resonances, which would predominantly couple to the third-generation quarks and thus lead to enhanced four-top-quark production at high energies [11-15]. In particular, the class of models where such vector particles are strongly coupled to the right-handed top quark are much less constrained by precision electroweak measurements than in the case of couplings to the left-handed top quark [16]. In the limit of sufficiently heavy particles, these models can be described via an effective field theory (EFT) involving a four-fermion contact interaction [17]. The corresponding Lagrangian is

$$
\mathcal{L}_{4 t}=\frac{\left|C_{4 t}\right|}{\Lambda^{2}}\left(\bar{t}_{\mathrm{R}} \gamma^{\mu} t_{\mathrm{R}}\right)\left(\bar{t}_{\mathrm{R}} \gamma_{\mu} t_{\mathrm{R}}\right)
$$


where $t_{\mathrm{R}}$ is the right-handed top quark spinor, $\gamma_{\mu}$ are the Dirac matrices, $C_{4 t}$ is the coupling constant, and $\Lambda$ is the energy scale above which the effects of direct production of new vector particles must be considered. Anomalous four-top-quark production also arises in Universal Extra Dimensions (UED) models, which involve new heavy particles. For instance, in an UED model with two extra dimensions that are compactified using the geometry of the real projective plane (2UED/RPP) [18], the momenta of particles are discretised along the directions of the extra dimensions. A tier of Kaluza-Klein (KK) towers is labelled by two integers, $k$ and $\ell$, referred to as "tier $(k, \ell)$ ". Within a given tier, the squared masses of the particles are given at leading order by $m^{2}=k^{2} / R_{4}^{2}+\ell^{2} / R_{5}^{2}$, where $\pi R_{4}$ and $\pi R_{5}$ are the sizes of the two extra dimensions. The model is parameterised by $R_{4}$ and $R_{5}$ or, alternatively, by $m_{\mathrm{KK}}=1 / R_{4}$ and $\xi=R_{4} / R_{5}$. Four-top-quark production can arise from tier $(1,1)$, where particles from this tier have to be pair produced because of symmetries of the model. Then they chain-decay into the lightest particle of this tier, the heavy photon $A^{(1,1)}$, by emitting SM particles. The branching ratios of $A^{(1,1)}$ into SM particles are not predicted by the model, although the decay into $t \bar{t}$ is expected to be dominant [19].

This paper presents a search for $T \bar{T}$ production with at least one $T$ quark decaying into $H t$ with $H \rightarrow b \bar{b}$, or into $Z t$ with $Z \rightarrow \nu \bar{\nu}$, as well as for anomalous four-top-quark production within an EFT model and within the 2UED/RPP model (see figure 1). Recent searches for $T \bar{T}$ production have been performed by the ATLAS [20, 21] and CMS [22, 23] collaborations using up to $36.1 \mathrm{fb}^{-1}$ of $p p$ collisions at $\sqrt{s}=13 \mathrm{TeV}$. The most restrictive $95 \% \mathrm{CL}$ lower limits on the $T$ quark mass obtained are $1.35 \mathrm{TeV}$ and $1.16 \mathrm{TeV}$, corresponding to branching ratio assumptions of $\mathcal{B}(T \rightarrow W b)=1$ and $\mathcal{B}(T \rightarrow Z t)=1$, respectively. Previous searches for anomalous $t \bar{t} t \bar{t}$ production have been performed by the ATLAS Collaboration using the full Run-1 dataset [24, 25], where 95\% CL limits of $\left|C_{4 t}\right| / \Lambda^{2}<6.6 \mathrm{TeV}^{-2}$ and $m_{K K}>1.1 \mathrm{TeV}$ were obtained in the case of the EFT and the 2UED/RPP models, respectively. A recent search by the CMS Collaboration [26] using $35.9 \mathrm{fb}^{-1}$ of $p p$ collisions at $\sqrt{s}=13 \mathrm{TeV}$ has set an upper limit of $41.7 \mathrm{fb}$ on the SM $t \bar{t} t \bar{t}$ production cross section, about 4.5 times the SM prediction, thus placing some constraints on anomalous production with kinematics like in the SM.

This search uses $36.1 \mathrm{fb}^{-1}$ of data at $\sqrt{s}=13 \mathrm{TeV}$ recorded in 2015 and 2016 by the ATLAS Collaboration, and it closely follows the strategy developed in Run 1 [25], although it incorporates new ingredients, such as the identification of boosted objects, to substantially enhance sensitivity for heavy resonances. Data are analysed in the lepton+jets final state, characterised by an isolated electron or muon with high transverse momentum, large missing transverse momentum and multiple jets and, for the first time in searches for vector-like quarks, also in the jets $+E_{\mathrm{T}}^{\text {miss }}$ final state, characterised by multiple jets and large missing transverse momentum. 


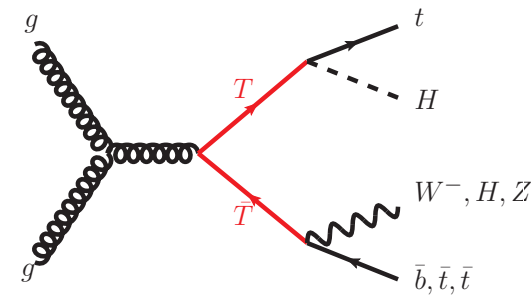

(a)

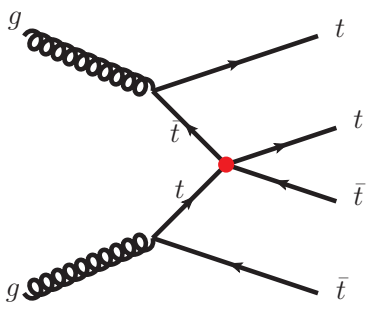

(b)

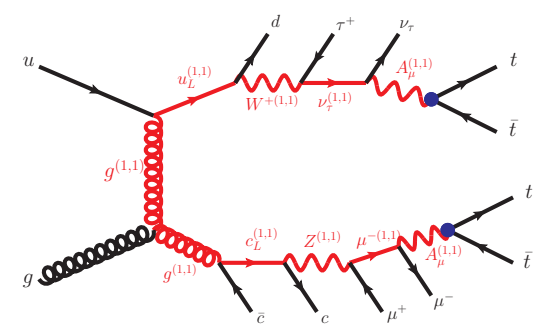

(c)

Figure 1. Representative leading-order Feynman diagrams for the signals probed by this search: (a) $T \bar{T}$ production, and (b) four-top-quark production via an effective four-top-quark interaction in an effective field theory model, and (c) four-top-quark production via cascade decays from KaluzaKlein excitations in a universal extra dimensions model with two extra dimensions compactified using the geometry of the real projective plane.

\section{ATLAS detector}

The ATLAS detector [27] at the LHC covers almost the entire solid angle around the collision point,${ }^{1}$ and consists of an inner tracking detector surrounded by a thin superconducting solenoid producing a $2 \mathrm{~T}$ axial magnetic field, electromagnetic and hadronic calorimeters, and a muon spectrometer incorporating three large toroid magnet assemblies. The inner detector consists of a high-granularity silicon pixel detector, including the insertable B-layer [28], installed in 2014, and a silicon microstrip tracker, together providing a precise reconstruction of tracks of charged particles in the pseudorapidity range $|\eta|<2.5$, complemented by a transition radiation tracker providing tracking and electron identification information for $|\eta|<2.0$. The calorimeter system covers the pseudorapidity range $|\eta|<4.9$. Within the region $|\eta|<3.2$, electromagnetic (EM) calorimetry is provided by barrel and endcap high-granularity lead/liquid-argon (LAr) electromagnetic calorimeters, with an additional thin LAr presampler covering $|\eta|<1.8$, to correct for energy loss in material upstream of the calorimeters. Hadronic calorimetry is provided by a steel/scintillator-tile calorimeter, segmented into three barrel structures within $|\eta|<1.7$, and two copper/LAr hadronic endcap calorimeters. The solid angle coverage is completed with forward copper/LAr and tungsten/LAr calorimeter modules optimised for electromagnetic and hadronic measurements, respectively. The muon spectrometer measures the trajectories of muons with $|\eta|<2.7$ using multiple layers of high-precision tracking chambers located in a toroidal field of approximately $0.5 \mathrm{~T}$ and $1 \mathrm{~T}$ in the central and endcap regions of ATLAS, respectively. The muon spectrometer is also instrumented with separate trigger chambers covering $|\eta|<2.4$. A two-level trigger system [29], consisting of a

\footnotetext{
${ }^{1}$ ATLAS uses a right-handed coordinate system with its origin at the nominal interaction point (IP) in the centre of the detector. The $x$-axis points from the IP to the centre of the LHC ring, the $y$-axis points upward, and the $z$-axis coincides with the axis of the beam pipe. Cylindrical coordinates $(r, \phi)$ are used in the transverse plane, $\phi$ being the azimuthal angle around the beam pipe. The pseudorapidity is defined in terms of the polar angle $\theta$ as $\eta=-\ln \tan (\theta / 2)$. Angular distance is measured in units of $\Delta R \equiv \sqrt{(\Delta \eta)^{2}+(\Delta \phi)^{2}}$.
} 
hardware-based Level-1 trigger followed by a software-based High-Level Trigger (HLT), is used to reduce the event rate to a maximum of around $1 \mathrm{kHz}$ for offline storage.

\section{Object reconstruction}

Interaction vertices from the proton-proton collisions are reconstructed from at least two tracks with transverse momentum $\left(p_{\mathrm{T}}\right)$ larger than $400 \mathrm{MeV}$ that are consistent with originating from the beam collision region in the $x-y$ plane. If more than one primary vertex candidate is found, the candidate whose associated tracks form the largest sum of squared $p_{\mathrm{T}}[30]$ is selected as the hard-scatter primary vertex.

Electron candidates $[31,32]$ are reconstructed from energy clusters in the EM calorimeter that are matched to reconstructed tracks in the inner detector and have $p_{\mathrm{T}}>30 \mathrm{GeV}$ and $\left|\eta_{\text {cluster }}\right|<2.47$; candidates in the transition region between the EM barrel and endcap calorimeter $\left(1.37<\left|\eta_{\text {cluster }}\right|<1.52\right)$ are excluded. They are also required to satisfy the "tight" likelihood-based identification criteria [31] based on calorimeter, tracking and combined variables that provide separation between electrons and jets. Muon candidates [33] are reconstructed by matching track segments in different layers of the muon spectrometer to tracks found in the inner detector. The resulting muon candidates are refitted using the complete track information from both detector systems and are required to have $p_{\mathrm{T}}>30 \mathrm{GeV}$ and $|\eta|<2.5$. Electron (muon) candidates are matched to the primary vertex by requiring that the significance of their transverse impact parameter, $d_{0}$, satisfies $\left|d_{0} / \sigma\left(d_{0}\right)\right|<5(3)$, where $\sigma\left(d_{0}\right)$ is the measured uncertainty in $d_{0}$, and by requiring that their longitudinal impact parameter, $z_{0}$, satisfies $\left|z_{0} \sin \theta\right|<0.5 \mathrm{~mm}$. To further reduce the background from non-prompt leptons, photon conversions and hadrons, lepton candidates are also required to be isolated. A lepton isolation criterion is defined by calculating the quantity $I_{R}=\sum p_{\mathrm{T}}^{\mathrm{trk}}$, where the sum includes all tracks (excluding the lepton candidate itself) within the cone defined by $\Delta R<R_{\text {cut }}$ about the direction of the lepton. The value of $R_{\text {cut }}$ is the smaller of $r_{\text {min }}$ and $10 \mathrm{GeV} / p_{\mathrm{T}}^{\ell}$, where $r_{\min }$ is set to 0.2 (0.3) for electron (muon) candidates, and $p_{\mathrm{T}}^{\ell}$ is the lepton $p_{\mathrm{T}}$. All lepton candidates must satisfy $I_{R} / p_{\mathrm{T}}^{\ell}<0.06$.

Candidate jets are reconstructed with the anti- $k_{t}$ algorithm [34-36] with a radius parameter $R=0.4$ (referred to as "small- $R$ jets"), using topological clusters [37] built from energy deposits in the calorimeters calibrated to the electromagnetic scale. The reconstructed jets are then calibrated to the particle level by the application of a jet energy scale derived from simulation and in situ corrections based on $\sqrt{s}=13 \mathrm{TeV}$ data [38]. Calibrated jets are required to have $p_{\mathrm{T}}>25 \mathrm{GeV}$ and $|\eta|<2.5$. Quality criteria are imposed to reject events that contain any jets arising from non-collision sources or detector noise [39]. To reduce the contamination due to jets originating from pile-up interactions, an additional requirement on the Jet Vertex Tagger (JVT) [40] output is made for jets with $p_{\mathrm{T}}<60 \mathrm{GeV}$ and $|\eta|<2.4$.

Jets containing $b$-hadrons are identified (b-tagged) via an algorithm $[41,42]$ that uses multivariate techniques to combine information about the impact parameters of displaced tracks and the topological properties of secondary and tertiary decay vertices reconstructed within the jet. For each jet, a value for the multivariate $b$-tagging discriminant is calculated. In this analysis, a jet is considered $b$-tagged if this value is above the threshold 
corresponding to an average $77 \%$ efficiency to tag a $b$-quark jet, with a light-jet ${ }^{2}$ rejection factor of $\sim 134$ and a charm-jet rejection factor of $\sim 6.2$, as determined for jets with $p_{\mathrm{T}}>20 \mathrm{GeV}$ and $|\eta|<2.5$ in simulated $t \bar{t}$ events.

Overlaps between candidate objects are removed sequentially. Firstly, electron candidates that lie within $\Delta R=0.01$ of a muon candidate are removed to suppress contributions from muon bremsstrahlung. Overlaps between electron and jet candidates are resolved next, and finally, overlaps between remaining jet candidates and muon candidates are removed. Clusters from identified electrons are not excluded during jet reconstruction. In order to avoid double-counting of electrons as jets, the closest jet whose axis is within $\Delta R=0.2$ of an electron is discarded. If the electron is within $\Delta R=0.4$ of the axis of any jet after this initial removal, the jet is retained and the electron is removed. The overlap removal procedure between the remaining jet candidates and muon candidates is designed to remove those muons that are likely to have arisen in the decay chain of hadrons and to retain the overlapping jet instead. Jets and muons may also appear in close proximity when the jet results from high- $p_{\mathrm{T}}$ muon bremsstrahlung, and in such cases the jet should be removed and the muon retained. Such jets are characterised by having very few matching inner-detector tracks. Selected muons that satisfy $\Delta R(\mu$, jet $)<0.04+10 \mathrm{GeV} / p_{\mathrm{T}}^{\mu}$ are rejected if the jet has at least three tracks originating from the primary vertex; otherwise the jet is removed and the muon is kept.

The candidate small- $R$ jets surviving the overlap removal procedure discussed above are used as inputs for further jet reclustering [43] using the anti- $k_{t}$ algorithm with a radius parameter $R=1.0$. In this way it is possible to evaluate the uncertainty in the mass of the large- $R$ jets that arises from the uncertainties in the energy scale and resolution of its constituent small- $R$ jets. In order to suppress contributions from pile-up and soft radiation, the reclustered large- $R$ (RCLR) jets are trimmed [44] by removing all small- $R$ (sub)jets within a RCLR jet that have $p_{\mathrm{T}}$ below $5 \%$ of the $p_{\mathrm{T}}$ of the reclustered jet. Due to the pile-up suppression and $p_{\mathrm{T}}>25 \mathrm{GeV}$ requirements made on the small- $R$ jets, the average fraction of small- $R$ jets removed by the trimming requirement is less than $1 \%$. The resulting RCLR jets are required to have $|\eta|<2.0$ and are used to identify high- $p_{\mathrm{T}}$ hadronically decaying top quark or Higgs boson candidates by making requirements on their transverse momentum, mass, and number of constituents. Hadronically decaying top quark candidates are reconstructed as RCLR jets with $p_{\mathrm{T}}>300 \mathrm{GeV}$, mass larger than $140 \mathrm{GeV}$, and at least two subjets. Higgs boson candidates are reconstructed as RCLR jets with $p_{\mathrm{T}}>200 \mathrm{GeV}$, a mass between 105 and $140 \mathrm{GeV}$, and a $p_{\mathrm{T}}$-dependent requirement on the number of subjets (exactly two for $p_{\mathrm{T}}<500 \mathrm{GeV}$, and one or two for $p_{\mathrm{T}}>500 \mathrm{GeV}$ ). In the following, these are referred to as "top-tagged" and "Higgs-tagged" jets, respectively, while the term "jet" without further qualifiers is used to refer to small- $R$ jets.

The missing transverse momentum $\vec{p}_{\mathrm{T}}^{\text {miss }}$ (with magnitude $E_{\mathrm{T}}^{\text {miss }}$ ) is defined as the negative vector sum of the $p_{\mathrm{T}}$ of all selected and calibrated objects in the event, including a term to account for energy from soft particles in the event which are not associated with any of the selected objects. This soft term is calculated from inner-detector tracks

\footnotetext{
${ }^{2}$ Light-jet refers to a jet originating from the hadronisation of a light quark $(u, d, s)$ or a gluon.
} 
matched to the selected primary vertex to make it more resilient to contamination from pile-up interactions $[45,46]$.

\section{Data sample and event preselection}

This search is based on a dataset of $p p$ collisions at $\sqrt{s}=13 \mathrm{TeV}$ with $25 \mathrm{~ns}$ bunch spacing collected by the ATLAS experiment in 2015 and 2016, corresponding to an integrated luminosity of $36.1 \mathrm{fb}^{-1}$. Only events recorded with a single-electron trigger, a singlemuon trigger, or a $E_{\mathrm{T}}^{\text {miss }}$ trigger under stable beam conditions and for which all detector subsystems were operational are considered.

Single-lepton triggers with low $p_{\mathrm{T}}$ threshold and lepton isolation requirements are combined in a logical OR with higher-threshold triggers without isolation requirements to give maximum efficiency. For muon triggers, the lowest $p_{\mathrm{T}}$ threshold is 20 (26) $\mathrm{GeV}$ in 2015 (2016), while the higher $p_{\mathrm{T}}$ threshold is $50 \mathrm{GeV}$ in both years. For electrons, triggers with a $p_{\mathrm{T}}$ threshold of 24 (26) $\mathrm{GeV}$ in 2015 (2016) and isolation requirements are used along with triggers with a $60 \mathrm{GeV}$ threshold and no isolation requirement, and with a 120 (140) $\mathrm{GeV}$ threshold with looser identification criteria. The $E_{\mathrm{T}}^{\text {miss }}$ trigger [29] considered uses an $E_{\mathrm{T}}^{\text {miss }}$ threshold of $70 \mathrm{GeV}$ in the HLT in 2015 and a run-period-dependent $E_{\mathrm{T}}^{\text {miss }}$ threshold varying between $90 \mathrm{GeV}$ and $110 \mathrm{GeV}$ in 2016 .

Events satisfying the trigger selection are required to have at least one primary vertex candidate. They are then classified into the "1-lepton" or "0-lepton" channels depending on the multiplicity of selected leptons. Events in the 1-lepton channel are required to satisfy a single-lepton trigger and to have exactly one selected electron or muon that matches, with $\Delta R<0.15$, the lepton reconstructed by the trigger. In the following, 1-lepton events satisfying either the electron or muon selections are combined and treated as a single analysis channel. Events in the 0-lepton channel are required to satisfy the $E_{\mathrm{T}}^{\text {miss }}$ trigger and to have no selected leptons. In addition, events in the 1-lepton (0-lepton) channel are required to have $\geq 5(\geq 6)$ small- $R$ jets. In the following, all selected small- $R$ jets are considered, including those used to build large- $R$ jets. For both channels, backgrounds that do not include $b$-quark jets are suppressed by requiring at least two $b$-tagged jets.

Additional requirements are made to suppress the background from multijet production. In the case of the 1-lepton channel, requirements are made on $E_{\mathrm{T}}^{\text {miss }}$ as well as on the transverse mass of the lepton and $E_{\mathrm{T}}^{\text {miss }}$ system $\left(m_{\mathrm{T}}^{W}\right):{ }^{3} E_{\mathrm{T}}^{\text {miss }}>20 \mathrm{GeV}$ and $E_{\mathrm{T}}^{\text {miss }}+m_{\mathrm{T}}^{W}>60 \mathrm{GeV}$. In the case of the 0-lepton channel, the requirements are $E_{\mathrm{T}}^{\text {miss }}>200 \mathrm{GeV}$ (for which the $E_{\mathrm{T}}^{\text {miss }}$ trigger is fully efficient) and $\Delta \phi_{\min }^{4 \mathrm{j}}>0.4$, where $\Delta \phi_{\min }^{4 \mathrm{j}}$ is the minimum azimuthal separation between $\vec{p}_{\mathrm{T}}^{\text {miss }}$ and each of the four highest- $p_{\mathrm{T}}$ jets. The latter requirement in the 0-lepton channel is very effective in suppressing multijet events, where the large $E_{\mathrm{T}}^{\text {miss }}$ results from the mismeasurement of a high- $p_{\mathrm{T}}$ jet or the presence of neutrinos emitted close to a jet axis.

\footnotetext{
${ }^{3} m_{\mathrm{T}}^{W}=\sqrt{2 p_{\mathrm{T}}^{\ell} E_{\mathrm{T}}^{\text {miss }}(1-\cos \Delta \phi)}$, where $p_{\mathrm{T}}^{\ell}$ is the transverse momentum (energy) of the muon (electron) and $\Delta \phi$ is the azimuthal angle separation between the lepton and the direction of the missing transverse momentum.
} 


\begin{tabular}{|l|c|c|}
\hline \multicolumn{3}{|c|}{ Preselection requirements } \\
\hline Requirement & 1-lepton channel & 0-lepton channel \\
\hline Trigger & Single-lepton trigger & $E_{\mathrm{T}}^{\text {miss }}$ trigger \\
Leptons & $=1$ isolated $e$ or $\mu$ & $=0$ isolated $e$ or $\mu$ \\
Jets & $\geq 5$ jets & $\geq 6$ jets \\
$b$-tagging & $\geq 2 b$-tagged jets & $\geq 2$-tagged jets \\
$E_{\mathrm{T}}^{\text {miss }}$ & $E_{\mathrm{T}}^{\text {miss }}>20 \mathrm{GeV}$ & $E_{\mathrm{T}}^{\text {miss }}>200 \mathrm{GeV}$ \\
Other $E_{\mathrm{T}}^{\text {miss }}$-related & $E_{\mathrm{T}}^{\text {miss }}+m_{\mathrm{T}}^{W}>60 \mathrm{GeV}$ & $\Delta \phi_{\text {min }}^{4 \mathrm{j}}>0.4$ \\
\hline
\end{tabular}

Table 1. Summary of preselection requirements for the 1-lepton and 0-lepton channels. Here $m_{\mathrm{T}}^{W}$ is the transverse mass of the lepton and the $E_{\mathrm{T}}^{\mathrm{miss}}$ vector, and $\Delta \phi_{\min }^{4 \mathrm{j}}$ is the minimum azimuthal separation between the $E_{\mathrm{T}}^{\text {miss }}$ vector and each of the four highest- $p_{\mathrm{T}}$ jets.

The above requirements are referred to as the "preselection" and are summarised in table 1.

\section{$5 \quad$ Signal and background modelling}

Signal and most background processes were modelled using Monte Carlo (MC) simulations. In the simulation, the top quark and SM Higgs boson masses were set to $172.5 \mathrm{GeV}$ and $125 \mathrm{GeV}$, respectively. All simulated samples, except those produced with the SHERPA [47] event generator, utilised EvTGEN v1.2.0 [48] to model the decays of heavy-flavour hadrons. To model the effects of pile-up, events from minimum-bias interactions were generated using the Pythia 8.186 [49] event generator and overlaid onto the simulated hard-scatter events according to the luminosity profile of the recorded data. The generated events were processed through a simulation [50] of the ATLAS detector geometry and response using Geant4 [51]. A faster simulation, where the full Geant4 simulation of the calorimeter response is replaced by a detailed parameterisation of the shower shapes [52], was adopted for some of the samples used to estimate systematic uncertainties. Simulated events are processed through the same reconstruction software as the data, and corrections are applied so that the object identification efficiencies, energy scales and energy resolutions match those determined from data control samples.

\section{$5.1 \quad$ Signal modelling}

Samples of simulated $T \bar{T}$ events were generated with the leading-order (LO) generator ${ }^{4}$ Protos $2.2[8,53]$ using the NNPDF2.3 LO [54] parton distribution function (PDF) set and passed to Pyтнia 8.186 [49] for parton showering and fragmentation. The A14 [55] set of optimised parameters for the underlying event (UE) description using the NNPDF2.3 LO PDF set, referred to as the "A14 UE tune", was used. The samples were generated

\footnotetext{
${ }^{4}$ In the following, the order of a generator should be understood as referring to the order in the strong coupling constant at which the matrix element calculation is performed.
} 
assuming singlet couplings and for heavy-quark masses between $350 \mathrm{GeV}$ and $1.5 \mathrm{TeV}$ in steps of $50 \mathrm{GeV}$. Additional samples were produced at three mass points $(700 \mathrm{GeV}, 950 \mathrm{GeV}$ and $1.2 \mathrm{TeV}$ ) assuming doublet couplings in order to confirm that, at fixed branching fraction, kinematic differences arising from the different chirality of singlet and doublet couplings have negligible impact on this search. The vector-like quarks were forced to decay with a branching ratio of $1 / 3$ into each of the three modes $(W, Z, H)$. These samples were reweighted using generator-level information to allow results to be interpreted for arbitrary sets of branching ratios that are consistent with the three decay modes summing to unity. The generated samples were normalised to the theoretical cross sections computed using TOP ++ v2.0 [56] at next-to-next-to-leading order (NNLO) in quantum chromodynamics (QCD), including resummation of next-to-next-to-leading logarithmic (NNLL) soft gluon terms [57-61], and using the MSTW 2008 NNLO [62, 63] set of PDFs. The predicted pair-production cross section at $\sqrt{s}=13 \mathrm{TeV}$ ranges from $24 \mathrm{pb}$ for a vector-like quark mass of $350 \mathrm{GeV}$ to $2.0 \mathrm{fb}$ for a mass of $1.5 \mathrm{TeV}$, with an uncertainty that increases from $8 \%$ to $18 \%$ over this mass range. The theoretical uncertainties result from variations of the factorisation and renormalisation scales, as well as from uncertainties in the PDF and $\alpha_{\mathrm{S}}$. The latter two represent the largest contribution to the overall theoretical uncertainty in the cross section and were calculated using the PDF4LHC prescription [64] with the MSTW 2008 68\% CL NNLO, CT10 NNLO [65, 66] and NNPDF2.3 5f FFN [54] PDF sets.

Samples of simulated four-top-quark events produced via an EFT and within the 2UED/RPP model were generated at LO with the MADGRAPH5_aMC@NLO [67] generator (referred to in the following as MG5_aMC; the versions used are 2.2.3 and 1.5.14 for EFT and 2UED/RPP, respectively) and the NNPDF2.3 LO PDF set, interfaced to PYTHIA 8 (the versions used are 8.205 and 8.186 for EFT and 2UED/RPP, respectively) and the A14 UE tune. The EFT $t \bar{t} t \bar{t}$ sample was normalised assuming $\left|C_{4 t}\right| / \Lambda^{2}=4 \pi \mathrm{TeV}^{-2}$, where $C_{4 t}$ denotes the coupling constant and $\Lambda$ the energy scale of new physics, which yields a cross section of $928 \mathrm{fb}$ computed using MG5_aMC. In the case of the 2UED/RPP model, samples were generated for four different values of $m_{\mathrm{KK}}$ (from $1 \mathrm{TeV}$ to $1.8 \mathrm{TeV}$ in steps of $200 \mathrm{GeV}$ ) and the BRIDGE [68] generator was used to decay the pair-produced excitations from tier $(1,1)$ generated by MADGRAPH5. The corresponding predicted cross section ranges from $343 \mathrm{fb}$ for $m_{\mathrm{KK}}=1 \mathrm{TeV}$ to $1.1 \mathrm{fb}$ for $m_{\mathrm{KK}}=1.8 \mathrm{TeV}$.

\subsection{Background modelling}

After the event preselection, the main background is $t \bar{t}$ production, often in association with jets, denoted by $t \bar{t}+$ jets in the following. Small contributions arise from single-topquark, $W / Z+$ jets, multijet and diboson $(W W, W Z, Z Z)$ production, as well as from the associated production of a vector boson $V(V=W, Z)$ or a Higgs boson and a $t \bar{t}$ pair $(t \bar{t} V$ and $t \bar{t} H)$. All backgrounds are estimated using samples of simulated events and initially normalised to their theoretical cross sections, with the exception of the multijet background, which is estimated using data-driven methods. The background prediction is further improved during the statistical analysis by performing a likelihood fit to data using multiple signal-depleted search regions, as discussed in section 6 . 
The nominal sample used to model the $t \bar{t}$ background was generated with the NLO generator Powheg-Box v2 [69-72] using the CT10 PDF set [65]. The Powheg-Box model parameter $h_{\text {damp }}$, which controls matrix element to parton shower matching and effectively regulates the high- $p_{\mathrm{T}}$ radiation, was set to the top quark mass, a setting that was found to describe the $t \bar{t}$ system's $p_{\mathrm{T}}$ at $\sqrt{s}=7 \mathrm{TeV}$ [73]. The nominal $t \bar{t}$ sample was interfaced to Pythia 6.428 [74] with the CTEQ6L PDF set and the Perugia 2012 (P2012) UE tune [75]. Alternative $t \bar{t}$ simulation samples used to derive systematic uncertainties are described in section 7.3.

All $t \bar{t}$ samples were generated inclusively, but events are categorised depending on the flavour content of additional particle jets not originating from the decay of the $t \bar{t}$ system (see ref. [76] for details). Events labelled as either $t \bar{t}+\geq 1 b$ or $t \bar{t}+\geq 1 c$ are generically referred in the following as $t \bar{t}+\mathrm{HF}$ events, where HF stands for "heavy flavour". A finer categorisation of $t \bar{t}+\geq 1 b$ events is considered for the purpose of applying further corrections and assigning systematic uncertainties associated with the modelling of heavy-flavour production in different topologies [76]. The remaining events are labelled as $t \bar{t}+$ light-jets events, including those with no additional jets. In previous studies, better agreement between data and prediction was observed, particularly for the top quark $p_{\mathrm{T}}$ distribution, when comparing to NNLO calculations [77]. These small improvements to the modelling are incorporated by reweighting all $t \bar{t}$ samples to match their top quark $p_{\mathrm{T}}$ distribution to that predicted at NNLO accuracy in QCD [78, 79]. This correction is not applied to $t \bar{t}+\geq 1 b$ events, which instead are reweighted to an NLO prediction in the four-flavour (4F) scheme of $t \bar{t}+\geq 1 b$ including parton showering [80], based on SHERPA+OpenLoops [47, 81] (referred to as SherPaOL in the following) using the CT10 PDF set. This reweighting is performed separately for each of the $t \bar{t}+\geq 1 b$ categories in such a way that their inter-normalisation and the shape of the relevant kinematic distributions are at NLO accuracy, while preserving the nominal $t \bar{t}+\geq 1 b$ cross section in Powheg-Box + Pythia. The corrections described in this paragraph are applied to the nominal as well as the alternative $t \bar{t}$ samples.

Samples of single-top-quark events corresponding to the $t$-channel production mechanism were generated with the Powheg-Box v1 [82] generator that uses the $4 \mathrm{~F}$ scheme for the NLO matrix element calculations and the fixed 4 F CT10f4 [65] PDF set. Samples corresponding to the $W t$ - and $s$-channel production mechanisms were generated with Powheg-Box v2 using the CT10 PDF set. Overlaps between the $t \bar{t}$ and $W t$ final states are avoided by using the "diagram removal" scheme [83]. The parton shower, hadronisation and the underlying event are modelled using PYTHIA 6.428 with the CTEQ6L1 PDF set in combination with the P2012 UE tune. The single-top-quark samples were normalised to the approximate NNLO theoretical cross sections [84-86].

Samples of $W / Z+$ jets events were generated with the SHERPA 2.2 [47] generator. The matrix element was calculated for up to two partons at NLO and up to four partons at LO using Comix [87] and OpenLoops [81]. The matrix element calculation was merged with the SHERPA parton shower [88] using the ME+PS@NLO prescription [89]. The PDF set used for the matrix-element calculation is NNPDF3.0NNLO [90] with a dedicated parton shower tuning developed for SHERPA. Separate samples were generated for different $W / Z+$ jets categories using filters for a $b$-jet $(W / Z+\geq 1 b+$ jets $)$, a $c$-jet and no $b$-jet 
$(W / Z+\geq 1 c+$ jets $)$, and with a veto on $b$ - and $c$-jets ( $W / Z+$ light-jets), which were combined into the inclusive $W / Z+$ jets samples. Both the $W+$ jets and $Z+$ jets samples were normalised to their respective inclusive NNLO theoretical cross sections in QCD calculated with FEWZ [91].

Samples of $W W / W Z / Z Z+$ jets events were generated with SHERPA 2.1.1 using the CT10 PDF set and include processes containing up to four electroweak vertices. The matrix element includes zero additional partons at NLO and up to three partons at LO using the same procedure as for the $W / Z+$ jets samples. The final states simulated require one of the bosons to decay leptonically and the other hadronically. All diboson samples were normalised to their NLO theoretical cross sections provided by SHERPA.

Samples of $t \bar{t} V$ and $t \bar{t} H$ events were generated with MG5_aMC 2.3.2, using NLO matrix elements and the NNPDF3.0NLO [90] PDF set. Showering was performed using Pythia 8.210 and the A14 UE tune. The $t \bar{t} V$ samples were normalised to the NLO cross section computed with MG5_aMC. The $t \bar{t} H$ sample was normalised using the NLO cross section [92-96] and the Higgs boson decay branching ratios calculated using HDECAY [97].

The production of four-top-quark events in the SM was simulated by samples generated at LO using MG5_aMC 2.2.2 and the NNPDF2.3 LO PDF set, interfaced to PyтнIA 8.186 in combination with the A14 UE tune. The sample was normalised to a cross section of $9.2 \mathrm{fb}$, computed at NLO [67].

The background from multijet production ("multijet background" in the following) in the 1-lepton channel contributes to the selected data sample via several production and misreconstruction mechanisms. In the electron channel, it consists of non-prompt electrons (from semileptonic $b$ - or $c$-hadron decays) as well as misidentified photons (e.g. from a conversion of a photon into an $e^{+} e^{-}$pair) or jets with a high fraction of their energy deposited in the EM calorimeter. In the muon channel, the multijet background is predominantly from non-prompt muons. The multijet background normalisation and shape are estimated directly from data by using the "matrix method" technique [98], which exploits differences in lepton identification and isolation properties between prompt leptons and leptons that are either non-prompt or result from the misidentification of photons or jets. Further details can be found in ref. [25]. The main type of multijet background that contributes to the 0-lepton channel are events in which the energy of a high- $p_{\mathrm{T}}$ jet is mismeasured, consequently leading to a large missing transverse momentum in the final state. Most of this background is suppressed by selecting events satisfying $\Delta \phi_{\min }^{4 \mathrm{j}}>0.4$. The remaining multijet background in each search region is estimated from a control region defined with the same selection as the search region, but with the selection on $\Delta \phi_{\text {min }}^{4 \mathrm{j}}$ changed to $\Delta \phi_{\min }^{4 \mathrm{j}}<0.1$. The normalisation of the multijet background is extrapolated from the control region to its corresponding search region by performing an exponential fit to the $\Delta \phi_{\min }^{4 \mathrm{j}}$ distribution in the range $0<\Delta \phi_{\min }^{4 \mathrm{j}}<0.4$. The background prediction is validated by comparing the data and total prediction in multijet-rich samples selected by choosing ranges of $\Delta \phi_{\min }^{4 \mathrm{j}}$ (e.g. $0.3<\Delta \phi_{\min }^{4 \mathrm{j}}<0.4$ ). 


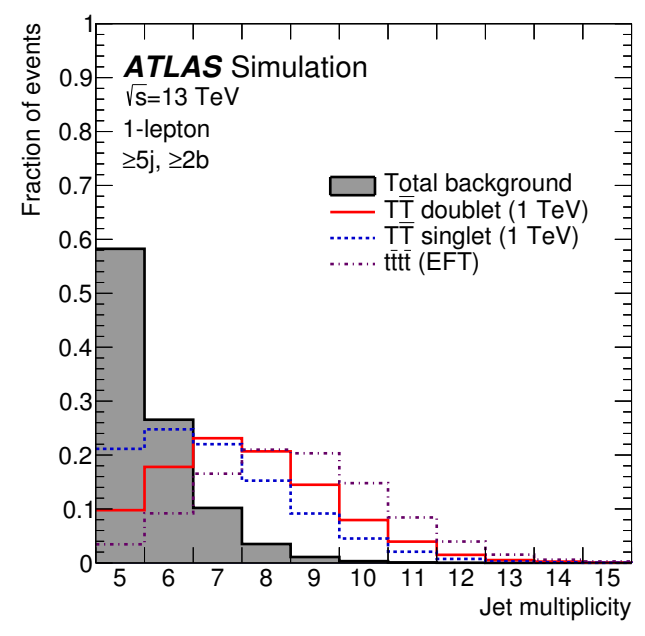

(a)

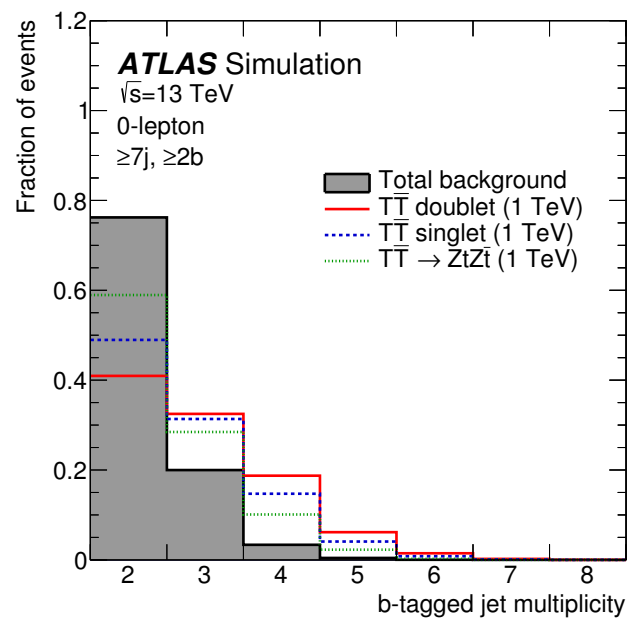

(b)

Figure 2. Comparison of the distribution of (a) the jet multiplicity, and (b) the $b$-tagged jet multiplicity, between the total background (shaded histogram) and several signal scenarios considered in this search. The selection used in (a) corresponds to events in the 1-lepton channel satisfying the preselection requirements, whereas the selection used in (b) corresponds to events in the 0-lepton channel satisfying the preselection requirements and $\geq 7$ jets. The signals shown correspond to: $T \bar{T}$ production in the weak-isospin doublet and singlet scenarios, and in the $\mathcal{B}(T \rightarrow Z t)=1$ case, assuming $m_{T}=1 \mathrm{TeV}$; and $t \bar{t} t \bar{t}$ production within an EFT model.

\section{$6 \quad$ Search strategy}

The searches discussed in this paper primarily target $T \bar{T}$ production where at least one of the $T$ quarks decays into a Higgs boson and a top quark resulting in the following processes: $T \bar{T} \rightarrow H t H t, H t Z t$ and $H t W b{ }^{5}$ For the dominant $H \rightarrow b \bar{b}$ decay mode, the final-state signatures in both the 1-lepton and 0-lepton searches are characterised by high jet and $b$-tagged jet multiplicities, which provide a powerful experimental handle to suppress the background. The presence of high-momentum $Z$ bosons decaying into $\nu \bar{\nu}$ or $W$ bosons decaying leptonically, either to an electron or muon that is not reconstructed or to a hadronically decaying $\tau$-lepton that is identified as a jet, yields high $E_{\mathrm{T}}^{\text {miss }}$, which is exploited by the 0-lepton search. Both searches have some sensitivity to $T \bar{T} \rightarrow Z t Z t$ and $Z t W b$, with $Z \rightarrow b \bar{b}$. Possible contributions from pair production of the $B$ or $X$ quarks that would be included, along with the $T$ quark, in a weak-isospin doublet are ignored. Such particles are expected to decay primarily through $X, B \rightarrow W t[8]$, and thus not lead to high $b$-tagged jet multiplicity, which is the primary focus of these searches. High jet and $b$-tagged jet multiplicities are also characteristic of $t \bar{t} t \bar{t}$ events (both within the SM and in BSM scenarios); this search is sensitive to these events. The four-top-quark production scenarios considered here do not feature large $E_{\mathrm{T}}^{\text {miss }}$, so only the 1-lepton search is used to probe them. No dedicated re-optimisation for $t \bar{t} t \bar{t}$ events was performed.

\footnotetext{
${ }^{5}$ In the following, $H t Z t$ is used to denote both $H t Z \bar{t}$ and its charge conjugate, $H \bar{t} Z t$. Similar notation is used for other processes, as appropriate.
} 


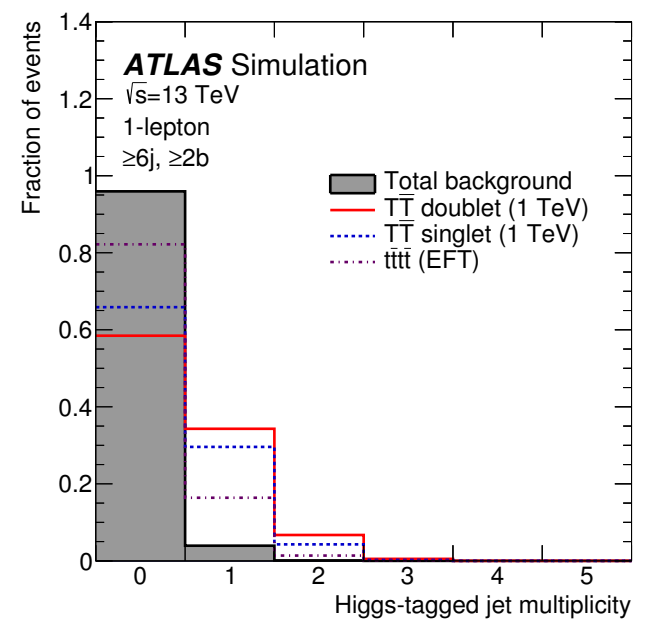

(a)

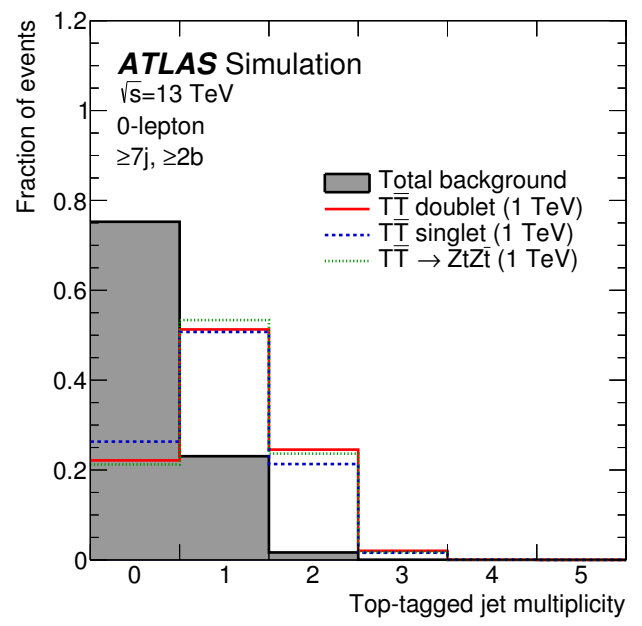

(b)

Figure 3. Comparison of the distribution of (a) the Higgs-tagged jet multiplicity and (b) the top-tagged jet multiplicity, between the total background (shaded histogram) and several signal scenarios considered in this search. The selection used in (a) corresponds to events in the 1-lepton channel satisfying the preselection requirements and $\geq 6$ jets, whereas the selection used in (b) corresponds to events in the 0-lepton channel satisfying the preselection requirements and $\geq 7$ jets. The signals shown correspond to: $T \bar{T}$ production in the weak-isospin doublet and singlet scenarios, and in the $\mathcal{B}(T \rightarrow Z t)=1$ case, assuming $m_{T}=1 \mathrm{TeV}$; and $t \bar{t} t \bar{t}$ production within an EFT model.

In figure 2(a) the jet multiplicity distribution in the 1-lepton channel after preselection (described in section 4) is compared between the total background and several signal scenarios, chosen to illustrate differences among various types of signals the search is sensitive to. A similar comparison for the $b$-tagged jet multiplicity distribution is shown in figure 2 (b) for events in the 0-lepton channel after preselection plus the requirement of $\geq 7$ jets.

Compared to Run 1, the larger centre-of-mass energy in Run 2 provides sensitivity to higher-mass signals, which decay into boosted heavy SM particles (particularly Higgs bosons and top quarks). These potentially give rise to a high multiplicity of large- $R$ jets that capture their decay products (see section 3 ). While $t \bar{t}+$ jets events in the 1-lepton and 0-lepton channels are expected to typically contain one top-tagged jet, the signal events of interest are characterised by higher Higgs-tagged jet and top-tagged jet multiplicities, as illustrated in figures 3(a) and 3(b). The small fraction (about 5\%) of background events with $\geq 2$ top-tagged jets or $\geq 1$ Higgs-tagged jets results from the misidentification of at least one large- $R$ jet where initial- or final-state radiation was responsible for a large fraction of the constituents.

In order to optimise the sensitivity of the searches, the selected events are categorised into different regions depending on the jet multiplicity $(5$ and $\geq 6$ jets in the 1-lepton channel; 6 and $\geq 7$ jets in the 0-lepton channel), $b$-tagged jet multiplicity ( 3 and $\geq 4$ in the 1-lepton channel; 2, 3 and $\geq 4$ in the 0-lepton channel) and Higgs- and top-tagged jet 


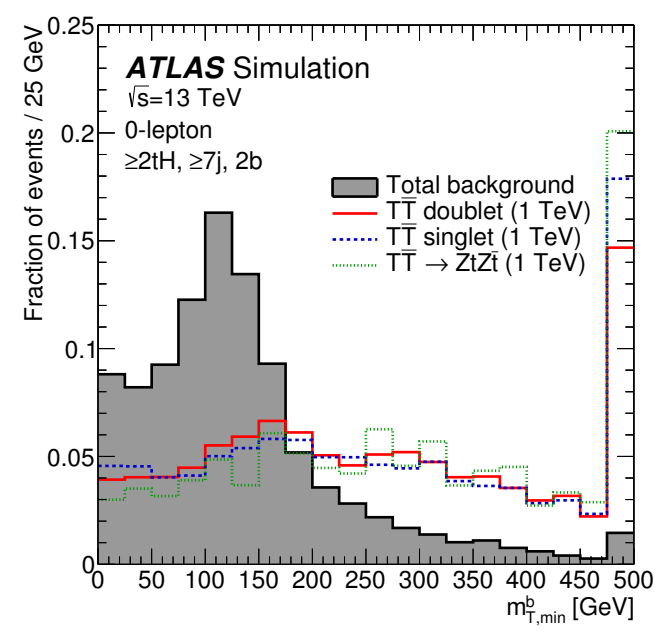

Figure 4. Comparison of the distribution of the minimum transverse mass of $E_{\mathrm{T}}^{\text {miss }}$ and any of the three (or two, in events with exactly two $b$-tagged jets) leading $b$-tagged jets in the event $\left(m_{\mathrm{T}, \min }^{\mathrm{b}}\right)$, between the total background (shaded histogram) and several signal scenarios considered in this search. The selection used corresponds to events in the $(\geq 2 \mathrm{tH}, \geq 7 \mathrm{j}, 2 \mathrm{~b})$ region of the 0-lepton channel. The signals shown correspond to $T \bar{T}$ production in the weak-isospin doublet and singlet scenarios, and in the $\mathcal{B}(T \rightarrow Z t)=1$ case, assuming $m_{T}=1 \mathrm{TeV}$. The last bin in the figure contains the overflow.

multiplicity $(0,1$ and $\geq 2)$. In the following, channels with $N_{\mathrm{t}}$ top-tagged jets, $N_{\mathrm{H}}$ Higgstagged jets, $n$ jets, and $m b$-tagged jets are denoted by " $N_{\mathrm{t}} \mathrm{t}, N_{\mathrm{H}} \mathrm{H}, n \mathrm{j}, m \mathrm{~b}$ ". Whenever the top/Higgs-tagging requirement is made on the sum $N_{\mathrm{t}}+N_{\mathrm{H}} \equiv N_{\mathrm{tH}}$, the channel is denoted by " $N_{\mathrm{tH}} \mathrm{tH}, n \mathrm{j}, m \mathrm{~b}$ ". In addition, events in the 0 -lepton channel are further categorised into two regions according to the value of $m_{\mathrm{T}, \text { min }}^{\mathrm{b}}$, the minimum transverse mass of $E_{\mathrm{T}}^{\text {miss }}$ and any of the three (or two, in events with exactly two $b$-tagged jets) leading $b$-tagged jets in the event: $m_{\mathrm{T}, \text { min }}^{\mathrm{b}}<160 \mathrm{GeV}$ (referred to as "LM", standing for "low mass") and $m_{\mathrm{T}, \text { min }}^{\mathrm{b}}>160 \mathrm{GeV}$ (referred to as "HM", standing for "high mass"). This kinematic variable is bounded from above by the top quark mass for semileptonic $t \bar{t}$ background events, while the signal can have higher values of $m_{\mathrm{T}, \text { min }}^{\mathrm{b}}$ due to the presence of high- $p_{\mathrm{T}}$ neutrinos from $T \rightarrow Z t, Z \rightarrow \nu \nu$ or $T \rightarrow W b, W \rightarrow \ell \nu$ decays. Although the requirements of a minimum top/Higgs-tagged jet multiplicity reduces the value of $m_{\mathrm{T}}^{\mathrm{b}}$, min because of the resulting stronger collimation of the top quark decay products, this variable still provides useful discrimination between signal and $t \bar{t}$ background, as shown in figure 4 . While the 1-lepton channel only considers regions with exactly 3 or $\geq 4$ b-tagged jets, the 0 -lepton channel also includes regions with exactly two $b$-jets and $m_{\mathrm{T} \text {, min }}^{\mathrm{b}}>160 \mathrm{GeV}$, to gain sensitivity to $T \bar{T} \rightarrow Z t Z t$ decays with at least one $Z \rightarrow \nu \bar{\nu}$ decay.

To further improve the separation between the $T \bar{T}$ signal and background, the distinct kinematic features of the signal are exploited. In particular, the large $T$ quark mass results in leptons and jets with large energy in the final state and the effective mass $\left(m_{\mathrm{eff}}\right)$, defined as the scalar sum of the transverse momenta of the lepton, the selected jets and the missing transverse momentum, provides a powerful discriminating variable between signal 
and background. The $m_{\text {eff }}$ distribution peaks at approximately $2 m_{T}$ for signal events and at lower values for the $t \bar{t}+$ jets background. For the same reasons, the various $t \bar{t} t \bar{t}$ signals from BSM scenarios also populate high values of $m_{\mathrm{eff}}$. An additional selection requirement of $m_{\mathrm{eff}}>1 \mathrm{TeV}$ is made in order to minimise the effect of possible mismodelling of the $m_{\text {eff }}$ distribution at low values originating from small backgrounds with large systematic uncertainties, such as multijet production. Such a requirement is applied for regions with $N_{\mathrm{t}}+N_{\mathrm{H}} \leq 1$ in the 1-lepton channel, and for all regions in the 0-lepton channel. Since the $T \bar{T}$ signal is characterised by having at least one top/Higgs-tagged jet and large values of $m_{\text {eff }}$, this minimum requirement on $m_{\text {eff }}$ does not decrease the signal efficiency. In figure 5 , the $m_{\text {eff }}$ distribution is compared between signal and background for events in signal-rich regions of the 1-lepton and 0-lepton channels. The kinematic requirements in these regions result in a significantly harder $m_{\text {eff }}$ spectrum for the background than in regions without top/Higgs-tagged jets, but this variable still shows good discrimination between signal and background. Thus, the $m_{\text {eff }}$ distribution is used as the final discriminating variable in all regions considered in this search.

The regions with $\geq 6$ jets ( $\geq 7$ jets) are used to perform the search in the 1-lepton ( 0 lepton) channel (referred to as "search regions"), whereas the regions with exactly 5 jets ( 6 jets) are used to validate the background modelling in different regimes of event kinematics and heavy-flavour content (referred to as "validation regions"). A total of 12 search regions and 10 validation regions are considered in the 1-lepton channel, whereas 22 search regions and 16 validation regions are considered in the 0-lepton channel, defined in tables 2 and 3 respectively. In each channel, there are fewer validation regions than signal regions since some validation regions are merged to ensure a minimum of about 10 expected events. The level of possible signal contamination in the validation regions that have high event yields, and are therefore the regions that are most useful to validate the background prediction, depends on the signal scenario considered but is typically well below $10 \%$ for a $1 \mathrm{TeV} T$ quark.

The overall rate and composition of the $t \bar{t}+$ jets background strongly depends on the jet and $b$-tagged jet multiplicities, as illustrated in figure 6 . The $t \bar{t}+$ light-jets background is dominant in events with exactly two $b$-tagged jets, which typically correspond to the two $b$-quarks from the top quark decays. It also contributes significantly to events with exactly three $b$-tagged jets, in which typically a charm quark from the hadronic $W$ boson decay is also $b$-tagged. Contributions from $t \bar{t}+\geq 1 c$ and $t \bar{t}+\geq 1 b$ become significant as the $b$-tagged jet multiplicity increases, with the $t \bar{t}+\geq 1 b$ background being dominant for events with $\geq 4 b$-tagged jets. The regions with different top/Higgs-tagged jet multiplicities probe different kinematic regimes, both soft (e.g. low-mass $T$ quark) and hard (e.g. high-mass $T$ quark or BSM $t \bar{t} t \bar{t}$ production). The search regions with the higher multiplicities of top/Higgs-tagged jets and $b$-tagged jets in both the 1-lepton and 0-lepton channels, as well as the HM regions in the 0-lepton channel, have the largest signal-to-background ratio, and therefore drive the sensitivity of the search. The remaining search regions have significantly lower signal-to-background ratios, but are useful for checking and correcting the $t \bar{t}+$ jets background prediction and constraining the related systematic uncertainties (see section 7) through a likelihood fit to data (see section 8). A summary of the signal-to-background ratio in the different search regions is displayed in figure 7 for the $T$ quark signal with 


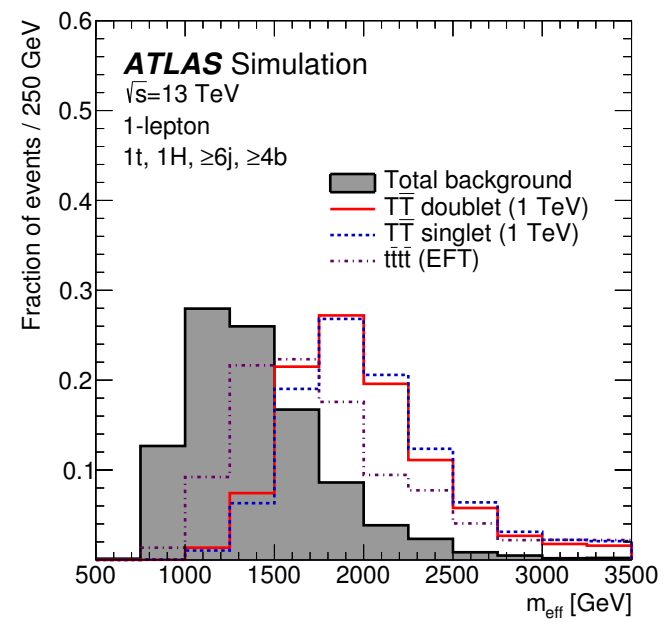

(a)

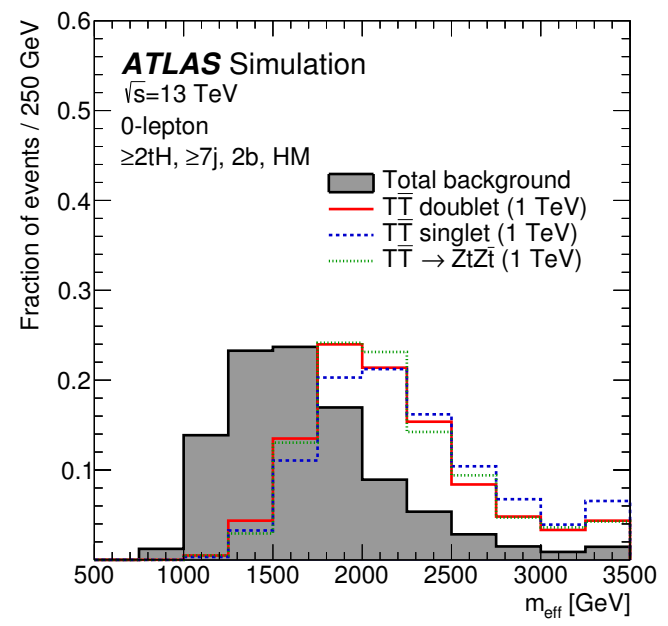

(b)

Figure 5. Comparison of the distribution of the effective mass $\left(m_{\mathrm{eff}}\right)$, between the total background (shaded histogram) and several signal scenarios considered in this search. The selection used in (a) corresponds to events in the $(1 \mathrm{t}, 1 \mathrm{H}, \geq 6 \mathrm{j}, \geq 4 \mathrm{~b})$ region of the 1-lepton channel, whereas the selection used in (b) corresponds to events in the ( $\geq 2 \mathrm{tH}, \geq 7 \mathrm{j}, 2 \mathrm{~b}, \mathrm{HM})$ region of the 0-lepton channel. The signals shown correspond to: $T \bar{T}$ production in the weak-isospin doublet and singlet scenarios, and in the $\mathcal{B}(T \rightarrow Z t)=1$ case, assuming $m_{T}=1 \mathrm{TeV}$; and $t \bar{t} t \bar{t}$ production within an EFT model. The last bin in each distribution contains the overflow.

various decay configurations. A similar fitting strategy was followed in the Run-1 search in the 1-lepton channel [25].

A summary of the observed and expected yields before the fit to data in five of the most sensitive search regions in the 1-lepton and 0-lepton channels can be found in tables 4 and 5, respectively. The search regions shown in table 4 for the 1-lepton channel are a selection of some of the regions with the highest $S / \sqrt{B}$ ratio (where $S$ and $B$ are the expected signal and background yields, respectively) across several signal benchmark scenarios considered $(T \bar{T}$ in the $\mathcal{B}(T \rightarrow H t)=1, T$ doublet, and $T$ singlet scenarios, in all cases assuming $m_{T}=1 \mathrm{TeV}$, and $t \bar{t} t \bar{t}$ within an EFT and the 2UED/RPP models). Similarly, the search regions shown in table 5 for the 0-lepton channel are a superset of the regions with the highest $S / \sqrt{B}$ ratio for different $T \bar{T}$ signal benchmark scenarios ( $T$ doublet, $T$ singlet and $\mathcal{B}(T \rightarrow Z t)=1$, also assuming $\left.m_{T}=1 \mathrm{TeV}\right)$.

\section{Systematic uncertainties}

Several sources of systematic uncertainty are considered that affect the normalisation of signal and background and/or the shape of their $m_{\text {eff }}$ distributions. Each source of systematic uncertainty is considered to be uncorrelated with the other sources. Correlations for a given systematic uncertainty are maintained across processes and channels, unless explicitly stated otherwise. 


\begin{tabular}{|c|c|c|c|c|}
\hline \multicolumn{5}{|c|}{ 1-lepton channel } \\
\hline \multicolumn{5}{|c|}{ Search regions ( $\geq 6$ jets $)$} \\
\hline$N_{\mathrm{t}}$ & $N_{\mathrm{H}}$ & $b$-tag multiplicity & $m_{\mathrm{eff}}$ & Channel name \\
\hline 0 & 0 & 3 & $>1 \mathrm{TeV}$ & $0 \mathrm{t}, 0 \mathrm{H}, \geq 6 \mathrm{j}, 3 \mathrm{~b}$ \\
\hline 0 & 0 & $\geq 4$ & $>1 \mathrm{TeV}$ & $0 \mathrm{t}, 0 \mathrm{H}, \geq 6 \mathrm{j}, \geq 4 \mathrm{~b}$ \\
\hline 1 & 0 & 3 & $>1 \mathrm{TeV}$ & $1 \mathrm{t}, 0 \mathrm{H}, \geq 6 \mathrm{j}, 3 \mathrm{~b}$ \\
\hline 1 & 0 & $\geq 4$ & $>1 \mathrm{TeV}$ & $1 \mathrm{t}, 0 \mathrm{H}, \geq 6 \mathrm{j}, \geq 4 \mathrm{~b}$ \\
\hline 0 & 1 & 3 & $>1 \mathrm{TeV}$ & $0 \mathrm{t}, 1 \mathrm{H}, \geq 6 \mathrm{j}, 3 \mathrm{~b}$ \\
\hline 0 & 1 & $\geq 4$ & $>1 \mathrm{TeV}$ & $0 \mathrm{t}, 1 \mathrm{H}, \geq 6 \mathrm{j}, \geq 4 \mathrm{~b}$ \\
\hline 1 & 1 & 3 & - & $1 \mathrm{t}, 1 \mathrm{H}, \geq 6 \mathrm{j}, 3 \mathrm{~b}$ \\
\hline 1 & 1 & $\geq 4$ & - & $1 \mathrm{t}, 1 \mathrm{H}, \geq 6 \mathrm{j}, \geq 4 \mathrm{~b}$ \\
\hline$\geq 2$ & 0 or 1 & 3 & - & $\geq 2 \mathrm{t}, 0-1 \mathrm{H}, \geq 6 \mathrm{j}, 3 \mathrm{~b}$ \\
\hline$\geq 2$ & 0 or 1 & $\geq 4$ & - & $\geq 2 \mathrm{t}, 0-1 \mathrm{H}, \geq 6 \mathrm{j}, \geq 4 \mathrm{~b}$ \\
\hline$\geq 0$ & $\geq 2$ & 3 & - & $\geq 0 \mathrm{t}, \geq 2 \mathrm{H}, \geq 6 \mathrm{j}, 3 \mathrm{~b}$ \\
\hline$\geq 0$ & $\geq 2$ & $\geq 4$ & - & $\geq 0 \mathrm{t}, \geq 2 \mathrm{H}, \geq 6 \mathrm{j}, \geq 4 \mathrm{~b}$ \\
\hline \multicolumn{5}{|c|}{ Validation regions ( 5 jets) } \\
\hline$N_{\mathrm{t}}$ & $N_{\mathrm{H}}$ & $b$-tag multiplicity & $m_{\mathrm{eff}}$ & Channel name \\
\hline 0 & 0 & 3 & $>1 \mathrm{TeV}$ & $0 \mathrm{t}, 0 \mathrm{H}, 5 \mathrm{j}, 3 \mathrm{~b}$ \\
\hline 0 & 0 & $\geq 4$ & $>1 \mathrm{TeV}$ & $0 \mathrm{t}, 0 \mathrm{H}, 5 \mathrm{j}, \geq 4 \mathrm{~b}$ \\
\hline 1 & 0 & 3 & $>1 \mathrm{TeV}$ & $1 \mathrm{t}, 0 \mathrm{H}, 5 \mathrm{j}, 3 \mathrm{~b}$ \\
\hline 1 & 0 & $\geq 4$ & $>1 \mathrm{TeV}$ & $1 \mathrm{t}, 0 \mathrm{H}, 5 \mathrm{j}, \geq 4 \mathrm{~b}$ \\
\hline 0 & 1 & 3 & $>1 \mathrm{TeV}$ & $0 \mathrm{t}, 1 \mathrm{H}, 5 \mathrm{j}, 3 \mathrm{~b}$ \\
\hline 0 & 1 & $\geq 4$ & $>1 \mathrm{TeV}$ & $0 \mathrm{t}, 1 \mathrm{H}, 5 \mathrm{j}, \geq 4 \mathrm{~b}$ \\
\hline 1 & 1 & 3 & - & $1 \mathrm{t}, 1 \mathrm{H}, 5 \mathrm{j}, 3 \mathrm{~b}$ \\
\hline$\geq 2$ & 0 or 1 & 3 & - & $\geq 2 \mathrm{t}, 0-1 \mathrm{H}, 5 \mathrm{j}, 3 \mathrm{~b}$ \\
\hline$\geq 0$ & $\geq 2$ & 3 & - & $\geq 0 \mathrm{t}, \geq 2 \mathrm{H}, 5 \mathrm{j}, 3 \mathrm{~b}$ \\
\hline$N_{\mathrm{t}}$ & $N_{\mathrm{H}} \geq 2$ & $\geq 4$ & - & $\geq 2 \mathrm{tH}, 5 \mathrm{j}, \geq 4 \mathrm{~b}$ \\
\hline
\end{tabular}

Table 2. Definition of the search and validation regions (see text for details) in the 1-lepton channel. 


\begin{tabular}{|c|c|c|c|c|c|}
\hline \multicolumn{6}{|c|}{ 0-lepton channel } \\
\hline \multicolumn{6}{|c|}{ Search regions $(\geq 7$ jets $)$} \\
\hline$N_{\mathrm{t}}$ & $N_{\mathrm{H}}$ & $b$-tag multiplicity & $m_{\mathrm{T}, \min }^{\mathrm{b}}$ & $m_{\text {eff }}$ & Channel name \\
\hline 0 & 0 & 2 & $>160 \mathrm{GeV}$ & $>1 \mathrm{TeV}$ & $0 \mathrm{t}, 0 \mathrm{H}, \geq 7 \mathrm{j}, 2 \mathrm{~b}, \mathrm{HM}$ \\
\hline 0 & 0 & 3 & $<160 \mathrm{GeV}$ & $>1 \mathrm{TeV}$ & $0 \mathrm{t}, 0 \mathrm{H}, \geq 7 \mathrm{j}, 3 \mathrm{~b}, \mathrm{LM}$ \\
\hline 0 & 0 & 3 & $>160 \mathrm{GeV}$ & $>1 \mathrm{TeV}$ & $0 \mathrm{t}, 0 \mathrm{H}, \geq 7 \mathrm{j}, 3 \mathrm{~b}, \mathrm{HM}$ \\
\hline 0 & 0 & $\geq 4$ & $<160 \mathrm{GeV}$ & $>1 \mathrm{TeV}$ & $0 \mathrm{t}, 0 \mathrm{H}, \geq 7 \mathrm{j}, \geq 4 \mathrm{~b}, \mathrm{LM}$ \\
\hline 0 & 0 & $\geq 4$ & $>160 \mathrm{GeV}$ & $>1 \mathrm{TeV}$ & $0 \mathrm{t}, 0 \mathrm{H}, \geq 7 \mathrm{j}, \geq 4 \mathrm{~b}, \mathrm{HM}$ \\
\hline 1 & 0 & $\overline{2}$ & $>160 \overline{\mathrm{GeV}}$ & $>1 \mathrm{TeV}$ & $1 \mathrm{t}, 0 \mathrm{H}, \geq 7 \mathrm{j}, 2 \mathrm{~b}, \mathrm{HM}$ \\
\hline 1 & 0 & 3 & $<160 \mathrm{GeV}$ & $>1 \mathrm{TeV}$ & $1 \mathrm{t}, 0 \mathrm{H}, \geq 7 \mathrm{j}, 3 \mathrm{~b}, \mathrm{LM}$ \\
\hline 1 & 0 & 3 & $>160 \mathrm{GeV}$ & $>1 \mathrm{TeV}$ & $1 \mathrm{t}, 0 \mathrm{H}, \geq 7 \mathrm{j}, 3 \mathrm{~b}, \mathrm{HM}$ \\
\hline 1 & 0 & $\geq 4$ & $<160 \mathrm{GeV}$ & $>1 \mathrm{TeV}$ & $1 \mathrm{t}, 0 \mathrm{H}, \geq 7 \mathrm{j}, \geq 4 \mathrm{~b}, \mathrm{LM}$ \\
\hline 1 & 0 & $\geq 4$ & $>160 \mathrm{GeV}$ & $>1 \mathrm{TeV}$ & $1 \mathrm{t}, 0 \mathrm{H}, \geq 7 \mathrm{j}, \geq 4 \mathrm{~b}, \mathrm{HM}$ \\
\hline 0 & $\overline{1}$ & $\overline{2}-$ & $>160 \overline{\mathrm{GeV}}$ & $>1 \mathrm{TeV}$ & $0 \mathrm{t}, 1 \mathrm{H}, \geq 7 \mathrm{j}, 2 \mathrm{~b}, \mathrm{HM}$ \\
\hline 0 & 1 & 3 & $<160 \mathrm{GeV}$ & $>1 \mathrm{TeV}$ & $0 \mathrm{t}, 1 \mathrm{H}, \geq 7 \mathrm{j}, 3 \mathrm{~b}, \mathrm{LM}$ \\
\hline 0 & 1 & 3 & $>160 \mathrm{GeV}$ & $>1 \mathrm{TeV}$ & $0 \mathrm{t}, 1 \mathrm{H}, \geq 7 \mathrm{j}, 3 \mathrm{~b}, \mathrm{HM}$ \\
\hline 0 & 1 & $\geq 4$ & $<160 \mathrm{GeV}$ & $>1 \mathrm{TeV}$ & $0 \mathrm{t}, 1 \mathrm{H}, \geq 7 \mathrm{j}, \geq 4 \mathrm{~b}, \mathrm{LM}$ \\
\hline 0 & 1 & $\geq 4$ & $>160 \mathrm{GeV}$ & $>1 \mathrm{TeV}$ & $0 \mathrm{t}, 1 \mathrm{H}, \geq 7 \mathrm{j}, \geq 4 \mathrm{~b}, \mathrm{HM}$ \\
\hline 1 & 1 & 3 & $<160 \mathrm{GeV}$ & $>1 \mathrm{TeV}$ & $1 \mathrm{t}, 1 \mathrm{H}, \geq 7 \mathrm{j}, 3 \mathrm{~b}, \mathrm{LM}$ \\
\hline 1 & 1 & 3 & $>160 \mathrm{GeV}$ & $>1 \mathrm{TeV}$ & $1 \mathrm{t}, 1 \mathrm{H}, \geq 7 \mathrm{j}, 3 \mathrm{~b}, \mathrm{HM}$ \\
\hline$\geq \overline{2}$ & 0 or 1 & $\overline{3}$ & $<160 \overline{\mathrm{GeV}}$ & $>1 \mathrm{TeV}$ & $\geq 2 \mathrm{t}, 0-1 \mathrm{H}, \geq 7 \mathrm{j}, 3 \mathrm{~b}, \mathrm{LM}$ \\
\hline$\geq 2$ & 0 or 1 & 3 & $>160 \mathrm{GeV}$ & $>1 \mathrm{TeV}$ & $\geq 2 \mathrm{t}, 0-1 \mathrm{H}, \geq 7 \mathrm{j}, 3 \mathrm{~b}, \mathrm{HM}$ \\
\hline$\geq 0$ & $\geq 2$ & 3 & & $>1 \mathrm{TeV}$ & $\geq 0 \mathrm{t}, \geq 2 \mathrm{H}, \geq 7 \mathrm{j}, 3 \mathrm{~b}$ \\
\hline$N_{\mathrm{t}}+$ & $N_{\mathrm{H}} \geq 2$ & 2 & $>160 \overline{\mathrm{GeV}}$ & $>1 \mathrm{TeV}$ & $\geq 2 \overline{\mathrm{t}}, \geq 7 \mathrm{j}, \overline{\mathrm{b}}, \overline{\mathrm{HM}}$ \\
\hline$N_{\mathrm{t}}+$ & $N_{\mathrm{H}} \geq 2$ & $\geq 4$ & - & $>1 \mathrm{TeV}$ & $\geq 2 \mathrm{tH}, \geq 7 \mathrm{j}, \geq 4 \mathrm{~b}$ \\
\hline \multicolumn{6}{|c|}{ Validation regions (6 jets) } \\
\hline$N_{\mathrm{t}}$ & $N_{\mathrm{H}}$ & $b$-tag multiplicity & $m_{\mathrm{T}, \min }^{\mathrm{b}}$ & $m_{\mathrm{eff}}$ & Channel name \\
\hline 0 & 0 & 2 & $>160 \mathrm{GeV}$ & $>1 \mathrm{TeV}$ & $0 \mathrm{t}, 0 \mathrm{H}, 6 \mathrm{j}, 2 \mathrm{~b}, \mathrm{HM}$ \\
\hline 0 & 0 & 3 & $<160 \mathrm{GeV}$ & $>1 \mathrm{TeV}$ & $0 \mathrm{t}, 0 \mathrm{H}, 6 \mathrm{j}, 3 \mathrm{~b}, \mathrm{LM}$ \\
\hline 0 & 0 & 3 & $>160 \mathrm{GeV}$ & $>1 \mathrm{TeV}$ & $0 \mathrm{t}, 0 \mathrm{H}, 6 \mathrm{j}, 3 \mathrm{~b}, \mathrm{HM}$ \\
\hline 0 & 0 & $\geq 4$ & $<160 \mathrm{GeV}$ & $>1 \mathrm{TeV}$ & $0 \mathrm{t}, 0 \mathrm{H}, 6 \mathrm{j}, \geq 4 \mathrm{~b}, \mathrm{LM}$ \\
\hline 0 & 0 & $\geq 4$ & $>160 \mathrm{GeV}$ & $>1 \mathrm{TeV}$ & $0 \mathrm{t}, 0 \mathrm{H}, 6 \mathrm{j}, \geq 4 \mathrm{~b}, \mathrm{HM}$ \\
\hline 1 & 0 & $\overline{2}-$ & $>160 \overline{\mathrm{GeV}}$ & $>1 \mathrm{TeV}$ & $1 \mathrm{t}, \overline{\mathrm{OH}}, \overline{6 \mathrm{j}}, \overline{2 \mathrm{~b}}, \overline{\mathrm{HM}}$ \\
\hline 1 & 0 & 3 & $<160 \mathrm{GeV}$ & $>1 \mathrm{TeV}$ & $1 \mathrm{t}, 0 \mathrm{H}, 6 \mathrm{j}, 3 \mathrm{~b}, \mathrm{LM}$ \\
\hline 1 & 0 & 3 & $>160 \mathrm{GeV}$ & $>1 \mathrm{TeV}$ & $1 \mathrm{t}, 0 \mathrm{H}, 6 \mathrm{j}, 3 \mathrm{~b}, \mathrm{HM}$ \\
\hline 1 & 0 & $\geq 4$ & 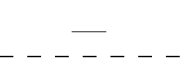 & $>1 \mathrm{TeV}$ & $1 \mathrm{t}, 0 \mathrm{H}, 6 \mathrm{j}, \geq 4 \mathrm{~b}$ \\
\hline 0 & 1 & $\overline{2}$ & $>160 \overline{\mathrm{GeV}}$ & $>1 \mathrm{TeV}$ & $0 \mathrm{t}, \overline{\mathrm{H}}, \overline{6 \mathrm{j}}, \overline{\mathrm{b}}, \overline{\mathrm{H}} \overline{\mathrm{M}}$ \\
\hline 0 & 1 & 3 & $<160 \mathrm{GeV}$ & $>1 \mathrm{TeV}$ & $0 \mathrm{t}, 1 \mathrm{H}, 6 \mathrm{j}, 3 \mathrm{~b}, \mathrm{LM}$ \\
\hline 0 & 1 & 3 & $>160 \mathrm{GeV}$ & $>1 \mathrm{TeV}$ & $0 \mathrm{t}, 1 \mathrm{H}, 6 \mathrm{j}, 3 \mathrm{~b}, \mathrm{HM}$ \\
\hline 0 & 1 & $\geq 4$ & $\ldots-\ldots$ & $>1 \mathrm{TeV}$ & $0 \mathrm{t}, 1 \mathrm{H}, 6 \mathrm{j}, \geq 4 \mathrm{~b}$ \\
\hline$\overline{N_{\mathrm{t}}}+$ & $N_{\mathrm{H}} \geq 2$ & $\overline{2}-$ & $>160 \overline{\mathrm{GeV}}$ & $>1 \mathrm{TeV}$ & $\geq 2 \mathrm{t} \overline{\mathrm{H}}, \overline{\mathrm{j}}, 2 \mathrm{~b}, \overline{\mathrm{H}} \overline{\mathrm{M}}$ \\
\hline$N_{\mathrm{t}}+$ & $N_{\mathrm{H}} \geq 2$ & 3 & - & $>1 \mathrm{TeV}$ & $\geq 2 \mathrm{tH}, 6 \mathrm{j}, 3 \mathrm{~b}$ \\
\hline$N_{\mathrm{t}}+$ & $N_{\mathrm{H}} \geq 2$ & $\geq 4$ & - & $>1 \mathrm{TeV}$ & $\geq 2 \mathrm{tH}, 6 \mathrm{j}, \geq 4 \mathrm{~b}$ \\
\hline
\end{tabular}

Table 3. Definition of the search and validation regions (see text for details) in the 0-lepton channel. 


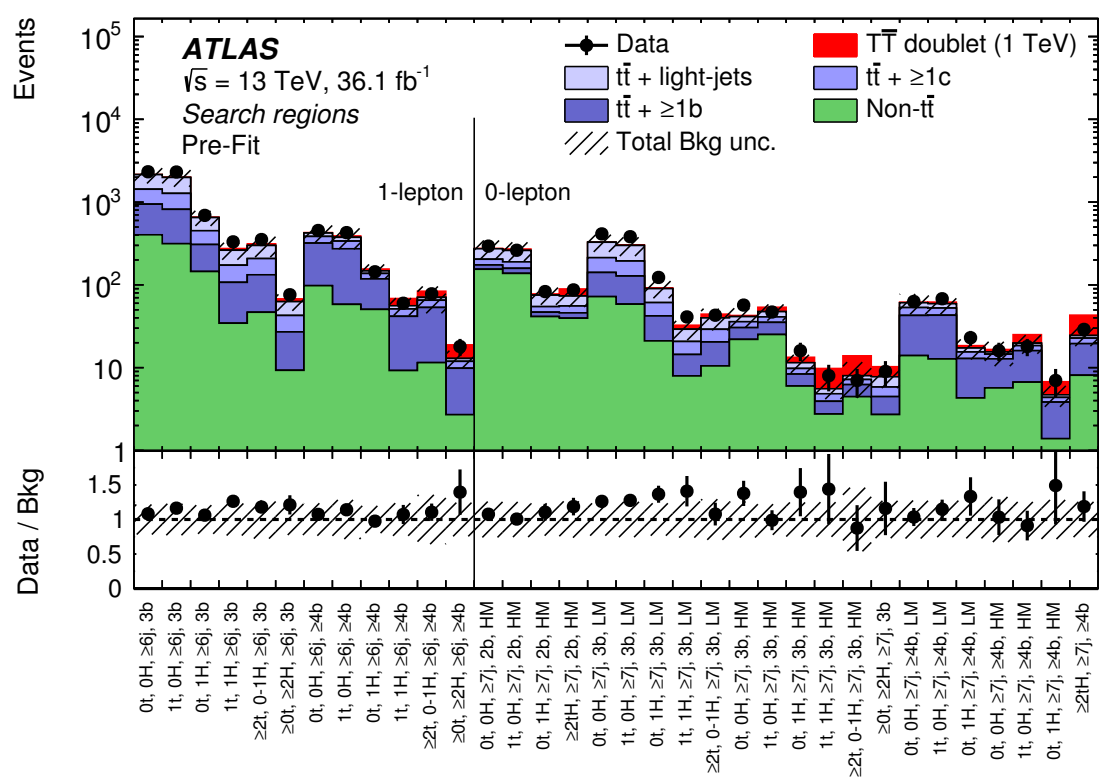

Figure 6. Comparison between the data and the background prediction for the yields in the search regions considered in the 1-lepton and 0-lepton channels, before the fit to data ("Pre-fit"). The small contributions from $t \bar{t} V, t \bar{t} H$, single-top, $W / Z+$ jets, diboson, and multijet backgrounds are combined into a single background source referred to as "Non- $t \bar{t}$ ". The expected $T \bar{T}$ signal (solid red) corresponding to $m_{T}=1 \mathrm{TeV}$ in the $T$ doublet scenario is also shown, added on top of the background prediction. The bottom panel displays the ratio of data to the SM background ("Bkg") prediction. The hashed area represents the total uncertainty of the background, excluding the normalisation uncertainty of the $t \bar{t}+\geq 1 b$ background, which is determined via a likelihood fit to data.

The leading sources of systematic uncertainty vary depending on the analysis region considered. For example, the total systematic uncertainty of the background normalisation in the highest-sensitivity search region in the 1-lepton channel $(\geq 0 \mathrm{t}, \geq 2 \mathrm{H}, \geq 6 \mathrm{j}, \geq 4 \mathrm{~b})$ is $25 \%$, with the largest contributions originating from uncertainties in $t \bar{t}+\mathrm{HF}$ modelling and flavour tagging efficiencies $(b, c$, and light). The above uncertainty does not include the uncertainty in the $t \bar{t}+\geq 1 b$ normalisation, which is allowed to vary freely in the fit to data. However, as discussed previously, the joint fit to data across the 34 search regions considered in total in the 1-lepton and 0-lepton channels allows the overall background uncertainty to be reduced significantly, e.g., in the case of the search region specified above, down to $10 \%$ (including the uncertainty in the $t \bar{t}+\geq 1 b$ normalisation). Such a reduction results from the significant constraints that the data places on some systematic uncertainties, as well as the correlations among systematic uncertainties built into the likelihood model.

The following sections describe the systematic uncertainties considered in this analysis.

\subsection{Luminosity}

The uncertainty in the integrated luminosity is $2.1 \%$, affecting the overall normalisation of all processes estimated from the simulation. It is derived, following a methodology 


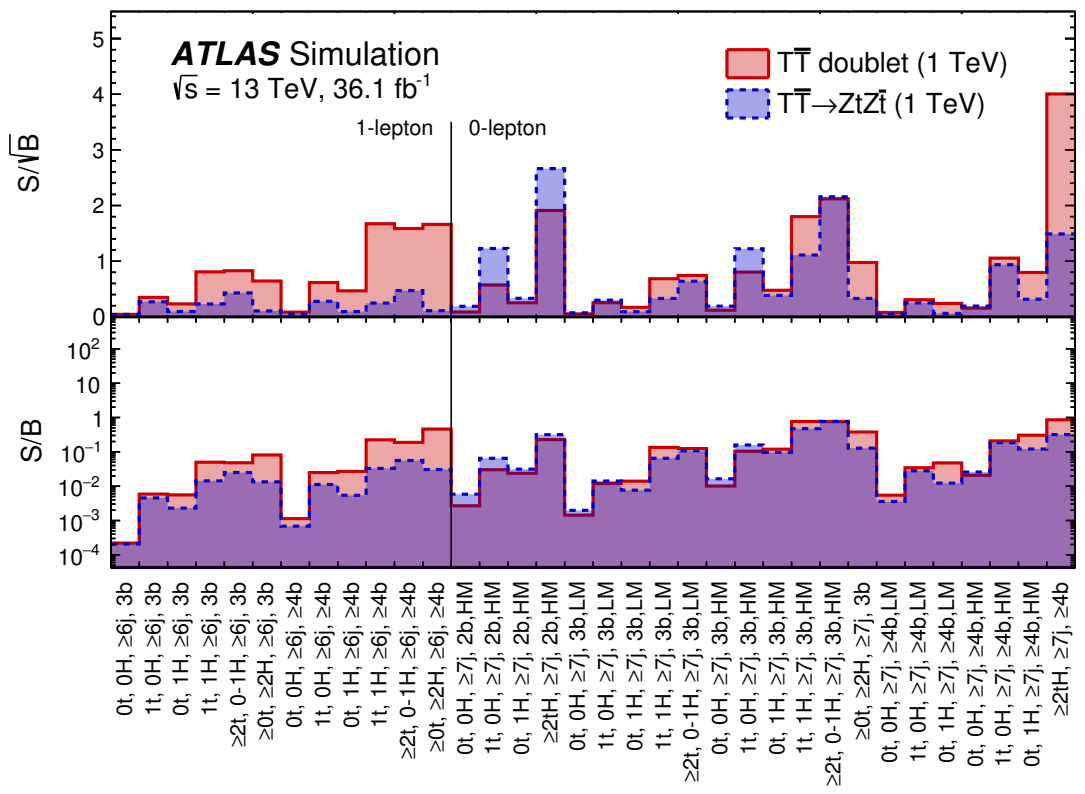

Figure 7. Signal-to-background ratio expressed as $S / \sqrt{B}$ (resp. $S / B$ ) in the top (resp. bottom) panel for each of the search regions. $B$ and $S$ stand for the total numbers of expected background and signal events in each region, respectively. For a $1 \mathrm{TeV} T$ quark mass hypothesis, two branching ratio configurations are displayed: the doublet model (red filled area) and $\mathcal{B}(T \rightarrow Z t)=1$ (blue filled area).

similar to that detailed in ref. [99], from a calibration of the luminosity scale using $x-y$ beam-separation scans performed in August 2015 and May 2016.

\subsection{Reconstructed objects}

Uncertainties associated with leptons arise from the trigger, reconstruction, identification, and isolation efficiencies, as well as the lepton momentum scale and resolution. These are measured in data using $Z \rightarrow \ell^{+} \ell^{-}$and $J / \psi \rightarrow \ell^{+} \ell^{-}$events [31,33]. The combined effect of all these uncertainties results in an overall normalisation uncertainty in signal and background of approximately $1 \%$.

Uncertainties associated with jets arise from the jet energy scale and resolution, and the efficiency to pass the JVT requirement. The largest contribution results from the jet energy scale, whose uncertainty dependence on jet $p_{\mathrm{T}}$ and $\eta$, jet flavour, and pile-up treatment is split into 21 uncorrelated components that are treated independently in the analysis [38].

The leading uncertainties associated with reconstructed objects in this analysis originate from the modelling of the $b_{-}, c_{-}$, and light-jet-tagging efficiencies in the simulation, which is corrected to match the efficiencies measured in data control samples [41]. Uncertainties in these corrections include a total of six independent sources affecting $b$-jets and four independent sources affecting $c$-jets. Each of these uncertainties has a different dependence on jet $p_{\mathrm{T}}$. Seventeen sources of uncertainty affecting light jets are considered, which depend on jet $p_{\mathrm{T}}$ and $\eta$. The sources of systematic uncertainty listed above are taken as uncorrelated between $b$-jets, $c$-jets, and light-jets. An additional uncertainty is included 


\begin{tabular}{|c|c|c|c|c|c|}
\hline 1-lepton channel & $\begin{array}{c}\geq 2 \mathrm{t}, 0-1 \mathrm{H}, \\
\geq 6 \mathrm{j}, 3 \mathrm{~b}\end{array}$ & $\begin{array}{c}1 \mathrm{t}, 0 \mathrm{H}, \\
\geq 6 \mathrm{j}, \geq 4 \mathrm{~b}\end{array}$ & $\begin{array}{c}1 \mathrm{t}, 1 \mathrm{H} \\
\geq 6 \mathrm{j}, \geq 4 \mathrm{~b}\end{array}$ & $\begin{array}{c}\geq 2 \mathrm{t}, 0-1 \mathrm{H} \\
\geq 6 \mathrm{j}, \geq 4 \mathrm{~b}\end{array}$ & $\begin{array}{l}\geq 0 \mathrm{t}, \geq 2 \mathrm{H}, \\
\geq 6 \mathrm{j}, \geq 4 \mathrm{~b}\end{array}$ \\
\hline \multicolumn{6}{|l|}{$\overline{T \bar{T}}\left(m_{T}=1 \mathrm{TeV}\right)$} \\
\hline $\mathcal{B}(T \rightarrow H t)=1$ & $19.6 \pm 1.5$ & $21.5 \pm 2.6$ & $24.3 \pm 2.7$ & $23.9 \pm 2.8$ & $14.6 \pm 2.0$ \\
\hline$T$ doublet & $14.2 \pm 1.0$ & $15.2 \pm 1.6$ & $12.5 \pm 1.4$ & $13.3 \pm 1.5$ & $5.96 \pm 0.62$ \\
\hline$T$ singlet & $7.88 \pm 0.58$ & $8.13 \pm 0.94$ & $5.47 \pm 0.62$ & $5.51 \pm 0.69$ & $2.18 \pm 0.23$ \\
\hline \multicolumn{6}{|l|}{$t \bar{t} t \bar{t}$} \\
\hline $\operatorname{EFT}\left(\left|C_{4 t}\right| / \Lambda^{2}=4 \pi \mathrm{TeV}^{-2}\right)$ & $535 \pm 30$ & $706 \pm 80$ & $171 \pm 19$ & $468 \pm 55$ & $34.3 \pm 5.0$ \\
\hline $2 \mathrm{UED} / \mathrm{RPP}\left(m_{\mathrm{KK}}=1.6 \mathrm{TeV}\right)$ & $9.77 \pm 0.46$ & $1.84 \pm 0.35$ & $1.00 \pm 0.19$ & $8.9 \pm 1.4$ & $0.39 \pm 0.09$ \\
\hline$t \bar{t}+$ light-jets & $91 \pm 46$ & $38 \pm 17$ & $4.8 \pm 2.4$ & $5.4 \pm 3.3$ & $0.99 \pm 0.49$ \\
\hline$t \bar{t}+\geq 1 c$ & $75 \pm 45$ & $64 \pm 38$ & $9.5 \pm 5.6$ & $11.8 \pm 7.5$ & $2.1 \pm 1.3$ \\
\hline$t \bar{t}+\geq 1 b$ & $86 \pm 41$ & $215 \pm 83$ & $32.4 \pm 9.5$ & $42 \pm 22$ & $7.1 \pm 2.2$ \\
\hline$t \bar{t} V$ & $9.7 \pm 1.8$ & $11.4 \pm 2.4$ & $1.73 \pm 0.39$ & $2.46 \pm 0.53$ & $0.41 \pm 0.10$ \\
\hline$t \bar{t} H$ & $4.90 \pm 0.78$ & $15.0 \pm 2.8$ & $3.79 \pm 0.65$ & $2.84 \pm 0.62$ & $1.19 \pm 0.20$ \\
\hline$W+$ jets & $9.4 \pm 4.4$ & $8.2 \pm 4.2$ & $0.69 \pm 0.50$ & $1.32 \pm 0.71$ & $0.54 \pm 0.48$ \\
\hline$Z+$ jets & $1.31 \pm 0.64$ & $0.95 \pm 0.48$ & $0.10 \pm 0.07$ & $0.13 \pm 0.08$ & $0.06 \pm 0.05$ \\
\hline Single top & $13.1 \pm 5.5$ & $16.6 \pm 7.0$ & $1.69 \pm 0.76$ & $1.97 \pm 0.95$ & $0.26 \pm 0.21$ \\
\hline Diboson & $1.8 \pm 1.1$ & $0.99 \pm 0.55$ & $0.11 \pm 0.09$ & $0.22 \pm 0.14$ & $0.01 \pm 0.04$ \\
\hline$t \bar{t} t \bar{t}(\mathrm{SM})$ & $2.82 \pm 0.86$ & $4.9 \pm 1.6$ & $1.12 \pm 0.36$ & $2.55 \pm 0.82$ & $0.23 \pm 0.07$ \\
\hline Total background & $299 \pm 83$ & $380 \pm 110$ & $56 \pm 13$ & $71 \pm 25$ & $12.9 \pm 3.2$ \\
\hline Data & 353 & 428 & 60 & 78 & 18 \\
\hline
\end{tabular}

Table 4. Predicted and observed yields in the 1-lepton channel in five of the most sensitive search regions (depending on the signal scenario) considered. The multijet background is estimated to be negligible in these regions and thus not shown. The background prediction is shown before the fit to data. Also shown are the signal predictions for different benchmark scenarios considered. The quoted uncertainties are the sum in quadrature of statistical and systematic uncertainties in the yields, excluding the normalisation uncertainty of the $t \bar{t}+\geq 1 b$ background, which is determined via a likelihood fit to data.

due to the extrapolation of these corrections to jets with $p_{\mathrm{T}}$ beyond the kinematic reach of the data calibration samples used $\left(p_{\mathrm{T}}>300 \mathrm{GeV}\right.$ for $b$ - and $c$-jets, and $p_{\mathrm{T}}>750 \mathrm{GeV}$ for light-jets); it is taken to be correlated among the three jet flavours. This uncertainty is evaluated in the simulation by comparing the tagging efficiencies while varying e.g. the fraction of tracks with shared hits in the silicon detectors or the fraction of fake tracks resulting from random combinations of hits, both of which typically increase at high $p_{\mathrm{T}}$ due to growing track multiplicity and density of hits within the jet. Finally, an uncertainty related to the application of $c$-jet scale factors to $\tau$-jets is considered, but has a negligible impact in this analysis. The combined effect of these uncertainties results in an uncertainty in the $t \bar{t}$ background normalisation ranging from $4 \%$ to $12 \%$ depending on the analysis region. The corresponding uncertainty range for signal is $2-12 \%$, assuming $T \bar{T}$ production in the weak-isospin doublet scenario and $m_{T}=1 \mathrm{TeV}$. 


\begin{tabular}{|lccccc|}
\hline 0-lepton channel & $\begin{array}{c}\geq \mathrm{tH}, \\
\geq 7 \mathrm{j}, 2 \mathrm{~b}, \mathrm{HM}\end{array}$ & $\begin{array}{c}1 \mathrm{t}, 1 \mathrm{H}, \\
\geq 7 \mathrm{j}, 3 \mathrm{~b}, \mathrm{HM}\end{array}$ & $\begin{array}{c}\geq 2 \mathrm{t}, 0-1 \mathrm{H}, \\
\geq 7 \mathrm{j}, 3 \mathrm{~b}, \mathrm{HM}\end{array}$ & $\begin{array}{c}1 \mathrm{t}, 0 \mathrm{H}, \\
\geq 4 \mathrm{j}, \mathrm{b}, \mathrm{HM}\end{array}$ & $\begin{array}{c}\geq 2 \mathrm{tH}, \\
\geq 7 \mathrm{j}, \geq 4 \mathrm{~b}\end{array}$ \\
\hline$T \bar{T}\left(m_{T}=1 \mathrm{TeV}\right)$ & & & & & \\
$\mathcal{B}(T \rightarrow Z t)=1$ & $22.3 \pm 2.3$ & $2.60 \pm 0.57$ & $6.02 \pm 0.61$ & $4.72 \pm 0.66$ & $6.94 \pm 0.98$ \\
$T$ doublet & $16.0 \pm 1.1$ & $4.22 \pm 0.34$ & $5.92 \pm 0.49$ & $5.32 \pm 0.61$ & $18.7 \pm 2.0$ \\
$T$ singlet & $8.52 \pm 0.61$ & $1.81 \pm 0.16$ & $2.63 \pm 0.22$ & $2.32 \pm 0.29$ & $6.91 \pm 0.80$ \\
\hline$t \bar{t}+$ light-jets & $17.8 \pm 9.8$ & $0.72 \pm 0.40$ & $0.80 \pm 0.53$ & $1.30 \pm 0.72$ & $1.71 \pm 0.98$ \\
$t \bar{t}+\geq 1 c$ & $9.7 \pm 6.4$ & $0.92 \pm 0.65$ & $0.95 \pm 0.71$ & $2.4 \pm 1.6$ & $3.2 \pm 2.0$ \\
$t \bar{t}+\geq 1 b$ & $6.3 \pm 4.2$ & $1.17 \pm 0.59$ & $1.78 \pm 0.74$ & $9.4 \pm 3.2$ & $11.4 \pm 4.1$ \\
$t \bar{t} V$ & $5.5 \pm 1.0$ & $0.49 \pm 0.12$ & $0.88 \pm 0.19$ & $1.19 \pm 0.27$ & $1.01 \pm 0.24$ \\
$t \bar{t} H$ & $0.61 \pm 0.12$ & $0.17 \pm 0.05$ & $0.13 \pm 0.04$ & $0.85 \pm 0.17$ & $1.08 \pm 0.25$ \\
$W+$ jets & $9.6 \pm 4.1$ & $0.52 \pm 0.27$ & $0.80 \pm 0.37$ & $0.81 \pm 0.40$ & $0.56 \pm 0.28$ \\
$Z+$ jets & $8.6 \pm 4.5$ & $0.59 \pm 0.28$ & $0.8 \pm 2.1$ & $0.80 \pm 0.40$ & $0.63 \pm 0.42$ \\
Single top & $8.3 \pm 4.4$ & $0.69 \pm 0.43$ & $0.97 \pm 0.59$ & $1.8 \pm 1.0$ & $1.10 \pm 0.61$ \\
Diboson & $2.9 \pm 1.9$ & $0.11 \pm 0.20$ & $0.55 \pm 0.66$ & $0.24 \pm 0.25$ & $0.14 \pm 0.15$ \\
$t \bar{t} t \bar{t}$ (SM) & $0.22 \pm 0.07$ & $0.06 \pm 0.02$ & $0.12 \pm 0.04$ & $0.31 \pm 0.10$ & $0.77 \pm 0.25$ \\
Multijet & $3.9 \pm 3.9$ & $0.13 \pm 0.17$ & $0.20 \pm 0.24$ & $0.64 \pm 0.68$ & $2.8 \pm 2.8$ \\
\hline Total background & $73 \pm 19$ & $5.6 \pm 1.4$ & $8.0 \pm 3.7$ & $19.7 \pm 5.0$ & $24.4 \pm 6.3$ \\
\hline Data & 87 & 8 & 7 & 18 & 29 \\
\hline
\end{tabular}

Table 5. Predicted and observed yields in the 0-lepton channel in five of the most sensitive search regions (depending on the signal scenario) considered. The background prediction is shown before the fit to data. Also shown are the signal predictions for different benchmark scenarios considered. The quoted uncertainties are the sum in quadrature of statistical and systematic uncertainties in the yields, excluding the normalisation uncertainty of the $t \bar{t}+\geq 1 b$ background, which is determined via a likelihood fit to data.

\subsection{Background modelling}

A number of sources of systematic uncertainty affecting the modelling of $t \bar{t}+$ jets are considered. An uncertainty of $6 \%$ is assigned to the inclusive $t \bar{t}$ production cross section [56], including contributions from varying the factorisation and renormalisation scales, and from uncertainties in the PDF, $\alpha_{\mathrm{S}}$, and the top quark mass, all added in quadrature. Since several search regions have a sufficiently large number of events of $t \bar{t}+\geq 1 b$ background, its normalisation is completely determined by the data during the fit procedure. In the case of the $t \bar{t}+\geq 1 c$ normalisation, since the fit to the data is unable to precisely determine it and the analysis has very limited sensitivity to its uncertainty, a normalisation uncertainty of $50 \%$ is assumed.

Alternative $t \bar{t}$ samples were generated using PowHEG-Box interfaced to HeRwIG++ 2.7.1 [100] and MG5_aMC 2.2.1 interfaced to HerwiG++ 2.7.1 in order to estimate systematic uncertainties related to the modelling of this background. The effects of initial- and final-state radiation (ISR/FSR) are explored using two alternative PowHEGBoX + PythiA samples, one with $h_{\text {damp }}$ set to $2 m_{t}$, the renormalisation and factorisation 
scales set to half the nominal value and using the P2012 radHi UE tune, giving more radiation (referred to as "radHi"), and one with the P2012 radLo UE tune, $h_{\text {damp }}=m_{t}$ and the renormalisation and factorisation scales set to twice the nominal value, giving less radiation (referred to as "radLow") [101].

Uncertainties affecting the modelling of $t \bar{t}+\geq 1 b$ production include shape uncertainties (including inter-category migration effects) associated with the NLO prediction from SherpaOL, which is used for reweighting the nominal Powheg-Box+Pythia $6 t \bar{t}+\geq 1 b$ prediction. These uncertainties include different scale variations, a different shower-recoil model scheme, and two alternative PDF sets (see ref. [102] for details), and are significantly smaller than those estimated by comparing different event generators. An uncertainty due to the choice of generator is assessed by comparing the $t \bar{t}+\geq 1 b$ predictions obtained after reweighting Powheg-Box+Pythia 6 to the NLO calculation from SherpaOL and to an equivalent NLO calculation from MG5_aMC+PyTHIA 8, which differs in the procedure used to match the NLO matrix element calculation and the parton shower (see section 1.6.8 of ref. [103]). The uncertainty from the parton shower and hadronisation model is taken from the difference between the MG5_aMC calculation showered with either PYTHIA8 or HeRwIG++. Additional uncertainties are assessed for the contributions to the $t \bar{t}+\geq 1 b$ background originating from multiple parton interactions or final-state radiation from top quark decay products, which are not part of the NLO prediction. The latter are assessed via the alternative "radHi" and "radLow" samples, as discussed below. The nominal NLO corrections, as well as their variations used to propagate the theoretical uncertainties in the NLO prediction, are adjusted so that the particle-level cross section of the $t \bar{t}+\geq 1 b$ background (i.e. prior to reconstruction-level selection requirements) is fixed to the nominal prediction, i.e. effectively only migrations across categories and distortions to the shape of the kinematic distributions are considered.

In the following, uncertainties affecting all $t \bar{t}+$ jets processes are discussed. Uncertainties associated with the modelling of ISR/FSR are obtained from the comparison of the Powheg-Box+Pythia 6 "radHi" and "radLow" samples (see section 5.2) with the nominal Powheg-Box+Pythia 6 sample. An uncertainty associated with the choice of NLO generator is derived by comparing two $t \bar{t}$ samples, one generated with PowHEGBox+HeRwIG++ and another generated with MG5_aMC+HERWIG++, and propagating the resulting fractional difference to the nominal PowhEG-BoX+PyThIA 6 prediction. An uncertainty due to the choice of parton shower and hadronisation model is derived by comparing events produced by Powheg-Box interfaced to Pythia 6 or HeRwig++. Finally, the uncertainty in the modelling of the top quark's $p_{\mathrm{T}}$, affecting only the $t \bar{t}+$ lightjets and $t \bar{t}+\geq 1 c$ processes, is evaluated by taking the full difference between applying and not applying the reweighting to match the NNLO prediction. The above uncertainties are taken as uncorrelated between the $t \bar{t}+$ light-jets, $t \bar{t}+\geq 1 c$ and $t \bar{t}+\geq 1 b$ processes. In the case of $t \bar{t}+\geq 1 b$, in all instances the various HF categories and the corresponding partonic kinematics for the alternative MC samples are reweighted to match the NLO prediction of SHERPAOL so that only effects other than distortions to the inter-normalisation of the various $t \bar{t}+\geq 1 b$ topologies and their parton-level kinematics are propagated. In the case of $t \bar{t}+$ light-jets and $t \bar{t}+\geq 1 c$ the full effect of these uncertainties is propagated. Similarly 
to the treatment of the NLO corrections and uncertainties associated with $t \bar{t}+\geq 1 b$ discussed above, in the case of the additional uncertainties derived by comparing alternative $t \bar{t}$ samples, the overall normalisation of the $t \bar{t}+\geq 1 c$ and $t \bar{t}+\geq 1 b$ background at the particle level is fixed to the nominal prediction. In this way, only migrations across categories and distortions to the shape of the kinematic distributions are considered. In order to maintain the inclusive $t \bar{t}$ cross section, the $t \bar{t}+$ light-jets background is adjusted accordingly.

Uncertainties affecting the modelling of the single-top-quark background include a $+5 \% /-4 \%$ uncertainty in the total cross section estimated as a weighted average of the theoretical uncertainties in $t$-, $W t$ - and $s$-channel production [84-86]. Additional uncertainties associated with the modelling of ISR/FSR are assessed by comparing the nominal samples with alternative samples where generator parameters were varied (i.e. "radHi" and "radLow"). For the $t$ - and $W t$-channel processes, an uncertainty due to the choice of parton shower and hadronisation model is derived by comparing events produced by POWHEG-Box interfaced to Pythia 6 or Herwig++. These uncertainties are treated as fully correlated among single-top production processes, but uncorrelated with the corresponding uncertainty in the $t \bar{t}+$ jets background. The sum in quadrature of the above uncertainties on the single top normalisation at the preselection level is $20 \%$ in the 1-lepton channel and $20 \%(25 \%)$ in $\mathrm{LM}(\mathrm{HM})$ regions of the 0-lepton channel, respectively. An additional systematic uncertainty on $W t$-channel production concerning the separation between $t \bar{t}$ and $W t$ at NLO [104] is assessed by comparing the nominal sample, which uses the so-called "diagram subtraction" scheme, with an alternative sample using the "diagram removal" scheme. This uncertainty, which is taken to be single-sided, has a strong shape dependence and affects the $W t$ normalisation by about $-50 \%$ in the 1-lepton channel and LM regions of the 0-lepton channel, and by about $-75 \%$ in HM regions of the 0-lepton channel. Due to the small size of the simulated samples, and hence limited statistical precision, these uncertainties cannot be reliably estimated in each analysis region and so their estimates at the preselection level are used instead. They are treated as uncorrelated across regions with different top-tagged jet and Higgs-tagged jet multiplicities and between the 1-lepton and 0-lepton channels.

Uncertainties affecting the normalisation of the $V+$ jets background are estimated for the sum of $W+$ jets and $Z+$ jets, and separately for $V+$ light-jets, $V+\geq 1 c+$ jets, and $V+\geq 1 b+$ jets subprocesses. The total normalisation uncertainty of $V+$ jets processes is estimated by comparing the data and total background prediction in the different analysis regions considered, but requiring exactly $0 b$-tagged jets. Agreement between data and predicted background in these modified regions, which are dominated by $V+$ light-jets, is found to be within approximately $30 \%$. This bound is taken to be the normalisation uncertainty, correlated across all $V+$ jets subprocesses. Since SHERPA 2.2 has been found to underestimate $V+$ heavy-flavour by about a factor of 1.3 [105], additional $30 \%$ normalisation uncertainties are assumed for $V+\geq 1 c+$ jets and $V+\geq 1 b+$ jets subprocesses, considered uncorrelated between them. These uncertainties are treated as uncorrelated across regions with different top-/Higgs-tagged jet multiplicities and between the 1-lepton and 0-lepton channels.

Uncertainties in the diboson background normalisation include $5 \%$ from the NLO theory cross sections [106], as well as an additional $24 \%$ normalisation uncertainty added in quadrature for each additional inclusive jet-multiplicity bin, based on a comparison among 
different algorithms for merging LO matrix elements and parton showers [107]. Therefore, normalisation uncertainties of $5 \% \oplus \sqrt{3} \times 24 \%=42 \%$ and $5 \% \oplus \sqrt{4} \times 24 \%=48 \%$ are assigned for events with exactly 5 jets and $\geq 6$ jets, respectively (this assumes that two jets come from the $W / Z$ decay, as in $W W / W Z \rightarrow \ell \nu j j$ ). Recent comparisons between data and SHERPA 2.1.1 for $W Z(\rightarrow \ell \nu \ell \ell)+\geq 4$ jets show agreement within the experimental uncertainty of approximately $40 \%$ [108], which further justifies the above uncertainty. This uncertainty is taken to be uncorrelated across regions with different top-/Higgs-tagged jet multiplicities and between the 1-lepton and 0-lepton channels

Uncertainties in the $t \bar{t} V$ and $t \bar{t} H$ cross sections are $15 \%$ and $+10 \% /-13 \%$, respectively, from the uncertainties in their respective NLO theoretical cross sections [109-111]. Finally, an uncertainty of $30 \%$ is estimated for the NLO prediction of the SM $t \bar{t} t \bar{t}$ cross section [67]. Since no additional modelling uncertainties are taken into account for these backgrounds, and the 1-lepton and 0-lepton channels cover different kinematic phase spaces, the above uncertainties in the $t \bar{t} V, t \bar{t} H$, and SM $t \bar{t} t \bar{t}$ cross sections are taken to be uncorrelated between the two channels.

Uncertainties in the data-driven multijet background estimate receive contributions from the limited sample size in data, particularly at high jet and $b$-tag multiplicities, as well as from the uncertainty in the misidentified-lepton rate, measured in different control regions (e.g. selected with a requirement on either the maximum $E_{\mathrm{T}}^{\text {miss }}$ or $m_{\mathrm{T}}^{W}$ ). Based on the comparisons between data and total prediction in multijet-rich selections, the normalisation uncertainties assumed for this background are $50 \%(100 \%)$ for electrons with $\left|\eta_{\text {cluster }}\right| \leq 1\left(\left|\eta_{\text {cluster }}\right|>1\right)$, and $50 \%$ for muons, taken to be uncorrelated across regions with different top-/Higgs-tagged jet multiplicities and between events containing electrons and events containing muons. In the case of the 0 -lepton channel, the normalisation uncertainty assigned to the multijet background is $100 \%$. No explicit shape uncertainty is assigned since the large statistical uncertainties associated with the multijet background prediction, which are uncorrelated between bins in the final discriminant distribution, are assumed to effectively cover possible shape uncertainties.

\section{Statistical analysis}

For each search, the $m_{\text {eff }}$ distributions across all regions considered are jointly analysed to test for the presence of a signal predicted by the benchmark scenarios. The statistical analysis uses a binned likelihood function $\mathcal{L}(\mu, \theta)$ constructed as a product of Poisson probability terms over all bins considered in the search. This function depends on the signal-strength parameter $\mu$, which multiplies the predicted production cross section for signal, and $\theta$, a set of nuisance parameters that encode the effect of systematic uncertainties in the signal and background expectations. Therefore, the expected total number of events in a given bin depends on $\mu$ and $\theta$. With the exception of the parameter that controls the normalisation of the $t \bar{t}+\geq 1 b$ background, all other nuisance parameters are implemented in the likelihood function as Gaussian or log-normal constraints. The above-mentioned $t \bar{t}+\geq 1 b$ normalisation factor is a free parameter of the fit. 
For a given value of $\mu$, the nuisance parameters $\theta$ allow variations of the expectations for signal and background according to the corresponding systematic uncertainties, and their fitted values result in the deviations from the nominal expectations that globally provide the best fit to the data. This procedure allows a reduction of the impact of systematic uncertainties on the search sensitivity by taking advantage of the highly populated background-dominated regions included in the likelihood fit. To verify the improved background prediction, fits under the background-only hypothesis are performed, and differences between the data and the post-fit background prediction are checked using kinematic variables other than the ones used in the fit. The $m_{\text {eff }}$ distributions in validation regions not used in the fit are also checked. Statistical uncertainties in each bin of the predicted $m_{\text {eff }}$ distributions due to the limited size of the simulated samples are taken into account by dedicated parameters in the fit.

The test statistic $q_{\mu}$ is defined as the profile likelihood ratio: $q_{\mu}=$ $-2 \ln \left(\mathcal{L}\left(\mu, \hat{\hat{\theta}}_{\mu}\right) / \mathcal{L}(\hat{\mu}, \hat{\theta})\right)$, where $\hat{\mu}$ and $\hat{\theta}$ are the values of the parameters that maximise the likelihood function (subject to the constraint $0 \leq \hat{\mu} \leq \mu$ ), and $\hat{\hat{\theta}}_{\mu}$ are the values of the nuisance parameters that maximise the likelihood function for a given value of $\mu$. The test statistic $q_{\mu}$ is evaluated with the RooFit package $[112,113]$. A related statistic is used to determine the probability that the observed data are compatible with the backgroundonly hypothesis (i.e. the discovery test) by setting $\mu=0$ in the profile likelihood ratio and leaving $\hat{\mu}$ unconstrained: $q_{0}=-2 \ln \left(\mathcal{L}\left(0, \hat{\hat{\theta}}_{0}\right) / \mathcal{L}(\hat{\mu}, \hat{\theta})\right)$. The $p$-value (referred to as $p_{0}$ ) representing the probability of the data being compatible with the background-only hypothesis is estimated by integrating the distribution of $q_{0}$ from background-only pseudoexperiments, approximated using the asymptotic formulae given in refs. [114], above the observed value of $q_{0}$. Some model dependence exists in the estimation of the $p_{0}$, as a given signal scenario needs to be assumed in the calculation of the denominator of $q_{\mu}$, even if the overall signal normalisation is left floating and fitted to data. The observed $p_{0}$ is checked for each explored signal scenario. Upper limits on the signal production cross section for each of the signal scenarios considered are derived by using $q_{\mu}$ in the $\mathrm{CL}_{\mathrm{s}}$ method $[115,116]$. For a given signal scenario, values of the production cross section (parameterised by $\mu$ ) yielding $\mathrm{CL}_{\mathrm{s}}<0.05$, where $\mathrm{CL}_{\mathrm{s}}$ is computed using the asymptotic approximation [114], are excluded at $\geq 95 \%$ CL.

\section{Results}

This section presents the results obtained from searches in the 1-lepton and 0-lepton channels, as well as their combination, following the statistical analysis discussed in section 8 .

\subsection{Likelihood fits to data}

A binned likelihood fit under the background-only hypothesis is performed on the $m_{\text {eff }}$ distributions in all search regions considered. In this section, the results of the simultaneous likelihood fit to the search regions in the 1-lepton and 0-lepton channels are discussed. This combined fit is used to obtain results on $T \bar{T}$ production. In this combination, all common 


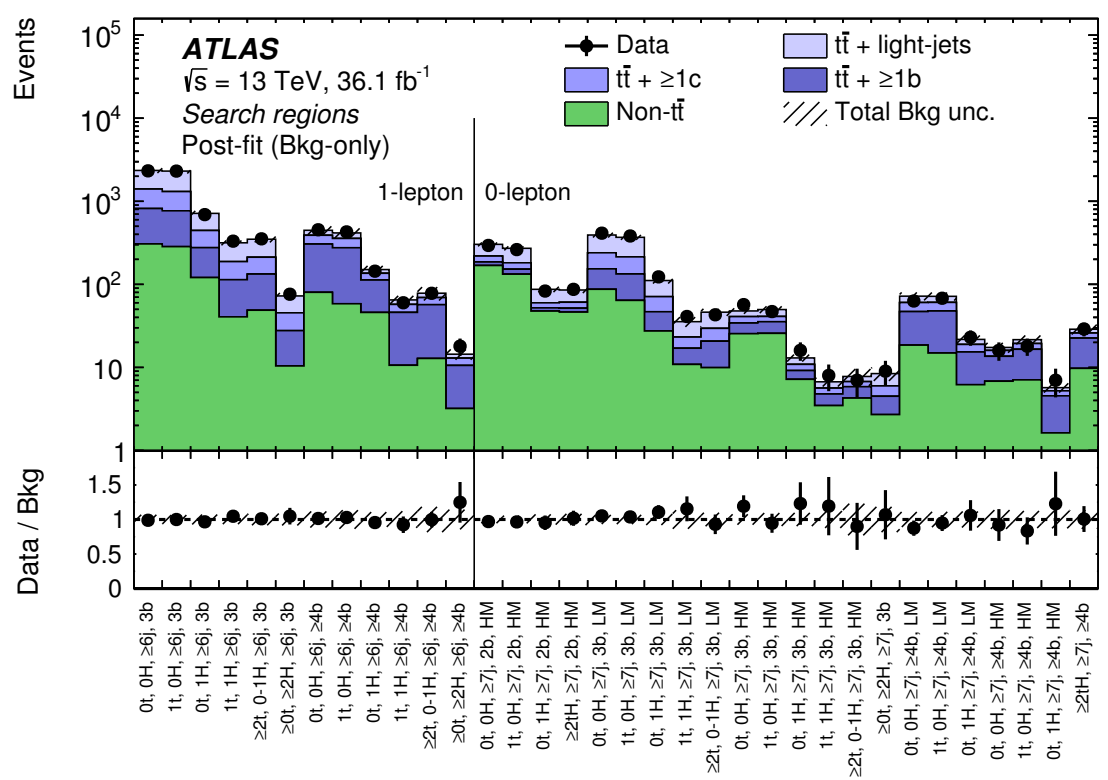

Figure 8. Comparison between the data and the background prediction for the yields in the search regions considered in the 1-lepton and 0-lepton channels, after the combined fit to data ("Post-fit") under the background-only hypothesis. The small contributions from $t \bar{t} V, t \bar{t} H$, singletop, $W / Z+$ jets, diboson, and multijet backgrounds are combined into a single background source referred to as "Non- $t \bar{t}$ ". The bottom panel displays the ratio of data to the SM background ("Bkg") prediction. The hashed area represents the total uncertainty of the background.

systematic uncertainties are considered fully correlated between the 1-lepton and 0-lepton channels, with the exception of those affecting non-t $t \bar{t}$ backgrounds. To obtain the results in the individual channels, separate fits are performed. In general, good agreement is found among the fitted nuisance parameters in the individual and combined fits.

A comparison of the distribution of observed and expected yields in the search regions in the 1-lepton and 0-lepton channels after the combined fit is shown in figure 8 (see figure 6 for the results before the combined fit). The post-fit yields in five of the most sensitive search regions in the 1-lepton and 0-lepton channels can be found in tables 6 and 7, respectively. For the same search regions, the corresponding $m_{\text {eff }}$ distributions, both before and after the fit to data, are shown in figures $9-13$. The binning used for the $m_{\text {eff }}$ distributions in the different search regions represents a compromise between preserving enough discrimination between the background and the different signal hypotheses considered, and keeping the statistical uncertainty on the background prediction per bin well below $30 \%$. While some of the systematic uncertainties from individual sources described in section 7 vary across the $m_{\text {eff }}$ spectrum, the total pre-fit uncertainty is largely independent of $m_{\mathrm{eff}}$. The large number of events in the signal-depleted regions, together with their different background compositions, and the assumptions of the fit model, constrain the combined effect of the sources of systematic uncertainty. As a result, an improved background prediction is obtained with significantly reduced uncertainty, not only in the signal-depleted channels, but also in the signal-rich channels such as $(\geq 0 \mathrm{t}, \geq 2 \mathrm{H}, \geq 6 \mathrm{j}, \geq 4 \mathrm{~b})$ in the 1-lepton channel. 
In the combined fit, the channels with three $b$-tagged jets are effectively used to constrain the leading uncertainties affecting the $t \bar{t}+$ light-jets background prediction, while the channels with $\geq 4 b$-tagged jets are sensitive to the uncertainties affecting the $t \bar{t}+\mathrm{HF}$ background prediction. In particular, one of the main corrections determined in the fit is a scale factor that multiplies the $t \bar{t}+\geq 1 b$ normalisation by $0.90 \pm 0.23$ relative to the nominal prediction. ${ }^{6}$ In addition, the nuisance parameter controlling the $t \bar{t}+\geq 1 c$ normalisation is adjusted to scale this background by a factor of $1.3 \pm 0.4$ relative to its nominal prediction. The fit results in better agreement between data and prediction in the channels with $\geq 3$-tagged jets, where the $t \bar{t}+\mathrm{HF}$ background dominates. Detailed studies were performed to verify the stability of the fit against variations in the treatment of the systematic uncertainties affecting the $t \bar{t}+\mathrm{HF}$ background (e.g. by decorrelating normalisation and shape uncertainties between different $t \bar{t}+\geq 1 b$ categories, or by scaling the $t \bar{t}+\geq 1 b$ and $t \bar{t}+\geq 1 c$ backgrounds by a common factor), finding in all instances a robust post-fit background prediction. Furthermore, the impact on the background-only fit of injecting a $T \bar{T}$ signal (with $m_{T}=1 \mathrm{TeV}$ ) in the doublet configuration was confirmed to be negligible. Although there is no single nuisance parameter directly responsible for the normalisation of $t \bar{t}+$ light-jets background, the yields for this contribution within each region are affected by systematic uncertainties in the $t \bar{t}$ modelling and the jet flavour tagging, and thus are changed after the fit.

A comparison of the distribution of observed and expected yields in all validation regions considered, before and after the combined fit in the search regions, is shown in figure 14. Agreement between data and prediction in normalisation and shape of the $m_{\mathrm{eff}}$ distribution for these regions, which are not used in the fit, is generally improved after the fit, giving confidence in the overall procedure. To increase the background yields and strengthen the validation of the fit strategy, comparisons between data and background prediction, before and after the fit, are performed for more-inclusive event selections. As an example, the distributions of two kinematic variables used to define the search strategy can be found in figures 15 and 16. They display respectively the Higgs-tagged jet multiplicity in the 1-lepton channel, after requiring at least 6 jets and $3 b$-jets, and the distribution of the $m_{\mathrm{T}}^{\mathrm{b}}$ min variable in the 0-lepton channel for events containing at least 7 jets and $2 b$-jets, together with at least one top/Higgs-tagged jet. Although these variables are not directly used in the fit, a good description of the data by the post-fit background prediction is observed, which further validates the fitting procedure. The result of the background-only fit to data is used for the background prediction in the computation of the limits presented in the following subsections.

\subsection{Limits on vector-like quark pair production}

No significant excess above the SM expectation is found in any of the search regions. Upper limits at $95 \%$ CL on the $T \bar{T}$ production cross section are set in several benchmark scenarios as a function of the $T$ quark mass $m_{T}$ and are compared to the theoretical prediction from Top++. The resulting lower limits on $m_{T}$ correspond to the central value of the

\footnotetext{
${ }^{6}$ Even though the $t \bar{t}+\geq 1 b$ normalisation factor is assumed to be the same in all regions, the overall change in $t \bar{t}+\geq 1 b$ normalisation can be different across channels due to the different impact of other nuisance parameters affecting the $t \bar{t}+\geq 1 b$ background, such as those related to $t \bar{t}+\geq 1 b$ modelling.
} 


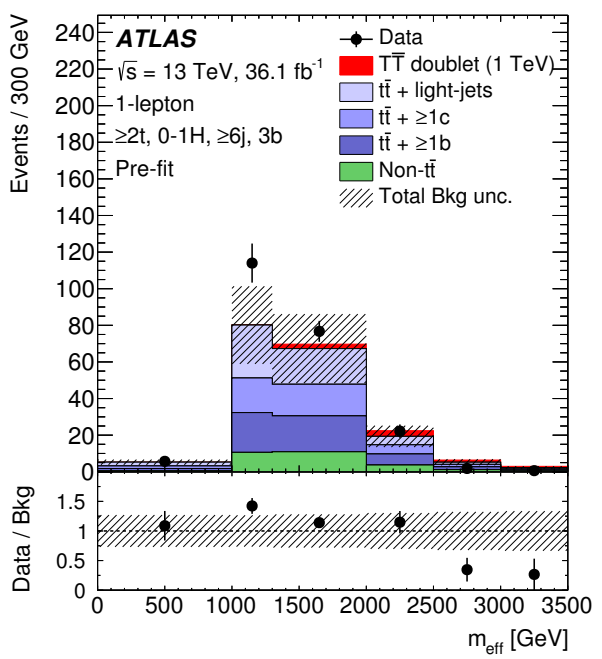

(a)

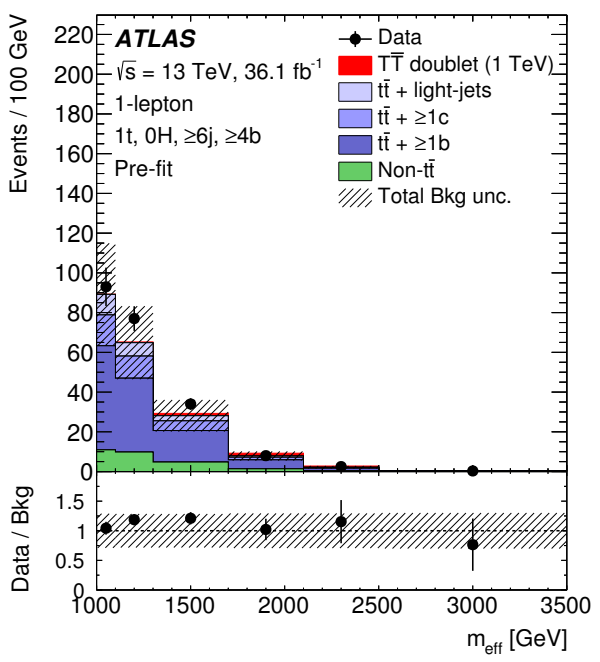

(c)

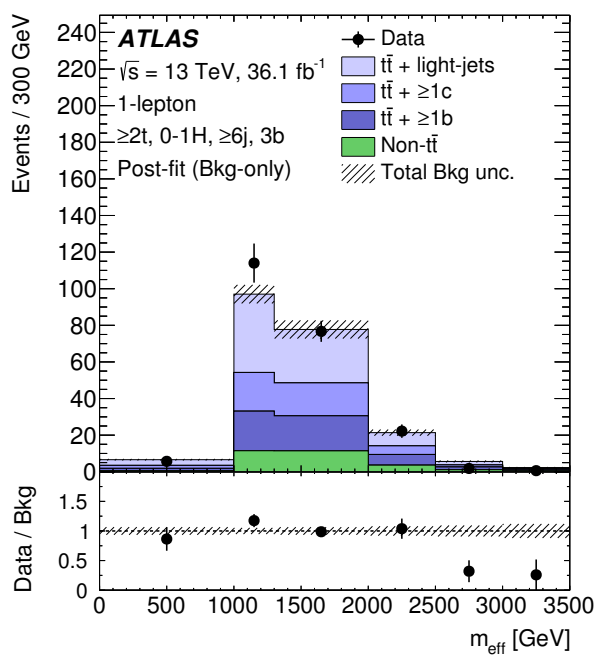

(b)

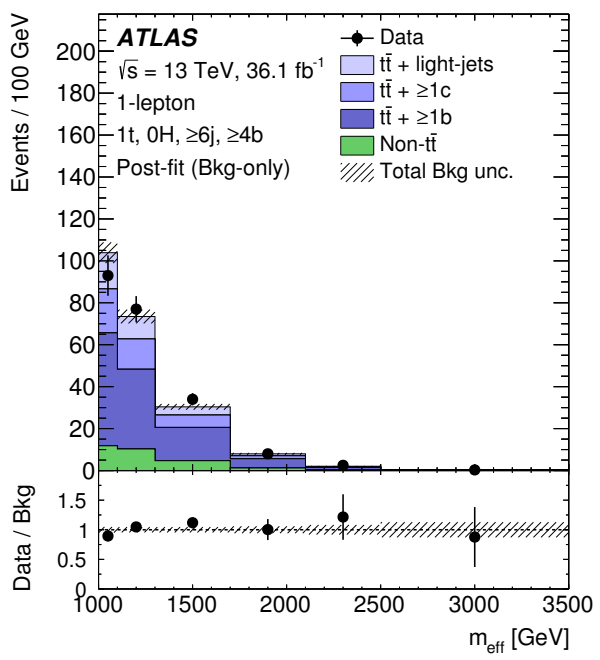

(d)

Figure 9. Comparison between the data and prediction for the $m_{\text {eff }}$ distribution in some of the most sensitive search regions in the 1-lepton channel, before and after performing the combined fit to data in the 0-lepton and 1-lepton channels ("Pre-fit" and "Post-fit", respectively) under the background-only hypothesis. Shown are the $(\geq 2 \mathrm{t}, 0-1 \mathrm{H}, \geq 6 \mathrm{j}, 3 \mathrm{~b})$ region (a) pre-fit and (b) post-fit, and the $(1 \mathrm{t}, 0 \mathrm{H}, \geq 6 \mathrm{j}, \geq 4 \mathrm{~b})$ region (c) pre-fit and (d) post-fit. In the pre-fit figures the expected $T \bar{T}$ signal (solid red) corresponding to $m_{T}=1 \mathrm{TeV}$ in the $T$ doublet scenario is also shown, added on top of the background prediction. The small contributions from $t \bar{t} V, t \bar{t} H$, single-top, $W / Z+$ jets, diboson, and multijet backgrounds are combined into a single background source referred to as "Non- $t \bar{t}$ ". The last bin in all figures contains the overflow. The bottom panels display the ratios of data to the total background prediction ("Bkg"). The hashed area represents the total uncertainty of the background. In the case of the pre-fit background uncertainty, the normalisation uncertainty of the $t \bar{t}+\geq 1 b$ background is not included. 


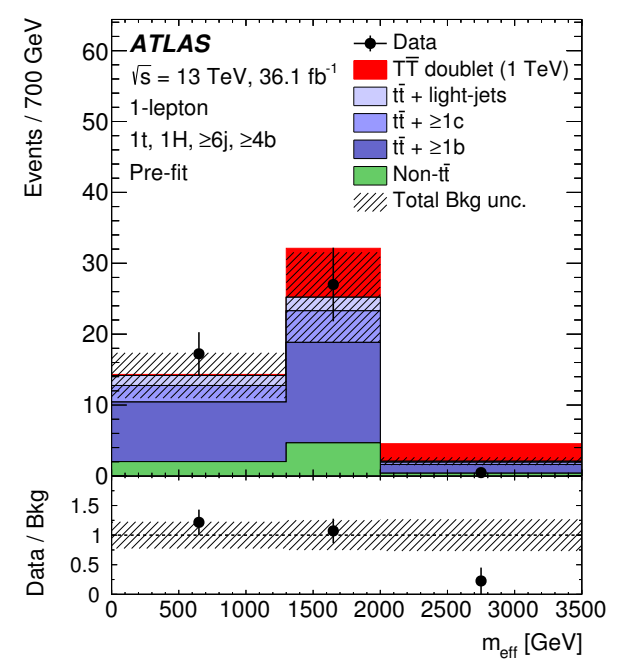

(a)

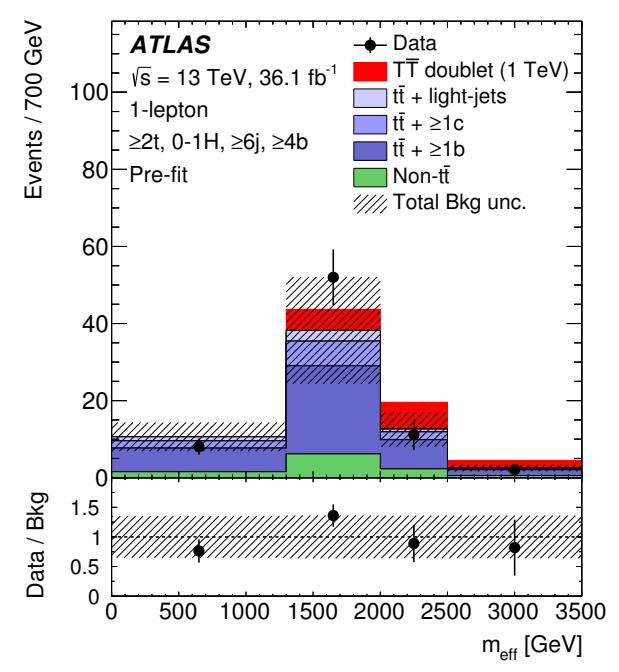

(c)

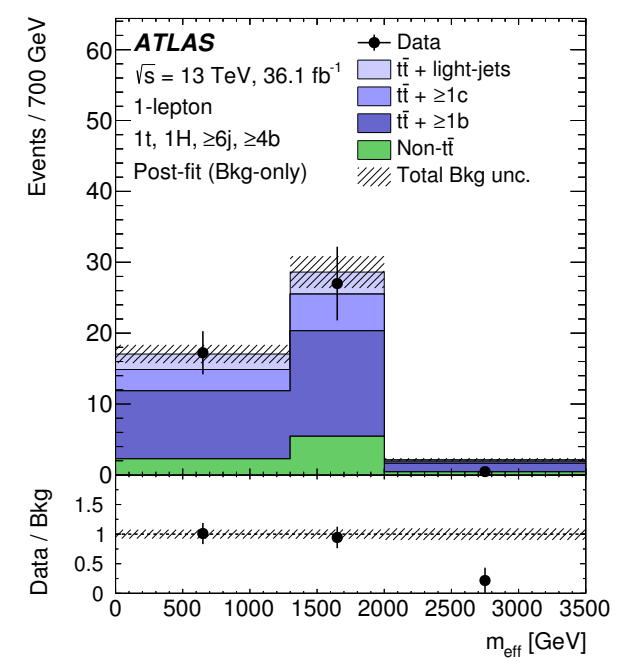

(b)

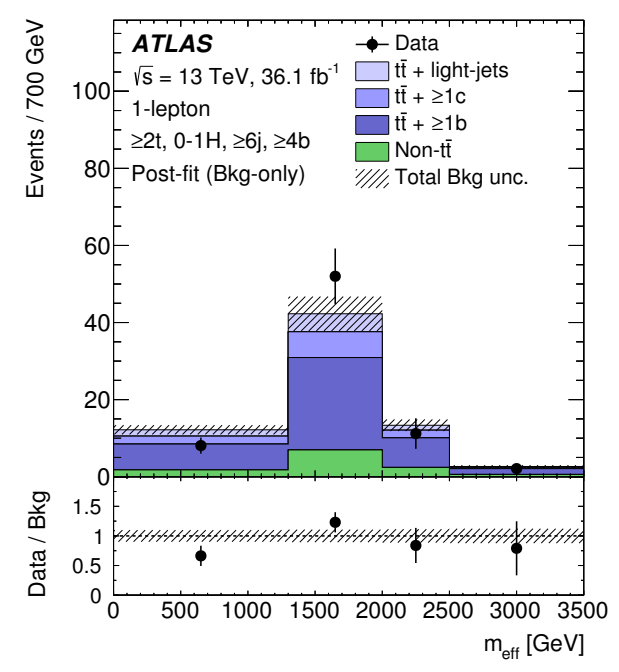

(d)

Figure 10. Comparison between the data and prediction for the $m_{\text {eff }}$ distribution in some of the most sensitive search regions in the 1-lepton channel, before and after performing the combined fit to data in the 0-lepton and 1-lepton channels ("Pre-fit" and "Post-fit", respectively) under the background-only hypothesis. Shown are the $(1 \mathrm{t}, 1 \mathrm{H}, \geq 6 \mathrm{j}, \geq 4 \mathrm{~b})$ region (a) pre-fit and (b) post-fit, and the $(\geq 2 \mathrm{t}, 0-1 \mathrm{H}, \geq 6 \mathrm{j}, \geq 4 \mathrm{~b})$ region (c) pre-fit and (d) post-fit. In the pre-fit figures the expected $T \bar{T}$ signal (solid red) corresponding to $m_{T}=1 \mathrm{TeV}$ in the $T$ doublet scenario is also shown, added on top of the background prediction. The small contributions from $t \bar{t} V, t \bar{t} H$, single-top, $W / Z+$ jets, diboson, and multijet backgrounds are combined into a single background source referred to as "Non- $t \bar{t}$ ". The last bin in all figures contains the overflow. The bottom panels display the ratios of data to the total background prediction ("Bkg"). The blue triangles indicate points that are outside the vertical range of the figure. The hashed area represents the total uncertainty of the background. In the case of the pre-fit background uncertainty, the normalisation uncertainty of the $t \bar{t}+\geq 1 b$ background is not included. 


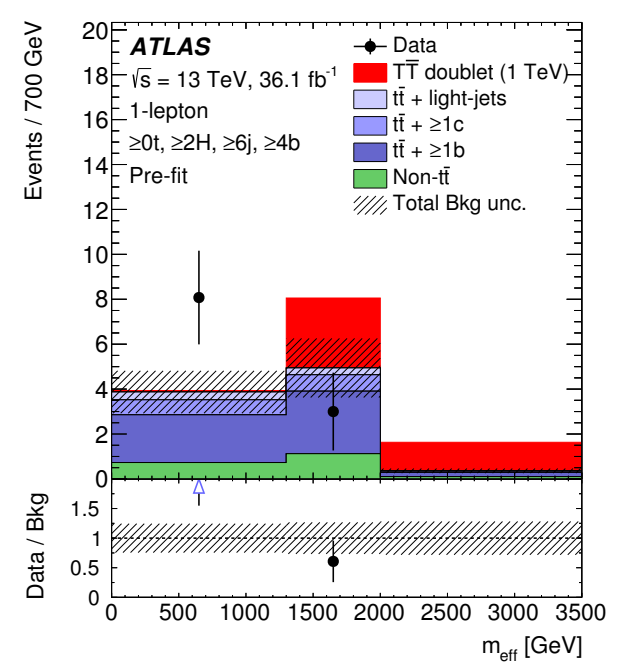

(a)

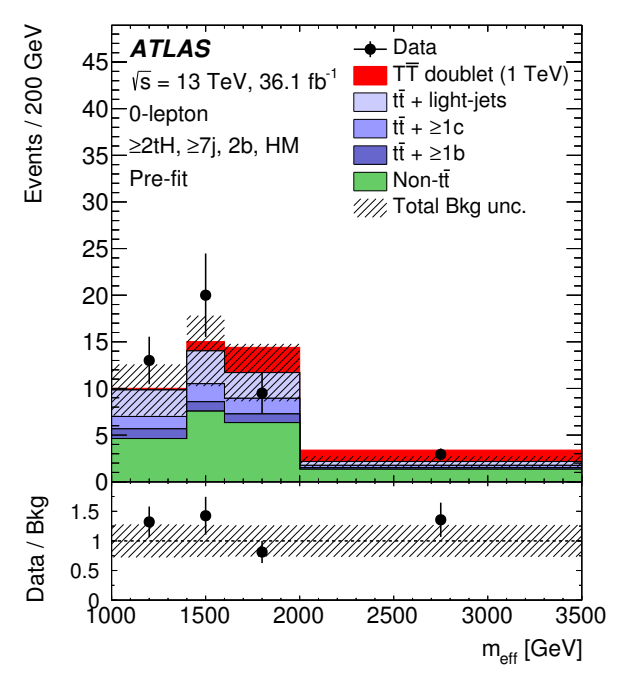

(c)

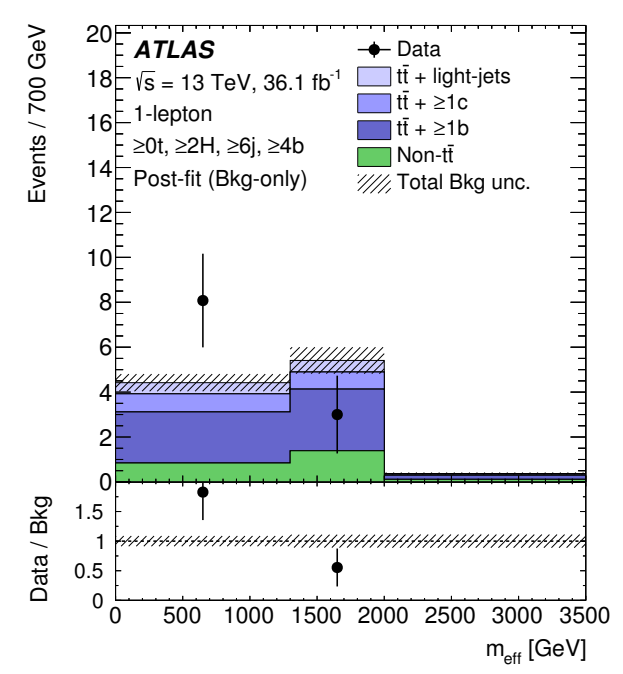

(b)

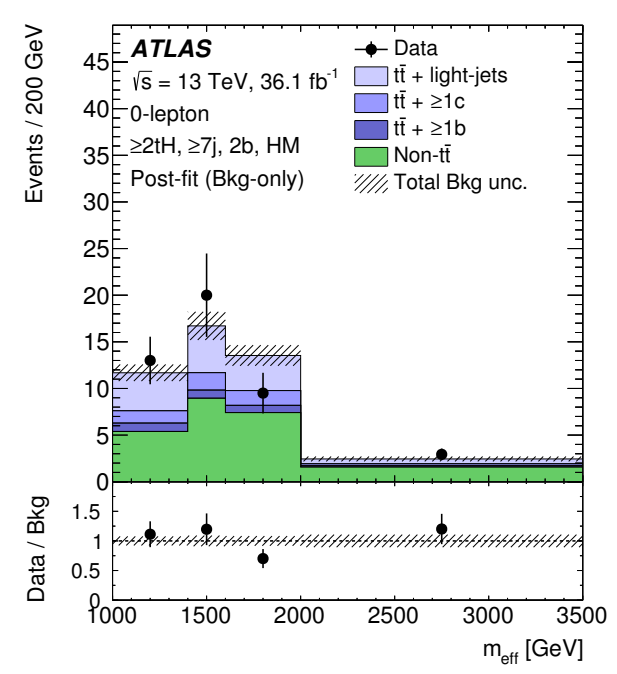

(d)

Figure 11. Comparison between the data and prediction for the $m_{\mathrm{eff}}$ distribution in some of the most sensitive search regions, before and after performing the combined fit to data in the 0-lepton and 1-lepton channels ("Pre-fit" and "Post-fit", respectively) under the background-only hypothesis. Shown are the $(\geq 2 \mathrm{H}, \geq 6 \mathrm{j}, \geq 4 \mathrm{~b})$ region in the 1-lepton channel (a) pre-fit and (b) post-fit, and the $(\geq 2 \mathrm{tH}, \geq 7 \mathrm{j}, 2 \mathrm{~b}, \mathrm{HM})$ region in the 0-lepton channel (c) pre-fit and (d) post-fit. In the pre-fit figures the expected $T \bar{T}$ signal (solid red) corresponding to $m_{T}=1 \mathrm{TeV}$ in the $T$ doublet scenario is also shown, added on top of the background prediction. The small contributions from $t \bar{t} V, t \bar{t} H$, single top, $W / Z+$ jets, diboson, and multijet backgrounds are combined into a single background source referred to as "Non- $t \bar{t}$ ". The last bin in all figures contains the overflow. The bottom panels display the ratios of data to the total background prediction ("Bkg"). The blue triangles indicate points that are outside the vertical range of the figure. The hashed area represents the total uncertainty of the background. In the case of the pre-fit background uncertainty, the normalisation uncertainty of the $t \bar{t}+\geq 1 b$ background is not included. 


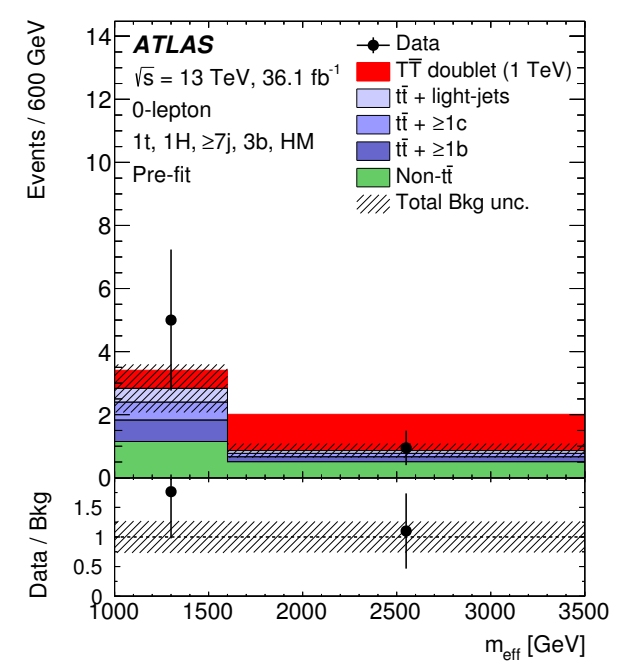

(a)

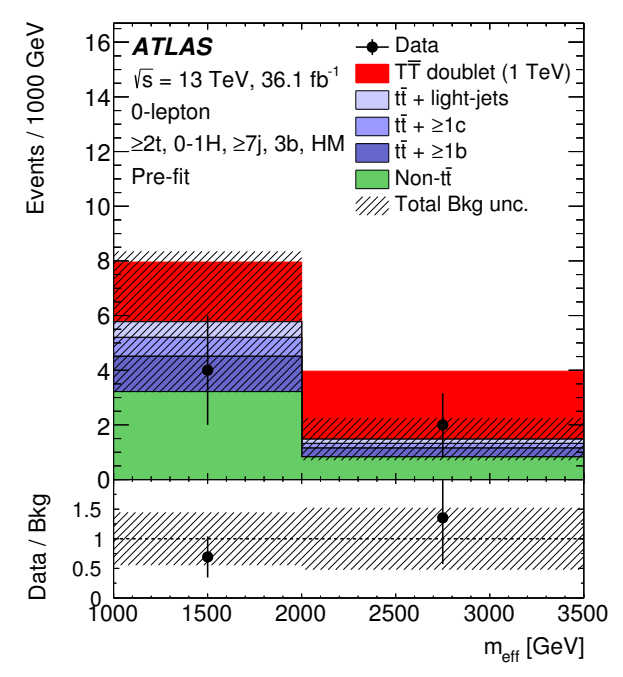

(c)

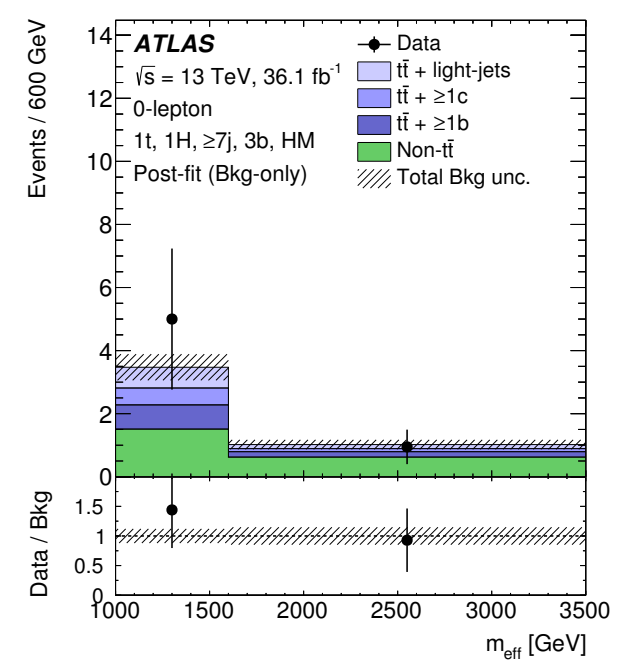

(b)

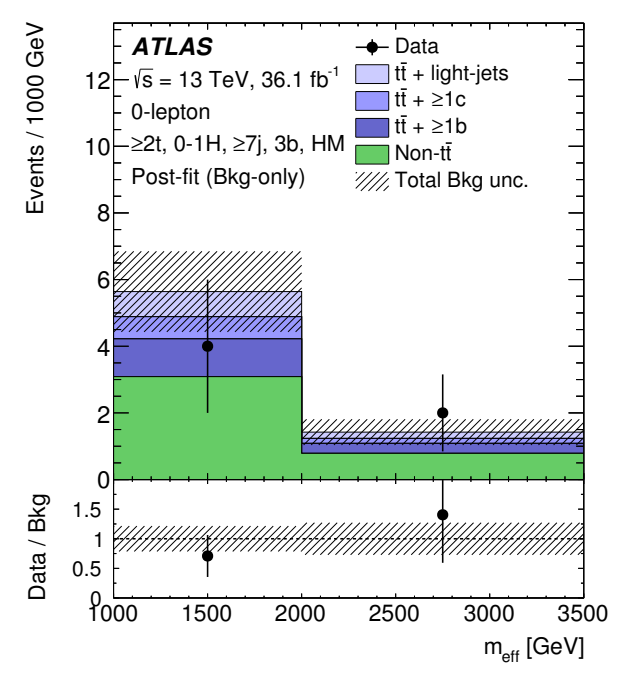

(d)

Figure 12. Comparison between the data and prediction for the $m_{\mathrm{eff}}$ distribution in some of the most sensitive search regions in the 0-lepton channel, before and after performing the combined fit to data in the 0-lepton and 1-lepton channels ("Pre-fit" and "Post-fit", respectively) under the background-only hypothesis. Shown are the $(1 \mathrm{t}, 1 \mathrm{H}, \geq 7 \mathrm{j}, 3 \mathrm{~b}, \mathrm{HM})$ region (a) pre-fit and (b) postfit, and the $(\geq 2 \mathrm{t}, 0-1 \mathrm{H}, \geq 7 \mathrm{j}, 3 \mathrm{~b}, \mathrm{HM})$ region (c) pre-fit and (d) post-fit. In the pre-fit figures the expected $T \bar{T}$ signal (solid red) corresponding to $m_{T}=1 \mathrm{TeV}$ in the $T$ doublet scenario is also shown, added on top of the background prediction. The small contributions from $t \bar{t} V, t \bar{t} H$, singletop, $W / Z+$ jets, diboson, and multijet backgrounds are combined into a single background source referred to as "Non- $t \bar{t}$ ". The last bin in all figures contains the overflow. The bottom panels display the ratios of data to the total background prediction ("Bkg"). The hashed area represents the total uncertainty of the background. In the case of the pre-fit background uncertainty, the normalisation uncertainty of the $t \bar{t}+\geq 1 b$ background is not included. 


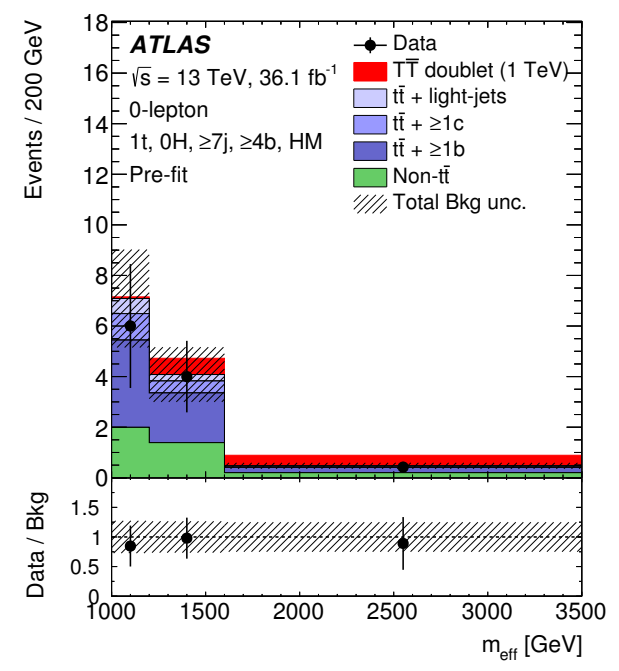

(a)

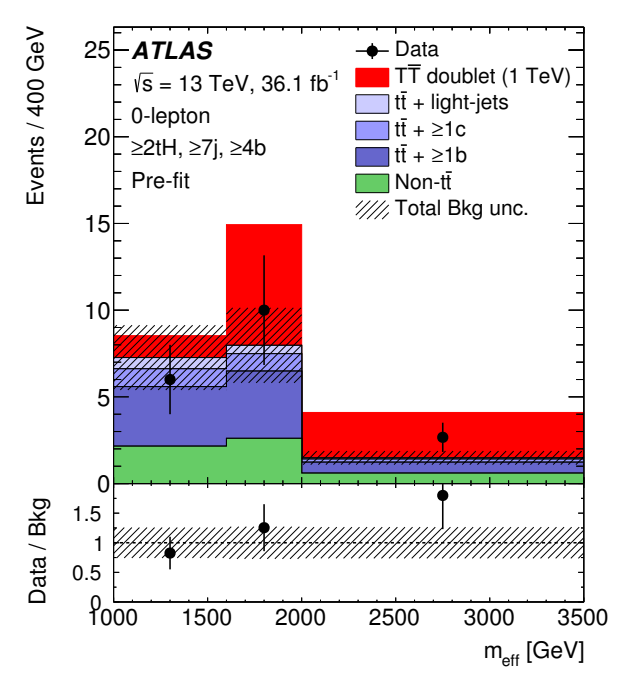

(c)

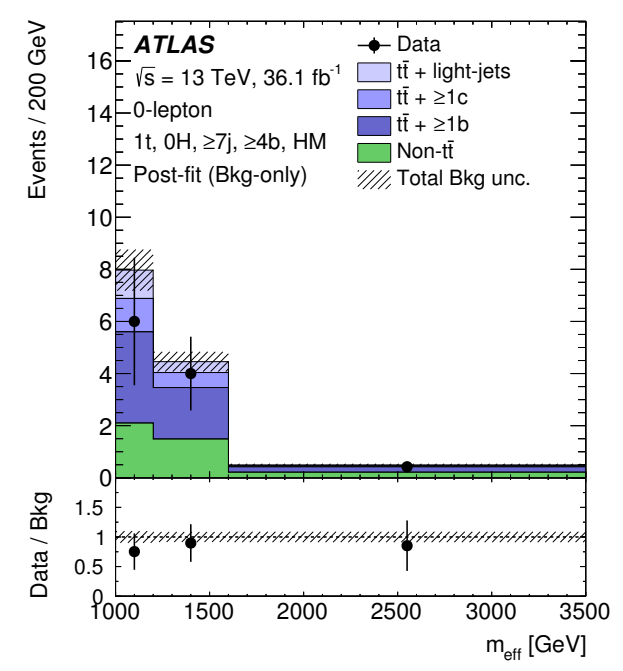

(b)

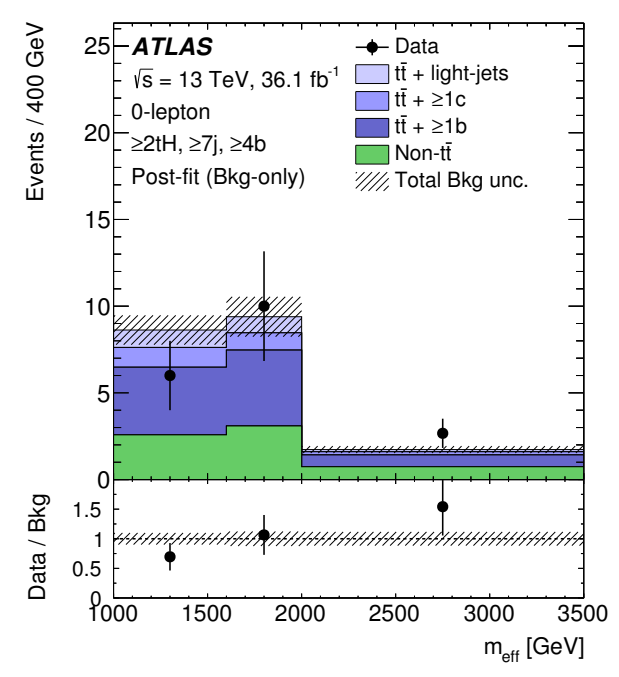

(d)

Figure 13. Comparison between the data and prediction for the $m_{\mathrm{eff}}$ distribution in some of the most sensitive search regions in the 0-lepton channel, before and after performing the combined fit to data in the 0-lepton and 1-lepton channels ("Pre-fit" and "Post-fit", respectively) under the background-only hypothesis. Shown are the $(1 \mathrm{t}, 0 \mathrm{H}, \geq 7 \mathrm{j}, \geq 4 \mathrm{~b}, \mathrm{HM})$ region (a) pre-fit and (b) postfit, and the $(\geq 2 \mathrm{tH}, \geq 7 \mathrm{j}, \geq 4 \mathrm{~b})$ region (c) pre-fit and (d) post-fit. In the pre-fit figures the expected $T \bar{T}$ signal (solid red) corresponding to $m_{T}=1 \mathrm{TeV}$ in the $T$ doublet scenario is also shown, added on top of the background prediction. The small contributions from $t \bar{t} V, t \bar{t} H$, single-top, $W / Z+$ jets, diboson, and multijet backgrounds are combined into a single background source referred to as "Non- $t \bar{t}$ ". The last bin in all figures contains the overflow. The bottom panels display the ratios of data to the total background prediction ("Bkg"). The hashed area represents the total uncertainty of the background. In the case of the pre-fit background uncertainty, the normalisation uncertainty of the $t \bar{t}+\geq 1 b$ background is not included. 


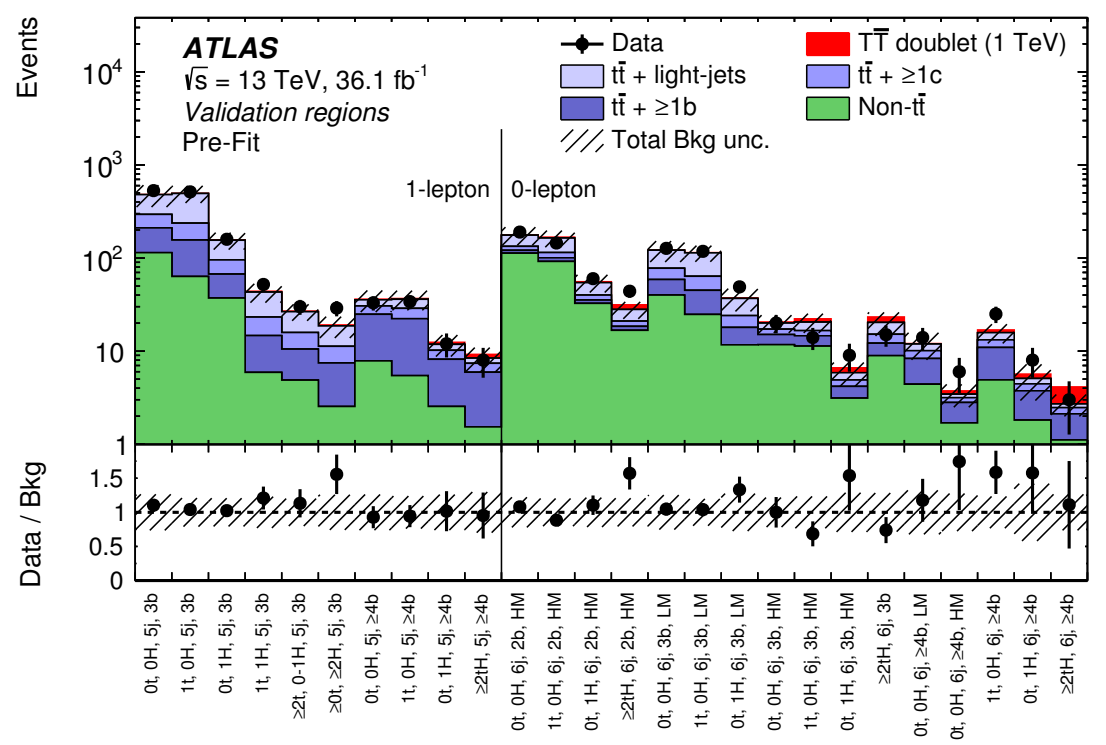

(a)

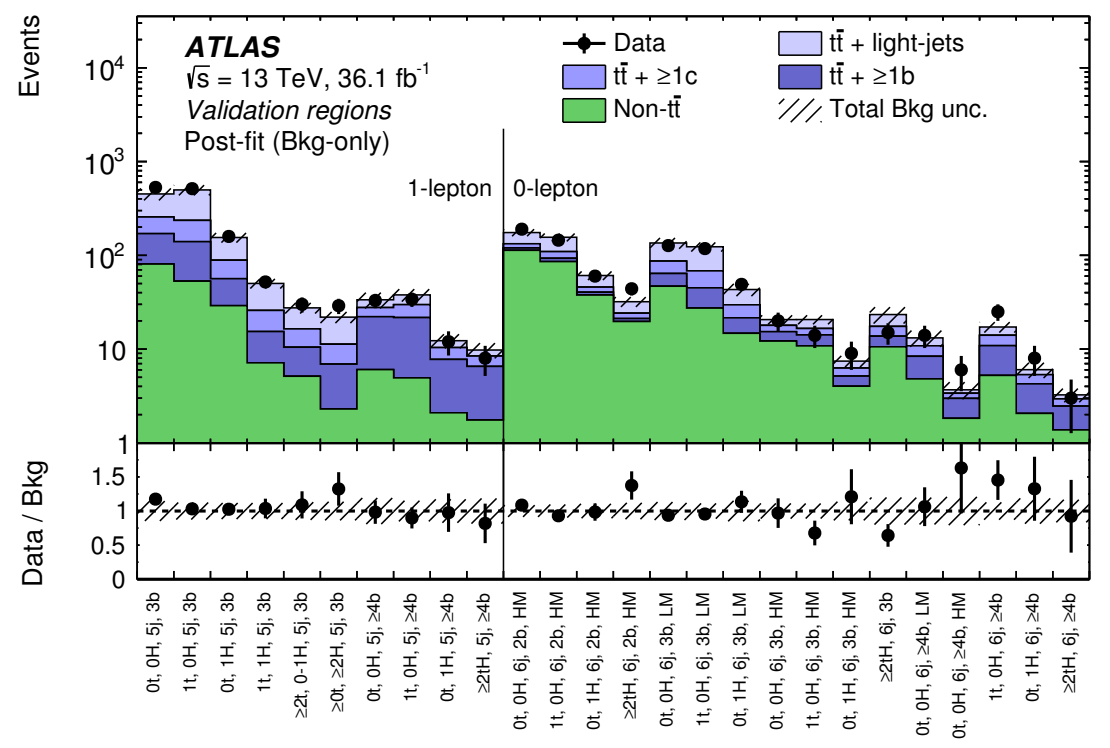

(b)

Figure 14. Comparison between the data and background prediction for the yields in each of the validation regions considered in the 1-lepton and 0-lepton channels (a) before the fit ("Pre-fit") and (b) after the fit ("Post-fit"). The fit is performed on the data in 1-lepton and 0-lepton channels under the background-only hypothesis considering only the search regions. In the pre-fit figure the expected $T \bar{T}$ signal (solid red) corresponding to $m_{T}=1 \mathrm{TeV}$ in the $T$ doublet scenario is also shown, added on top of the background prediction. The small contributions from $t \bar{t} V, t \bar{t} H$, single-top, $W / Z+$ jets, diboson, and multijet backgrounds are combined into a single background source referred to as "Non- $t \bar{t}$ ". The bottom panels display the ratios of data to the total background prediction ("Bkg"). The hashed area represents the total uncertainty of the background. In the case of the pre-fit background uncertainty, the normalisation uncertainty of the $t \bar{t}+\geq 1 b$ background is not included. 


\begin{tabular}{|lccccc|}
\hline 1-lepton channel & $\geq 2 \mathrm{t}, 0-1 \mathrm{H}$, & $1 \mathrm{t}, 0 \mathrm{H}$, & $1 \mathrm{t}, 1 \mathrm{H}$, & $\geq 2 \mathrm{t}, 0-1 \mathrm{H}$, & $\geq 0 \mathrm{t}, \geq 2 \mathrm{H}$, \\
& $\geq 6 \mathrm{j}, 3 \mathrm{~b}$ & $\geq 6 \mathrm{j}, \geq 4 \mathrm{~b}$ & $\geq 6 \mathrm{j}, \geq 4 \mathrm{~b}$ & $\geq 6 \mathrm{j}, \geq 4 \mathrm{~b}$ & $\geq 6 \mathrm{j}, \geq 4 \mathrm{~b}$ \\
\hline$t \bar{t}+$ light-jets & $137 \pm 24$ & $59 \pm 11$ & $7.6 \pm 1.6$ & $9.0 \pm 2.0$ & $1.50 \pm 0.34$ \\
$t \bar{t}+\geq 1 c$ & $79 \pm 34$ & $81 \pm 26$ & $11.4 \pm 3.8$ & $12.4 \pm 5.1$ & $2.36 \pm 0.84$ \\
$t \bar{t}+\geq 1 b$ & $84 \pm 20$ & $217 \pm 27$ & $35.3 \pm 5.6$ & $44.1 \pm 9.1$ & $7.4 \pm 1.2$ \\
$t \bar{t} V$ & $10.7 \pm 1.6$ & $13.2 \pm 2.1$ & $2.12 \pm 0.34$ & $2.82 \pm 0.46$ & $0.50 \pm 0.08$ \\
$t \bar{t} H$ & $5.26 \pm 0.61$ & $17.4 \pm 2.3$ & $4.28 \pm 0.56$ & $3.25 \pm 0.46$ & $1.33 \pm 0.17$ \\
$W+$ jets & $11.4 \pm 4.0$ & $9.5 \pm 3.4$ & $0.71 \pm 0.36$ & $1.68 \pm 0.59$ & $0.78 \pm 0.31$ \\
$Z+$ jets & $1.56 \pm 0.55$ & $1.11 \pm 0.41$ & $0.08 \pm 0.06$ & $0.16 \pm 0.06$ & $0.07 \pm 0.04$ \\
Single top & $11.3 \pm 5.6$ & $10.8 \pm 6.2$ & $2.01 \pm 0.62$ & $1.85 \pm 0.90$ & $0.24 \pm 0.15$ \\
Diboson & $2.20 \pm 0.91$ & $1.10 \pm 0.50$ & $0.20 \pm 0.08$ & $0.30 \pm 0.12$ & $0.03 \pm 0.07$ \\
$t \bar{t} t \bar{t}$ (SM) & $2.83 \pm 0.84$ & $5.3 \pm 1.5$ & $1.20 \pm 0.35$ & $2.74 \pm 0.79$ & $0.24 \pm 0.07$ \\
\hline Total background & $349 \pm 20$ & $416 \pm 18$ & $64.9 \pm 4.7$ & $78.2 \pm 8.0$ & $14.4 \pm 1.2$ \\
\hline Data & 353 & 428 & 60 & 78 & 18 \\
\hline
\end{tabular}

Table 6. Predicted and observed yields in the 1-lepton channel in five of the most sensitive search regions (depending on the signal scenario) considered. The multijet background is considered negligible in these regions and thus not shown. The background prediction is shown after the combined fit to data in the 0-lepton and 1-lepton channels under the background-only hypothesis. The quoted uncertainties are the sum in quadrature of statistical and systematic uncertainties in the yields, computed taking into account correlations among nuisance parameters and among processes.

theoretical cross section. The scenarios considered involve different assumptions about the decay branching ratios. The search in the 1-lepton (0-lepton) channel is particularly sensitive to the benchmark scenario of $\mathcal{B}(T \rightarrow H t)=1(\mathcal{B}(T \rightarrow Z t)=1)$. In contrast, both the 1-lepton and the 0-lepton searches have comparable sensitivity to the weak-isospin doublet and singlet scenarios, and thus their combination represents an improvement of 60 $70 \mathrm{GeV}$ on the expected $T$ quark mass exclusion over the most sensitive individual search. The limits corresponding to the weak-isospin doublet and singlet scenarios obtained for the combination of the 1-lepton and 0-lepton searches are shown in figure 17. A summary of the observed and expected lower limits on the $T$ quark mass in the different benchmark scenarios for the individual 1-lepton and 0-lepton searches, as well as their combination, is given in table 8. As can be seen, the observed mass limits for the 1-lepton search are above the expected limits in all benchmark scenarios. Detailed studies on the statistical model found no sources of systematic bias and showed that the results are consistent with downward statistical fluctuations in data in some of the highest $m_{\mathrm{eff}}$ bins in three search regions: (1t, $1 \mathrm{H}, \geq 6 \mathrm{j}, \geq 4 \mathrm{~b}),(\geq 2 \mathrm{t}, 0-1 \mathrm{H}, \geq 6 \mathrm{j}, 3 \mathrm{~b})$, and $(\geq 0 \mathrm{t}, \geq 2 \mathrm{H}, \geq 6 \mathrm{j}, \geq 4 \mathrm{~b})$. Several other regions with similar event kinematics and background composition to these three search regions show good agreement between data and expectations. In particular, additional 


\begin{tabular}{|lccccc|}
\hline 0-lepton channel & $\begin{array}{c}\geq \mathrm{tH}, \\
\geq 7 \mathrm{j}, 2 \mathrm{~b}, \mathrm{HM}\end{array}$ & $\begin{array}{c}1 \mathrm{t}, 1 \mathrm{H}, \\
\geq \mathrm{j}, 3 \mathrm{~b}, \mathrm{HM}\end{array}$ & $\begin{array}{c}\geq 2 \mathrm{t}, 0-1 \mathrm{H}, \\
\geq 7 \mathrm{j}, 3 \mathrm{~b}, \mathrm{HM}\end{array}$ & $\begin{array}{c}1 \mathrm{t}, 0 \mathrm{H}, \\
\text { t }, \geq 4 \mathrm{~b}, \mathrm{HM}\end{array}$ & $\begin{array}{c}\geq 2 \mathrm{tH}, \\
\geq \mathrm{j}, \geq 4 \mathrm{~b}\end{array}$ \\
\hline$t \bar{t}+$ light-jets & $24.7 \pm 5.0$ & $1.08 \pm 0.20$ & $1.04 \pm 0.25$ & $2.20 \pm 0.43$ & $2.91 \pm 0.57$ \\
$t \bar{t}+\geq 1 c$ & $9.2 \pm 4.9$ & $0.85 \pm 0.44$ & $0.89 \pm 0.48$ & $2.9 \pm 1.1$ & $3.4 \pm 1.4$ \\
$t \bar{t}+\geq 1 b$ & $5.3 \pm 1.9$ & $1.31 \pm 0.39$ & $1.58 \pm 0.55$ & $9.4 \pm 1.3$ & $12.8 \pm 2.4$ \\
$t \bar{t} V$ & $5.96 \pm 0.88$ & $0.59 \pm 0.09$ & $1.00 \pm 0.15$ & $1.46 \pm 0.23$ & $1.25 \pm 0.19$ \\
$t \bar{t} H$ & $0.61 \pm 0.08$ & $0.19 \pm 0.03$ & $0.13 \pm 0.02$ & $1.02 \pm 0.13$ & $1.16 \pm 0.17$ \\
$W+$ jets & $12.0 \pm 3.2$ & $0.63 \pm 0.22$ & $0.92 \pm 0.34$ & $0.71 \pm 0.27$ & $0.86 \pm 0.22$ \\
$Z+$ jets & $10.6 \pm 3.1$ & $0.69 \pm 0.26$ & $0.4 \pm 1.3$ & $0.65 \pm 0.29$ & $0.94 \pm 0.29$ \\
Single top & $8.9 \pm 3.2$ & $0.77 \pm 0.36$ & $0.95 \pm 0.48$ & $1.84 \pm 0.82$ & $1.17 \pm 0.47$ \\
Diboson & $3.9 \pm 1.6$ & $0.41 \pm 0.39$ & $0.53 \pm 0.44$ & $0.37 \pm 0.15$ & $0.23 \pm 0.10$ \\
$t \bar{t} t \bar{t}$ (SM) & $0.20 \pm 0.07$ & $0.05 \pm 0.02$ & $0.12 \pm 0.04$ & $0.36 \pm 0.10$ & $0.87 \pm 0.24$ \\
Multijet & $4.1 \pm 3.7$ & $0.14 \pm 0.13$ & $0.18 \pm 0.19$ & $0.67 \pm 0.62$ & $3.3 \pm 2.6$ \\
\hline Total background & $85.5 \pm 6.8$ & $6.70 \pm 0.75$ & $7.8 \pm 1.7$ & $21.6 \pm 1.4$ & $28.8 \pm 3.1$ \\
\hline Data & 87 & 8 & 7 & 18 & 29 \\
\hline
\end{tabular}

Table 7. Predicted and observed yields in the 0-lepton channel in five of the most sensitive search regions (depending on the signal scenario) considered. The background prediction is shown after the combined fit to data in the 0-lepton and 1-lepton channels under the background-only hypothesis. The quoted uncertainties are the sum in quadrature of statistical and systematic uncertainties in the yields, computed taking into account correlations among nuisance parameters and among processes.

regions with larger event yields were constructed to test this agreement by merging signal regions in certain categories, but retaining similar multiplicities of b-tagged jets or boosted objects as the original signal regions.

Table 8 also includes a comparison to the limits obtained by the ATLAS Run- $T \bar{T} \rightarrow$ $H t+\mathrm{X}$ search in the 1-lepton channel [25]: the current results extend the expected $T$ quark mass exclusion by $\sim 390-490 \mathrm{GeV}$, depending on the assumed benchmark scenario.

The same analyses are used to derive exclusion limits on vector-like $T$ quark production, for different values of $m_{T}$ and as a function of $\mathcal{B}(T \rightarrow W b)$ and $\mathcal{B}(T \rightarrow H t)$, assuming that $\mathcal{B}(T \rightarrow W b)+\mathcal{B}(T \rightarrow Z t)+\mathcal{B}(T \rightarrow H t)=1$. To probe this branching ratio plane, the signal samples are reweighted by the ratio of the desired branching ratio to the original branching ratio in PROTOs, and the complete analysis is repeated. Owing to the complementarity of the 1-lepton and 0-lepton searches in probing the branching ratio plane, their combination represents a significant improvement over the individual results, as illustrated in figure 18. In this case, the observed lower limits on the $T$ quark mass range between $0.99 \mathrm{TeV}$ and $1.43 \mathrm{TeV}$ depending on the values of the branching ratios into the three decay modes. In particular, a vector-like $T$ quark with mass below $0.99 \mathrm{TeV}$ is excluded for any values of the branching ratios into the three decay modes. The corresponding range of expected lower limits is between $0.91 \mathrm{TeV}$ and $1.34 \mathrm{TeV}$. Figure 19 presents the corresponding observed and expected $T$ quark mass limits in the plane of $\mathcal{B}(T \rightarrow H t)$ versus $\mathcal{B}(T \rightarrow W b)$, obtained by linear interpolation of the calculated $\mathrm{CL}_{\mathrm{s}}$ versus $m_{T}$. 


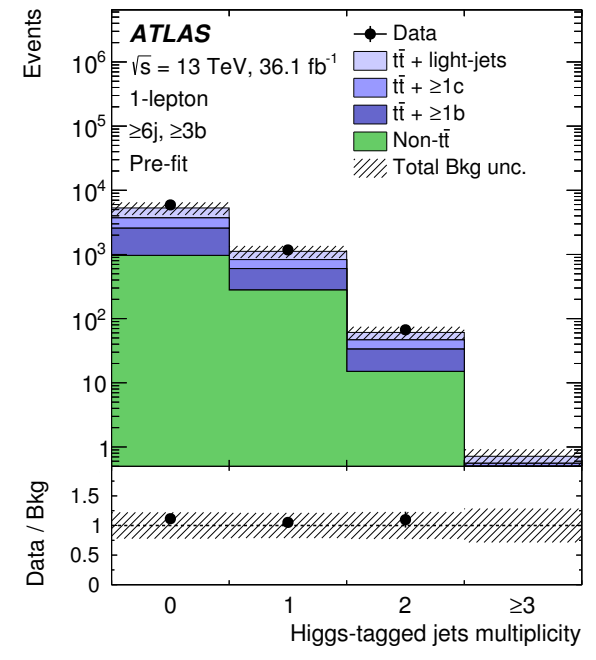

(a)

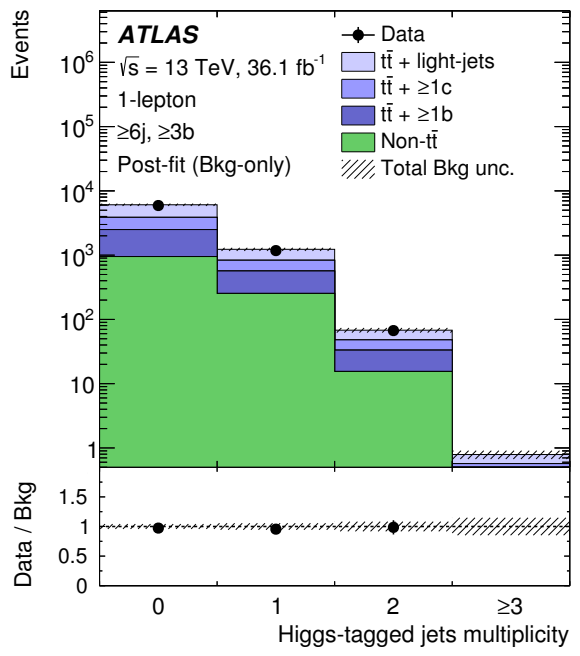

(b)

Figure 15. Comparison between the data and prediction for the Higgs-tagged jet multiplicity in the 1-lepton channel after preselection plus the requirement of $\geq 6$ jets and $\geq 3$-tagged jets, (a) before and (b) after performing the combined fit of the $m_{\text {eff }}$ spectrum to data in the 0-lepton and 1-lepton channels search regions ("Pre-fit" and "Post-fit", respectively) under the background-only hypothesis. The small contributions from $t \bar{t} V, t \bar{t} H$, single-top, $W / Z+$ jets, diboson, and multijet backgrounds are combined into a single background source referred to as "Non- $t \bar{t}$ ". The last bin in all figures contains the overflow. The bottom panels display the ratios of data to the total background prediction ("Bkg"). The hashed area represents the total uncertainty of the background. In the case of the pre-fit background uncertainty, the normalisation uncertainty of the $t \bar{t}+\geq 1 b$ background is not included.

\begin{tabular}{|lcccc|}
\hline \multicolumn{5}{|c|}{$95 \%$ CL lower limits on $T$ quark mass $[\mathrm{TeV}]$} \\
\hline Search & $\mathcal{B}(T \rightarrow H t)=1$ & $\mathcal{B}(T \rightarrow Z t)=1$ & Doublet & Singlet \\
\hline 1-lepton channel & $1.47(1.30)$ & $1.12(0.91)$ & $1.36(1.16)$ & $1.23(1.02)$ \\
0-lepton channel & $1.11(1.20)$ & $1.12(1.17)$ & $1.12(1.19)$ & $0.99(1.05)$ \\
\hline Combination & $1.43(1.34)$ & $1.17(1.18)$ & $1.31(1.26)$ & $1.19(1.11)$ \\
\hline \multicolumn{5}{|c}{} \\
\hline Previous Run-1 ATLAS $T \bar{T} \rightarrow H t+\mathrm{X}$ search $[25]$ \\
\hline 1-lepton channel & $0.95(0.88)$ & $0.75(0.69)$ & $0.86(0.82)$ & $0.76(0.72)$ \\
\hline
\end{tabular}

Table 8. Summary of observed (expected) 95\% CL lower limits on $T$ quark mass (in TeV) for the 1-lepton and 0-lepton channels, as well as their combination, with different assumptions about the decay branching ratios. The background estimate used in the computation of the limits is the result obtained from the background-only fit to data. Also shown are the corresponding limits obtained by the Run-1 ATLAS $T \bar{T} \rightarrow H t+\mathrm{X}$ search in the 1-lepton channel [25]. 


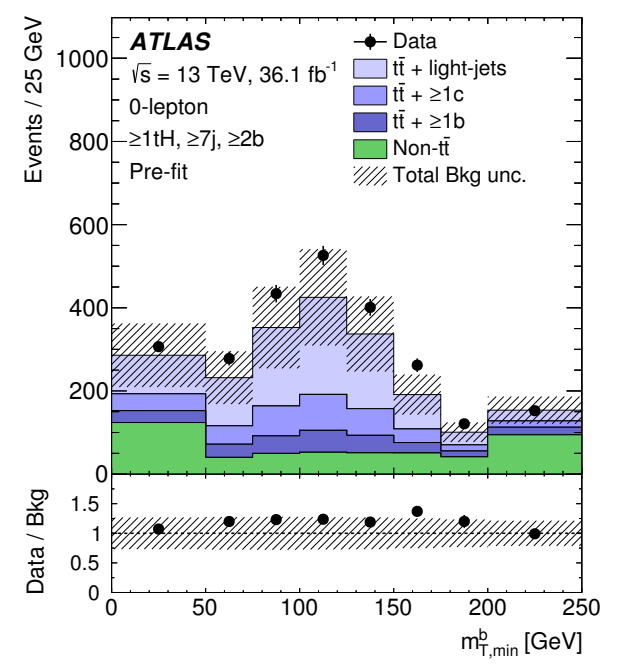

(a)

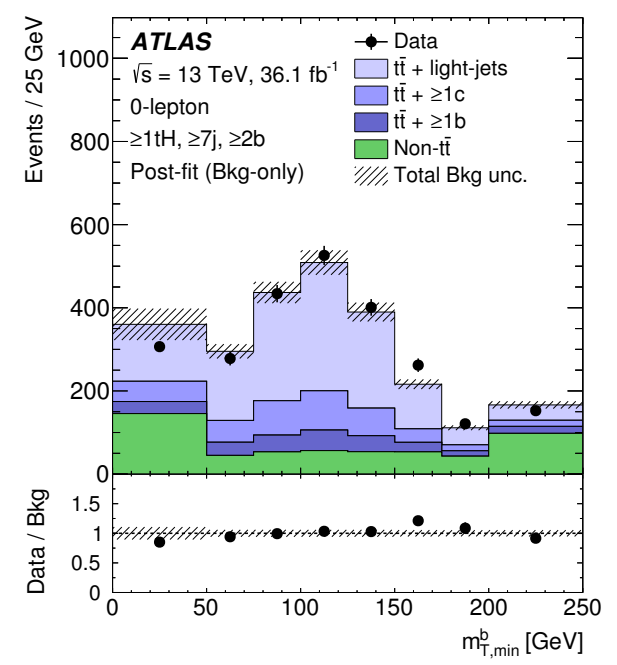

(b)

Figure 16. Comparison between the data and prediction for the distribution of the minimum transverse mass of $E_{\mathrm{T}}^{\mathrm{miss}}$ and any of the three leading $b$-tagged jets in the event $\left(m_{\mathrm{T}, \min }^{\mathrm{b}}\right)$ in the $(\geq 1 \mathrm{tH}, \geq 7 \mathrm{j}, \geq 2 \mathrm{~b}$ ) region of the 0-lepton channel (a) before and (b) after performing the combined fit of the $m_{\mathrm{eff}}$ spectrum to data in the 0-lepton and 1-lepton channels search regions ("Pre-fit" and "Post-fit", respectively) under the background-only hypothesis. The small contributions from $t \bar{t} V, t \bar{t} H$, single-top, $W / Z+$ jets, diboson, and multijet backgrounds are combined into a single background source referred to as "Non- $t \bar{t}$ ". The last bin in all figures contains the overflow. The bottom panels display the ratios of data to the total background prediction ("Bkg"). The hashed area represents the total uncertainty of the background. In the case of the pre-fit background uncertainty, the normalisation uncertainty of the $t \bar{t}+\geq 1 b$ background is not included.

\subsection{Limits on four-top-quark production}

The 1-lepton search is used to set limits on BSM four-top-quark production by considering different signal benchmark scenarios (see section 5.1 for details). In the case of $t \bar{t} t \bar{t}$ production via an EFT model with a four-top-quark contact interaction, the observed (expected) $95 \%$ CL upper limit on the production cross section is $16 \mathrm{fb}\left(31_{-9}^{+12} \mathrm{fb}\right)$. The upper limit on the production cross section can be translated into an observed (expected) limit on the free parameter of the model $\left|C_{4 t}\right| / \Lambda^{2}<1.6 \mathrm{TeV}^{-2}\left(2.3 \pm 0.4 \mathrm{TeV}^{-2}\right)$. In the context of the 2UED/RPP model, the observed and expected upper limits on the production cross section times branching ratio are shown in figure 20 as a function of $m_{\mathrm{KK}}$ for the symmetric case $\left(\xi=R_{4} / R_{5}=1\right)$, assuming production by tier $(1,1)$ alone. The comparison to the LO theoretical cross section translates into an observed (expected) $95 \%$ CL limit on $m_{K K}$ of $1.8 \mathrm{TeV}(1.7 \mathrm{TeV})$. 


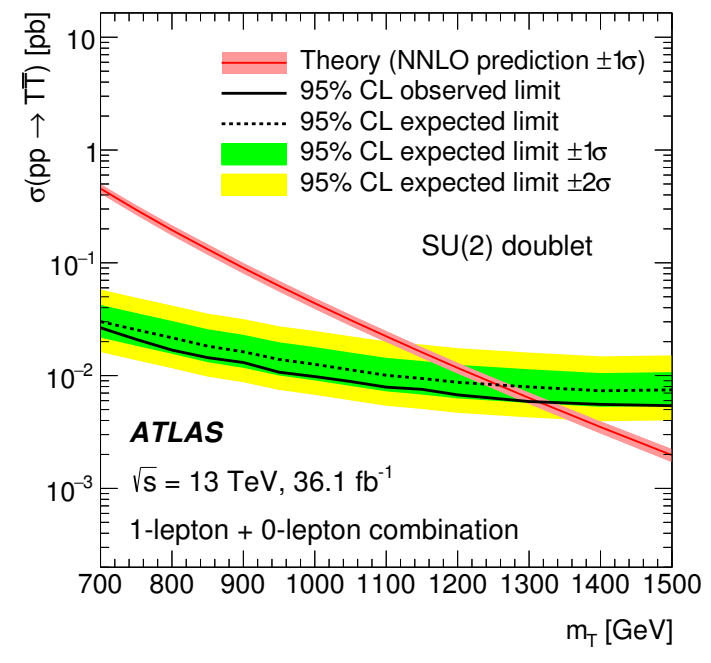

(a)

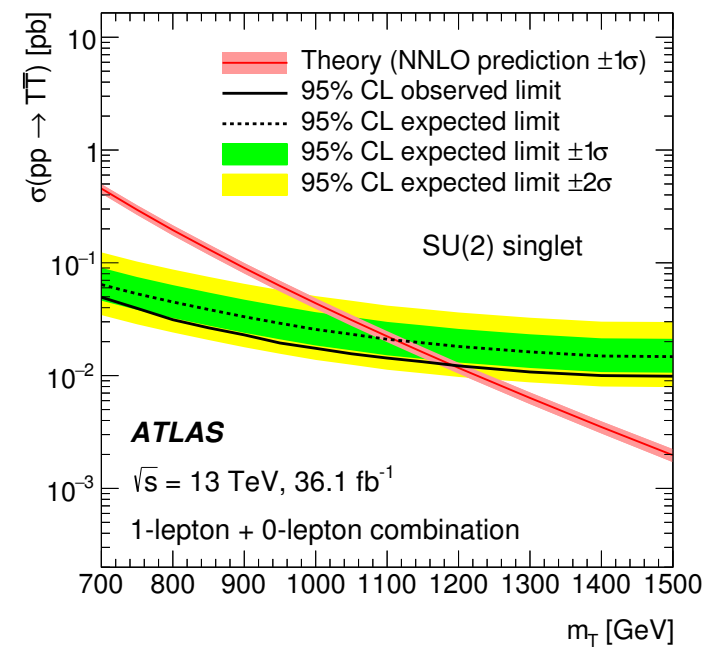

(b)

Figure 17. Observed (solid line) and expected (dashed line) 95\% CL upper limits on the $T \bar{T}$ cross section as a function of the $T$ quark mass for the combination of the 1-lepton and 0-lepton searches (a) for a $T$ quark doublet, and (b) for a $T$ quark singlet. The background estimate used in the computation of the limits is the result obtained from the background-only fit to data. The surrounding shaded bands correspond to \pm 1 and \pm 2 standard deviations around the expected limit. The thin red line and band show the theoretical prediction and its \pm 1 standard deviation uncertainty. 


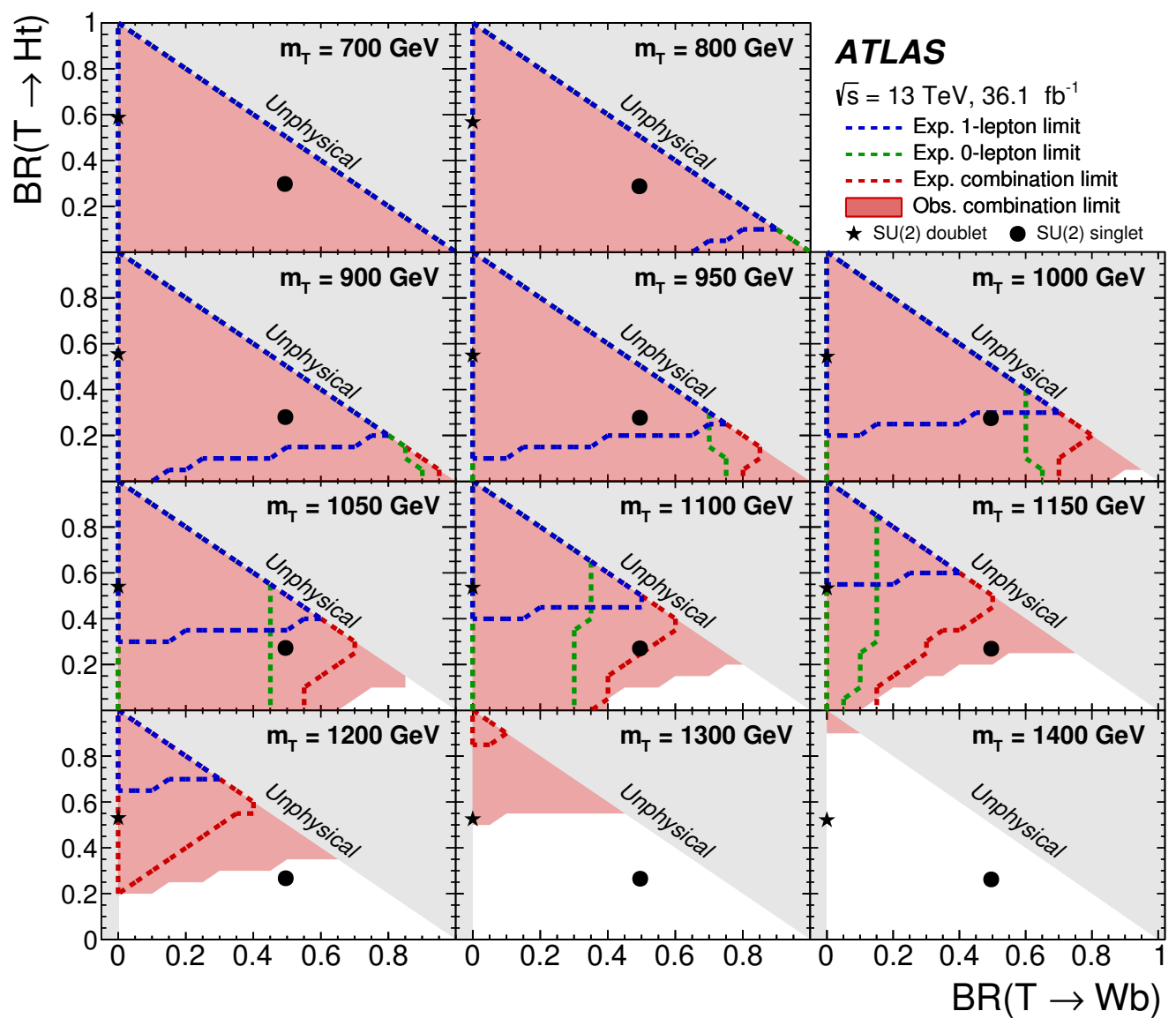

Figure 18. Observed (red filled area) and expected (red dashed line) 95\% CL exclusion in the plane of $\mathcal{B}(T \rightarrow W b)$ versus $\mathcal{B}(T \rightarrow H t)$, for different values of the vector-like $T$ quark mass for the combination of the 1-lepton and 0-lepton searches. In the figure, the branching ratio is denoted "BR". The background estimate used in the computation of the limits is the result obtained from the background-only fit to data. Also shown are the expected exclusions by the individual searches, which can be compared to that obtained through their combination. The grey (light shaded) area corresponds to the unphysical region where the sum of branching ratios exceeds unity, or is smaller than zero. The default branching ratio values from the PROTOs event generator for the weak-isospin singlet and doublet cases are shown as plain circle and star symbols, respectively. 


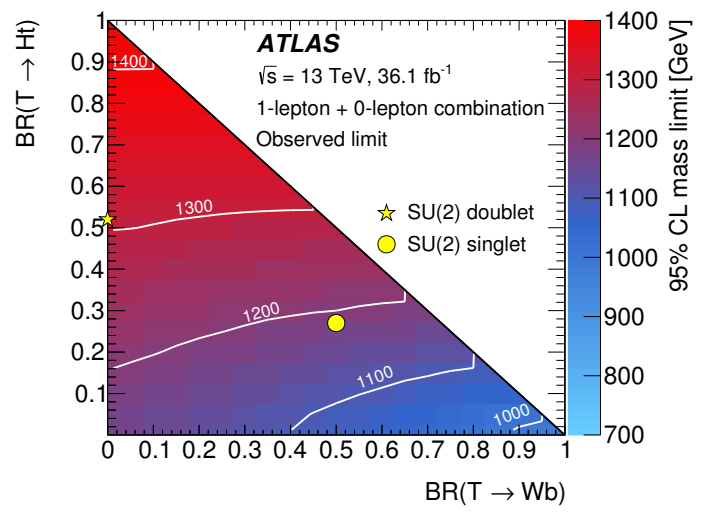

(a)

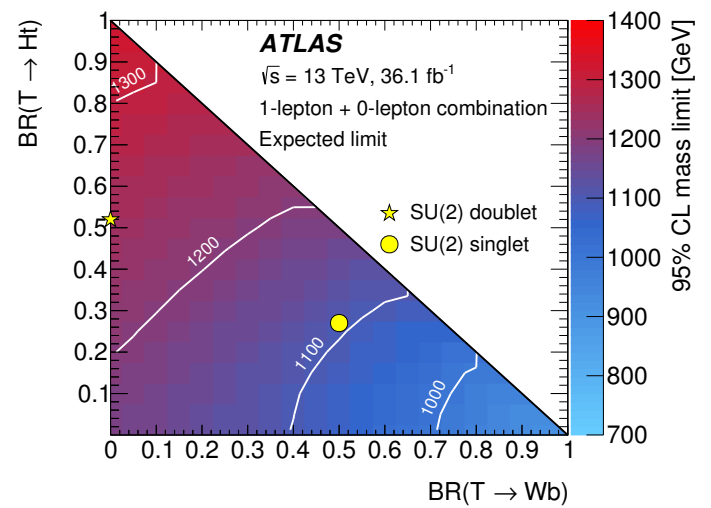

(b)

Figure 19. (a) Observed and (b) expected limit (95\% CL) on the mass of the $T$ quark in the plane of $\mathcal{B}(T \rightarrow H t)$ versus $\mathcal{B}(T \rightarrow W b)$ for the combination of the 1-lepton and 0-lepton searches. In the figure, the branching ratio is denoted "BR". The background estimate used in the computation of the limits is the result obtained from the background-only fit to data. Contour lines are provided to guide the eye. The yellow markers indicate the branching ratios for the $\mathrm{SU}(2)$ singlet and doublet scenarios with masses above $\approx 800 \mathrm{GeV}$, where they are approximately independent of the $T$ quark mass.

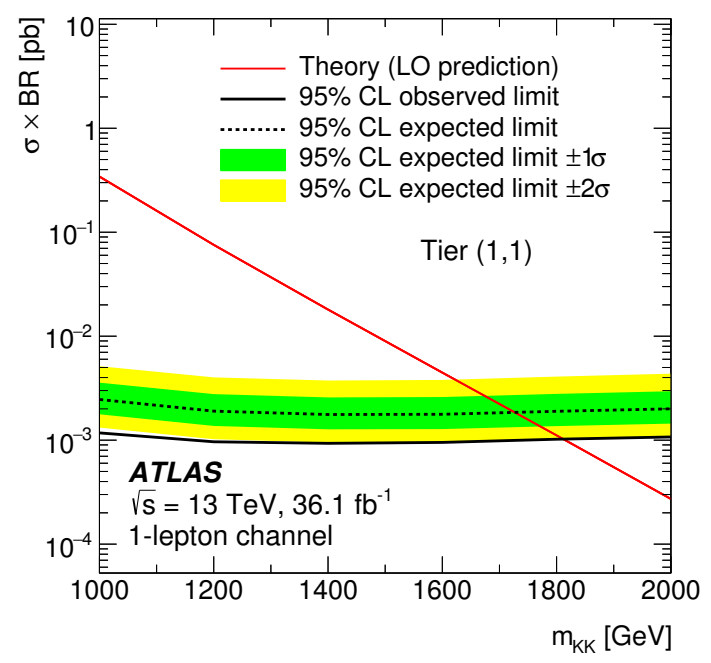

Figure 20. Observed (solid line) and expected (dashed line) 95\% CL upper limits on the production cross section times branching ratio of four-top-quark events as a function of the Kaluza-Klein mass $\left(m_{\mathrm{KK}}\right)$ from tier $(1,1)$ in the symmetric case $\left(\xi=R_{4} / R_{5}=1\right)$. The background estimate used in the computation of the limits is the result obtained from the background-only fit to data. The surrounding shaded bands correspond to \pm 1 and \pm 2 standard deviations around the expected limit. The thin red line shows the theoretical prediction, computed at LO in QCD, for the production cross section of four-top-quark events by tier $(1,1)$ assuming $\mathcal{B}\left(A^{(1,1)} \rightarrow t \bar{t}\right)=1$, where the heavy photon $A^{(1,1)}$ is the lightest particle of this tier. 


\section{Conclusion}

A search for pair production of up-type vector-like quarks $(T)$ with significant branching ratio into a top quark and either a Standard Model Higgs boson or a $Z$ boson is presented. The same analysis is also used to search for four-top-quark production, in several new physics scenarios. The search is based on $p p$ collisions at $\sqrt{s}=13 \mathrm{TeV}$ recorded in 2015 and 2016 with the ATLAS detector at the CERN Large Hadron Collider and corresponds to an integrated luminosity of $36.1 \mathrm{fb}^{-1}$. Data are analysed in the lepton+jets final state, characterised by an isolated electron or muon with high transverse momentum, large missing transverse momentum and multiple jets, as well as the jets $+E_{\mathrm{T}}^{\text {miss }}$ final state, characterised by multiple jets and large missing transverse momentum. The search exploits the high multiplicity of $b$-jets, the high scalar sum of transverse momenta of all final-state objects, and the presence of boosted, hadronically decaying top quarks and Higgs bosons reconstructed as large-radius jets, characteristic of signal events.

No significant excess of events above the Standard Model expectation is observed, and 95\% CL lower limits are placed on the mass of the vector-like $T$ quark under several branching ratio hypotheses assuming contributions only from $T \rightarrow W b, Z t, H t$. The 95\% CL observed lower limits on the $T$ quark mass lie between $0.99 \mathrm{TeV}$ and $1.43 \mathrm{TeV}$ depending on the values of the branching ratios into the three decay modes. Assuming $\mathcal{B}(T \rightarrow H t)=1$ and $\mathcal{B}(T \rightarrow Z t)=1$, observed (expected) $95 \%$ CL limits of $m_{T}>1.43 \mathrm{TeV}(1.34 \mathrm{TeV}) \mathrm{TeV}$ and $m_{T}>1.17(1.18) \mathrm{TeV}$, respectively, are obtained. The observed (expected) 95\% CL limits for a weak-isospin doublet and singlet are $m_{T}>1.31(1.26) \mathrm{TeV}$ and $m_{T}>1.19(1.11) \mathrm{TeV}$, respectively. Additionally, upper limits on the four-top-quark production cross section are set in several new physics scenarios. In the case of $t \bar{t} t \bar{t}$ production from a contact interaction in an EFT model, the observed (expected) 95\% CL upper limit on the production cross section is $16 \mathrm{fb}\left(31_{-9}^{+12} \mathrm{fb}\right)$. In the context of a $2 \mathrm{UED} / \mathrm{RPP}$ model, $95 \% \mathrm{CL}$ observed (expected) lower limits on $m_{K K}$ of $1.8 \mathrm{TeV}(1.7 \mathrm{TeV})$ are derived.

\section{Acknowledgments}

We thank CERN for the very successful operation of the LHC, as well as the support staff from our institutions without whom ATLAS could not be operated efficiently.

We acknowledge the support of ANPCyT, Argentina; YerPhI, Armenia; ARC, Australia; BMWFW and FWF, Austria; ANAS, Azerbaijan; SSTC, Belarus; CNPq and FAPESP, Brazil; NSERC, NRC and CFI, Canada; CERN; CONICYT, Chile; CAS, MOST and NSFC, China; COLCIENCIAS, Colombia; MSMT CR, MPO CR and VSC CR, Czech Republic; DNRF and DNSRC, Denmark; IN2P3-CNRS, CEA-DRF/IRFU, France; SRNSFG, Georgia; BMBF, HGF, and MPG, Germany; GSRT, Greece; RGC, Hong Kong SAR, China; ISF, I-CORE and Benoziyo Center, Israel; INFN, Italy; MEXT and JSPS, Japan; CNRST, Morocco; NWO, Netherlands; RCN, Norway; MNiSW and NCN, Poland; FCT, Portugal; MNE/IFA, Romania; MES of Russia and NRC KI, Russian Federation; JINR; MESTD, Serbia; MSSR, Slovakia; ARRS and MIZŠ, Slovenia; DST/NRF, South Africa; MINECO, Spain; SRC and Wallenberg Foundation, Sweden; SERI, SNSF and 
Cantons of Bern and Geneva, Switzerland; MOST, Taiwan; TAEK, Turkey; STFC, United Kingdom; DOE and NSF, United States of America. In addition, individual groups and members have received support from BCKDF, the Canada Council, CANARIE, CRC, Compute Canada, FQRNT, and the Ontario Innovation Trust, Canada; EPLANET, ERC, ERDF, FP7, Horizon 2020 and Marie Skłodowska-Curie Actions, European Union; Investissements d'Avenir Labex and Idex, ANR, Région Auvergne and Fondation Partager le Savoir, France; DFG and AvH Foundation, Germany; Herakleitos, Thales and Aristeia programmes co-financed by EU-ESF and the Greek NSRF; BSF, GIF and Minerva, Israel; BRF, Norway; CERCA Programme Generalitat de Catalunya, Generalitat Valenciana, Spain; the Royal Society and Leverhulme Trust, United Kingdom.

The crucial computing support from all WLCG partners is acknowledged gratefully, in particular from CERN, the ATLAS Tier-1 facilities at TRIUMF (Canada), NDGF (Denmark, Norway, Sweden), CC-IN2P3 (France), KIT/GridKA (Germany), INFN-CNAF (Italy), NL-T1 (Netherlands), PIC (Spain), ASGC (Taiwan), RAL (U.K.) and BNL (U.S.A.), the Tier-2 facilities worldwide and large non-WLCG resource providers. Major contributors of computing resources are listed in ref. [117].

Open Access. This article is distributed under the terms of the Creative Commons Attribution License (CC-BY 4.0), which permits any use, distribution and reproduction in any medium, provided the original author(s) and source are credited.

\section{References}

[1] ATLAS collaboration, Observation of a new particle in the search for the Standard Model Higgs boson with the ATLAS detector at the LHC, Phys. Lett. B 716 (2012) 1 [arXiv: 1207.7214] [INSPIRE].

[2] CMS collaboration, Observation of a new boson at a mass of $125 \mathrm{GeV}$ with the CMS experiment at the LHC, Phys. Lett. B 716 (2012) 30 [arXiv:1207.7235] [INSPIRE].

[3] L. Susskind, Dynamics of spontaneous symmetry breaking in the Weinberg-Salam theory, Phys. Rev. D 20 (1979) 2619 [INSPIRE].

[4] C.T. Hill and E.H. Simmons, Strong dynamics and electroweak symmetry breaking, Phys. Rept. 381 (2003) 235 [Erratum ibid. 390 (2004) 553] [hep-ph/0203079] [INSPIRE].

[5] D.B. Kaplan, H. Georgi and S. Dimopoulos, Composite Higgs scalars, Phys. Lett. B 136 (1984) 187 [INSPIRE].

[6] K. Agashe, R. Contino and A. Pomarol, The minimal composite Higgs model, Nucl. Phys. B 719 (2005) 165 [hep-ph/0412089] [INSPIRE].

[7] F. del Aguila and M.J. Bowick, The possibility of new fermions with $\Delta I=0$ mass, Nucl. Phys. B 224 (1983) 107 [INSPIRE].

[8] J.A. Aguilar-Saavedra, Identifying top partners at LHC, JHEP 11 (2009) 030 [arXiv:0907.3155] [INSPIRE].

[9] J.A. Aguilar-Saavedra, Mixing with vector-like quarks: constraints and expectations, EPJ Web Conf. 60 (2013) 16012 [arXiv:1306.4432] [INSPIRE]. 
[10] J.A. Aguilar-Saavedra, R. Benbrik, S. Heinemeyer and M. Pérez-Victoria, Handbook of vectorlike quarks: mixing and single production, Phys. Rev. D 88 (2013) 094010 [arXiv:1306.0572] [INSPIRE].

[11] A. Pomarol and J. Serra, Top quark compositeness: feasibility and implications, Phys. Rev. D 78 (2008) 074026 [arXiv:0806.3247] [INSPIRE].

[12] B. Lillie, J. Shu and T.M.P. Tait, Top compositeness at the Tevatron and LHC, JHEP 04 (2008) 087 [arXiv:0712.3057] [INSPIRE].

[13] K. Kumar, T.M.P. Tait and R. Vega-Morales, Manifestations of top compositeness at colliders, JHEP 05 (2009) 022 [arXiv:0901.3808] [INSPIRE].

[14] G. Cacciapaglia, H. Cai, A. Deandrea, T. Flacke, S.J. Lee and A. Parolini, Composite scalars at the LHC: the Higgs, the sextet and the octet, JHEP 11 (2015) 201 [arXiv: 1507.02283] [INSPIRE].

[15] M. Guchait, F. Mahmoudi and K. Sridhar, Associated production of a Kaluza-Klein

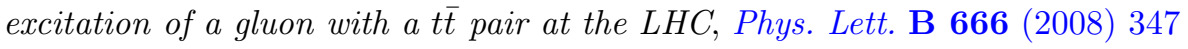
[arXiv:0710.2234] [INSPIRE].

[16] H. Georgi, L. Kaplan, D. Morin and A. Schenk, Effects of top compositeness, Phys. Rev. D 51 (1995) 3888 [hep-ph/9410307] [INSPIRE].

[17] C. Degrande, J.-M. Gerard, C. Grojean, F. Maltoni and G. Servant, Non-resonant new physics in top pair production at hadron colliders, JHEP 03 (2011) 125 [arXiv: 1010.6304] [INSPIRE].

[18] G. Cacciapaglia, A. Deandrea and J. Llodra-Perez, A dark matter candidate from Lorentz invariance in 6D, JHEP 03 (2010) 083 [arXiv:0907.4993] [INSPIRE].

[19] G. Cacciapaglia, R. Chierici, A. Deandrea, L. Panizzi, S. Perries and S. Tosi, Four tops on the real projective plane at LHC, JHEP 10 (2011) 042 [arXiv:1107.4616] [INSPIRE].

[20] ATLAS collaboration, Search for pair production of vector-like top quarks in events with one lepton, jets and missing transverse momentum in $\sqrt{s}=13$ TeV pp collisions with the ATLAS detector, JHEP 08 (2017) 052 [arXiv: 1705.10751] [INSPIRE].

[21] ATLAS collaboration, Search for pair production of heavy vector-like quarks decaying to high-p $p_{T} W$ bosons and $b$ quarks in the lepton-plus-jets final state in pp collisions at $\sqrt{s}=13 \mathrm{TeV}$ with the ATLAS detector, JHEP 10 (2017) 141 [arXiv:1707.03347] [INSPIRE].

[22] CMS collaboration, Search for pair production of vector-like $T$ and $B$ quarks in single-lepton final states using boosted jet substructure in proton-proton collisions at $\sqrt{s}=13$ TeV, JHEP 11 (2017) 085 [arXiv: 1706.03408] [INSPIRE].

[23] CMS collaboration, Search for pair production of vector-like quarks in the bW $\bar{b} W$ channel from proton-proton collisions at $\sqrt{s}=13$ TeV, Phys. Lett. B 779 (2018) 82 [arXiv: 1710.01539] [INSPIRE].

[24] ATLAS collaboration, Analysis of events with b-jets and a pair of leptons of the same charge in pp collisions at $\sqrt{s}=8$ TeV with the ATLAS detector, JHEP 10 (2015) 150 [arXiv: 1504.04605] [INSPIRE].

[25] ATLAS collaboration, Search for production of vector-like quark pairs and of four top quarks in the lepton-plus-jets final state in pp collisions at $\sqrt{s}=8 \mathrm{TeV}$ with the ATLAS detector, JHEP 08 (2015) 105 [arXiv: 1505.04306] [INSPIRE]. 
[26] CMS collaboration, Search for Standard Model production of four top quarks with same-sign and multilepton final states in proton-proton collisions at $\sqrt{s}=13 \mathrm{TeV}$, Eur. Phys. J. C 78 (2018) 140 [arXiv:1710.10614] [INSPIRE].

[27] ATLAS collaboration, The ATLAS experiment at the CERN Large Hadron Collider, 2008 JINST 3 S08003 [INSPIRE].

[28] ATLAS collaboration, ATLAS insertable B-layer technical design report, CERN-LHCC-2010-013, CERN, Geneva, Switzerland, (2010) [ATLAS-TDR-19].

[29] ATLAS collaboration, Performance of the ATLAS trigger system in 2015, Eur. Phys. J. C 77 (2017) 317 [arXiv: 1611.09661] [INSPIRE].

[30] ATLAS collaboration, Vertex reconstruction performance of the ATLAS detector at $\sqrt{s}=13 \mathrm{TeV}$, ATL-PHYS-PUB-2015-026, CERN, Geneva, Switzerland, (2015).

[31] ATLAS collaboration, Electron efficiency measurements with the ATLAS detector using the 2015 LHC proton-proton collision data, ATLAS-CONF-2016-024, CERN, Geneva, Switzerland, (2016).

[32] ATLAS collaboration, Electron and photon energy calibration with the ATLAS detector using data collected in 2015 at $\sqrt{s}=13 \mathrm{TeV}$, ATL-PHYS-PUB-2016-015, CERN, Geneva, Switzerland, (2016).

[33] ATLAS collaboration, Muon reconstruction performance of the ATLAS detector in proton-proton collision data at $\sqrt{s}=13$ TeV, Eur. Phys. J. C 76 (2016) 292 [arXiv: 1603.05598] [INSPIRE].

[34] M. Cacciari, G.P. Salam and G. Soyez, The anti-k $k_{t}$ jet clustering algorithm, JHEP 04 (2008) 063 [arXiv: 0802.1189] [InSPIRE].

[35] M. Cacciari and G.P. Salam, Dispelling the $N^{3}$ myth for the $k_{t}$ jet-finder, Phys. Lett. B 641 (2006) 57 [hep-ph/0512210] [INSPIRE].

[36] M. Cacciari, G.P. Salam and G. Soyez, FastJet user manual, Eur. Phys. J. C 72 (2012) 1896 [arXiv: 1111.6097] [INSPIRE].

[37] ATLAS collaboration, Topological cell clustering in the ATLAS calorimeters and its performance in LHC run 1, Eur. Phys. J. C 77 (2017) 490 [arXiv: 1603. 02934] [InSPIRE].

[38] ATLAS collaboration, Jet energy scale measurements and their systematic uncertainties in proton-proton collisions at $\sqrt{s}=13 \mathrm{TeV}$ with the ATLAS detector, Phys. Rev. D 96 (2017) 072002 [arXiv: 1703.09665] [INSPIRE].

[39] ATLAS collaboration, Selection of jets produced in $13 \mathrm{TeV}$ proton-proton collisions with the ATLAS detector, ATLAS-CONF-2015-029, CERN, Geneva, Switzerland, (2015).

[40] ATLAS collaboration, Performance of pile-up mitigation techniques for jets in pp collisions at $\sqrt{s}=8 \mathrm{TeV}$ using the ATLAS detector, Eur. Phys. J. C 76 (2016) 581 [arXiv: 1510.03823] [INSPIRE].

[41] ATLAS collaboration, Performance of b-jet identification in the ATLAS experiment, 2016 JINST 11 P04008 [arXiv:1512.01094] [INSPIRE].

[42] ATLAS collaboration, Optimisation of the ATLAS b-tagging performance for the 2016 LHC run, ATL-PHYS-PUB-2016-012, CERN, Geneva, Switzerland, (2016). 
[43] B. Nachman, P. Nef, A. Schwartzman, M. Swiatlowski and C. Wanotayaroj, Jets from jets: re-clustering as a tool for large radius jet reconstruction and grooming at the LHC, JHEP 02 (2015) 075 [arXiv: 1407.2922] [INSPIRE].

[44] D. Krohn, J. Thaler and L.-T. Wang, Jet trimming, JHEP 02 (2010) 084 [arXiv: 0912.1342] [INSPIRE].

[45] ATLAS collaboration, Performance of algorithms that reconstruct missing transverse momentum in $\sqrt{s}=8 \mathrm{TeV}$ proton-proton collisions in the ATLAS detector, Eur. Phys. J. C 77 (2017) 241 [arXiv: 1609.09324] [INSPIRE].

[46] ATLAS collaboration, Performance of missing transverse momentum reconstruction for the ATLAS detector in the first proton-proton collisions at at $\sqrt{s}=13 \mathrm{TeV}$, ATL-PHYS-PUB-2015-027, CERN, Geneva, Switzerland, (2015).

[47] T. Gleisberg et al., Event generation with SHERPA 1.1, JHEP 02 (2009) 007 [arXiv:0811.4622] [INSPIRE].

[48] D.J. Lange, The EvtGen particle decay simulation package, Nucl. Instrum. Meth. A 462 (2001) 152 [INSPIRE].

[49] T. Sjöstrand, S. Mrenna and P.Z. Skands, A brief introduction to PYTHIA 8.1, Comput. Phys. Commun. 178 (2008) 852 [arXiv:0710.3820] [InSPIRE].

[50] ATLAS collaboration, The ATLAS simulation infrastructure, Eur. Phys. J. C 70 (2010) 823 [arXiv: 1005.4568] [INSPIRE].

[51] GEANT4 collaboration, S. Agostinelli et al., GEANT4: a simulation toolkit, Nucl. Instrum. Meth. A 506 (2003) 250 [INSPIRE].

[52] ATLAS collaboration, The simulation principle and performance of the ATLAS fast calorimeter simulation FastCaloSim, ATL-PHYS-PUB-2010-013, CERN, Geneva, Switzerland, (2010).

[53] J.A. Aguilar-Saavedra, PROTOS, a PROgram for TOp Simulations, http://jaguilar.web.cern.ch/jaguilar/protos/.

[54] R.D. Ball et al., Parton distributions with LHC data, Nucl. Phys. B 867 (2013) 244 [arXiv:1207.1303] [INSPIRE].

[55] ATLAS collaboration, ATLAS run 1 PYTHIA8 tunes, ATL-PHYS-PUB-2014-021, CERN, Geneva, Switzerland, (2014).

[56] M. Czakon and A. Mitov, Top++: a program for the calculation of the top-pair cross-section at hadron colliders, Comput. Phys. Commun. 185 (2014) 2930 [arXiv:1112.5675] [INSPIRE].

[57] M. Cacciari, M. Czakon, M. Mangano, A. Mitov and P. Nason, Top-pair production at hadron colliders with next-to-next-to-leading logarithmic soft-gluon resummation, Phys. Lett. B 710 (2012) 612 [arXiv:1111.5869] [INSPIRE].

[58] P. Bärnreuther, M. Czakon and A. Mitov, Percent level precision physics at the Tevatron: first genuine NNLO QCD corrections to $q \bar{q} \rightarrow t \bar{t}+X$, Phys. Rev. Lett. 109 (2012) 132001 [arXiv: 1204.5201] [INSPIRE].

[59] M. Czakon and A. Mitov, NNLO corrections to top-pair production at hadron colliders: the all-fermionic scattering channels, JHEP 12 (2012) 054 [arXiv:1207.0236] [INSPIRE]. 
[60] M. Czakon and A. Mitov, NNLO corrections to top pair production at hadron colliders: the quark-gluon reaction, JHEP 01 (2013) 080 [arXiv: 1210.6832] [INSPIRE].

[61] M. Czakon, P. Fiedler and A. Mitov, Total top-quark pair-production cross section at hadron colliders through $O\left(\alpha_{S}^{4}\right)$, Phys. Rev. Lett. 110 (2013) 252004 [arXiv:1303.6254] [INSPIRE].

[62] A.D. Martin, W.J. Stirling, R.S. Thorne and G. Watt, Parton distributions for the LHC, Eur. Phys. J. C 63 (2009) 189 [arXiv:0901.0002] [INSPIRE].

[63] A.D. Martin, W.J. Stirling, R.S. Thorne and G. Watt, Uncertainties on $\alpha_{S}$ in global PDF analyses and implications for predicted hadronic cross sections, Eur. Phys. J. C 64 (2009) 653 [arXiv: 0905.3531] [INSPIRE].

[64] M. Botje et al., The PDF4LHC working group interim recommendations, arXiv:1101.0538 [INSPIRE].

[65] H.-L. Lai et al., New parton distributions for collider physics, Phys. Rev. D 82 (2010) 074024 [arXiv: 1007.2241 ] [INSPIRE].

[66] J. Gao et al., CT10 next-to-next-to-leading order global analysis of QCD, Phys. Rev. D 89 (2014) 033009 [arXiv:1302.6246] [INSPIRE].

[67] J. Alwall et al., The automated computation of tree-level and next-to-leading order differential cross sections and their matching to parton shower simulations, JHEP $\mathbf{0 7}$ (2014) 079 [arXiv: 1405.0301] [InSPIRE].

[68] P. Meade and M. Reece, BRIDGE: Branching Ratio Inquiry/Decay Generated Events, hep-ph/0703031 [INSPIRE].

[69] S. Frixione, P. Nason and G. Ridolfi, A positive-weight next-to-leading-order Monte Carlo for heavy flavour hadroproduction, JHEP 09 (2007) 126 [arXiv:0707.3088] [INSPIRE].

[70] P. Nason, A new method for combining NLO QCD with shower Monte Carlo algorithms, JHEP 11 (2004) 040 [hep-ph/0409146] [INSPIRE].

[71] S. Frixione, P. Nason and C. Oleari, Matching NLO QCD computations with parton shower simulations: the POWHEG method, JHEP 11 (2007) 070 [arXiv: 0709. 2092] [INSPIRE].

[72] S. Alioli, P. Nason, C. Oleari and E. Re, A general framework for implementing NLO calculations in shower Monte Carlo programs: the POWHEG BOX, JHEP 06 (2010) 043 [arXiv: 1002 .2581] [INSPIRE].

[73] ATLAS collaboration, Comparison of Monte Carlo generator predictions to ATLAS measurements of top pair production at $7 \mathrm{TeV}$, ATL-PHYS-PUB-2015-002, CERN, Geneva, Switzerland, (2015).

[74] T. Sjöstrand, S. Mrenna and P.Z. Skands, PYTHIA 6.4 physics and manual, JHEP 05 (2006) 026 [hep-ph/0603175] [INSPIRE].

[75] P.Z. Skands, Tuning Monte Carlo generators: the Perugia tunes, Phys. Rev. D 82 (2010) 074018 [arXiv: 1005.3457] [INSPIRE].

[76] ATLAS collaboration, Search for the Standard Model Higgs boson produced in association with top quarks and decaying into b $\bar{b}$ in pp collisions at $\sqrt{s}=8 \mathrm{TeV}$ with the ATLAS detector, Eur. Phys. J. C 75 (2015) 349 [arXiv:1503.05066] [INSPIRE].

[77] ATLAS collaboration, Measurements of top-quark pair differential cross-sections in the lepton+jets channel in pp collisions at $\sqrt{s}=8 \mathrm{TeV}$ using the ATLAS detector, Eur. Phys. J. C 76 (2016) 538 [arXiv:1511.04716] [InSPIRE]. 
[78] M. Czakon, D. Heymes and A. Mitov, High-precision differential predictions for top-quark pairs at the LHC, Phys. Rev. Lett. 116 (2016) 082003 [arXiv:1511.00549] [InSPIRE].

[79] M. Czakon, D. Heymes and A. Mitov, Dynamical scales for multi-TeV top-pair production at the LHC, JHEP 04 (2017) 071 [arXiv: 1606. 03350] [INSPIRE].

[80] F. Cascioli, P. Maierhöfer, N. Moretti, S. Pozzorini and F. Siegert, NLO matching for $t \bar{t} b \bar{b}$ production with massive b-quarks, Phys. Lett. B 734 (2014) 210 [arXiv:1309.5912] [INSPIRE].

[81] F. Cascioli, P. Maierhofer and S. Pozzorini, Scattering amplitudes with open loops, Phys. Rev. Lett. 108 (2012) 111601 [arXiv:1111.5206] [INSPIRE].

[82] R. Frederix, E. Re and P. Torrielli, Single-top t-channel hadroproduction in the four-flavour scheme with POWHEG and aMC@NLO, JHEP 09 (2012) 130 [arXiv:1207.5391] [INSPIRE].

[83] S. Frixione, E. Laenen, P. Motylinski and B.R. Webber, Single-top production in MC@NLO, JHEP 03 (2006) 092 [hep-ph/0512250] [INSPIRE].

[84] N. Kidonakis, Next-to-next-to-leading-order collinear and soft gluon corrections for t-channel single top quark production, Phys. Rev. D 83 (2011) 091503 [arXiv:1103.2792] [INSPIRE].

[85] N. Kidonakis, Two-loop soft anomalous dimensions for single top quark associated production with a $W^{-}$or $H^{-}$, Phys. Rev. D 82 (2010) 054018 [arXiv: 1005.4451] [INSPIRE].

[86] N. Kidonakis, NNLL resummation for s-channel single top quark production, Phys. Rev. D 81 (2010) 054028 [arXiv: 1001.5034] [INSPIRE].

[87] T. Gleisberg and S. Höche, Comix, a new matrix element generator, JHEP 12 (2008) 039 [arXiv:0808.3674] [INSPIRE].

[88] S. Schumann and F. Krauss, A parton shower algorithm based on Catani-Seymour dipole factorisation, JHEP 03 (2008) 038 [arXiv:0709.1027] [inSPIRE].

[89] S. Höche, F. Krauss, M. Schönherr and F. Siegert, QCD matrix elements + parton showers: the NLO case, JHEP 04 (2013) 027 [arXiv:1207.5030] [INSPIRE].

[90] NNPDF collaboration, R.D. Ball et al., Parton distributions for the LHC run II, JHEP 04 (2015) 040 [arXiv: 1410.8849] [INSPIRE].

[91] C. Anastasiou, L.J. Dixon, K. Melnikov and F. Petriello, High precision QCD at hadron colliders: electroweak gauge boson rapidity distributions at NNLO, Phys. Rev. D 69 (2004) 094008 [hep-ph/0312266] [INSPIRE].

[92] R. Raitio and W.W. Wada, Higgs boson production at large transverse momentum in QCD, Phys. Rev. D 19 (1979) 941 [INSPIRE].

[93] W. Beenakker, S. Dittmaier, M. Krämer, B. Plumper, M. Spira and P.M. Zerwas, NLO

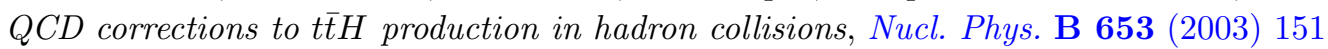
[hep-ph/0211352] [INSPIRE].

[94] S. Dawson, C. Jackson, L.H. Orr, L. Reina and D. Wackeroth, Associated Higgs production with top quarks at the Large Hadron Collider: NLO QCD corrections, Phys. Rev. D 68 (2003) 034022 [hep-ph/0305087] [INSPIRE]. 
[95] Y. Zhang, W.-G. Ma, R.-Y. Zhang, C. Chen and L. Guo, $Q C D N L O$ and EW NLO corrections to $t \bar{t} H$ production with top quark decays at hadron collider, Phys. Lett. B 738 (2014) 1 [arXiv:1407.1110] [INSPIRE].

[96] S. Frixione, V. Hirschi, D. Pagani, H.-S. Shao and M. Zaro, Electroweak and QCD corrections to top-pair hadroproduction in association with heavy bosons, JHEP 06 (2015) 184 [arXiv: 1504.03446] [INSPIRE].

[97] A. Djouadi, J. Kalinowski and M. Spira, HDECAY: a program for Higgs boson decays in the Standard Model and its supersymmetric extension, Comput. Phys. Commun. 108 (1998) 56 [hep-ph/9704448] [INSPIRE].

[98] ATLAS collaboration, Measurement of the top quark-pair production cross section with ATLAS in pp collisions at $\sqrt{s}=7$ TeV, Eur. Phys. J. C 71 (2011) 1577 [arXiv:1012.1792] [INSPIRE].

[99] ATLAS collaboration, Luminosity determination in pp collisions at $\sqrt{s}=8 \mathrm{TeV}$ using the ATLAS detector at the LHC, Eur. Phys. J. C 76 (2016) 653 [arXiv:1608.03953] [inSPIRE].

[100] M. Bahr et al., HERWIG++ physics and manual, Eur. Phys. J. C 58 (2008) 639 [arXiv:0803.0883] [INSPIRE].

[101] ATLAS collaboration, Simulation of top quark production for the ATLAS experiment at $\sqrt{s}=13 \mathrm{TeV}$, ATL-PHYS-PUB-2016-004, CERN, Geneva, Switzerland, (2016).

[102] ATLAS collaboration, Search for the Standard Model Higgs boson produced in association with top quarks and decaying into a b $\bar{b}$ pair in pp collisions at $\sqrt{s}=13 \mathrm{TeV}$ with the ATLAS detector, Phys. Rev. D 97 (2018) 072016 [arXiv:1712.08895] [INSPIRE].

[103] LHC Higgs Cross Section Working Group collaboration, D. de Florian et al., Handbook of LHC Higgs cross sections: 4. Deciphering the nature of the Higgs sector, arXiv: 1610.07922 [INSPIRE].

[104] S. Frixione, E. Laenen, P. Motylinski, B.R. Webber and C.D. White, Single-top hadroproduction in association with a W boson, JHEP 07 (2008) 029 [arXiv:0805.3067] [INSPIRE].

[105] ATLAS collaboration, Evidence for the $H \rightarrow b \bar{b}$ decay with the ATLAS detector, JHEP 12 (2017) 024 [arXiv: 1708.03299] [INSPIRE].

[106] J.M. Campbell and R.K. Ellis, An update on vector boson pair production at hadron colliders, Phys. Rev. D 60 (1999) 113006 [hep-ph/9905386] [INSPIRE].

[107] J. Alwall et al., Comparative study of various algorithms for the merging of parton showers and matrix elements in hadronic collisions, Eur. Phys. J. C 53 (2008) 473 [arXiv:0706.2569] [INSPIRE].

[108] ATLAS collaboration, Measurement of the $W^{ \pm} Z$ boson pair-production cross section in pp collisions at $\sqrt{s}=13 \mathrm{TeV}$ with the ATLAS Detector, Phys. Lett. B 762 (2016) 1 [arXiv: 1606.04017] [INSPIRE].

[109] J.M. Campbell and R.K. Ellis, $t \bar{t} W^{ \pm}$production and decay at NLO, JHEP 07 (2012) 052 [arXiv: 1204.5678] [INSPIRE].

[110] M.V. Garzelli, A. Kardos, C.G. Papadopoulos and Z. Trócsányi, $t \bar{t} W^{ \pm}$and $t \bar{t} Z$ hadroproduction at NLO accuracy in QCD with parton shower and hadronization effects, JHEP 11 (2012) 056 [arXiv:1208.2665] [INSPIRE]. 
[111] LHC Higgs Cross Section Working Group collaboration, S. Dittmaier et al., Handbook of LHC Higgs cross sections: 1. Inclusive observables, arXiv:1101.0593 [INSPIRE].

[112] W. Verkerke and D.P. Kirkby, The RooFit toolkit for data modeling, eConf C 0303241 (2003) MOLT007 [physics/0306116] [INSPIRE].

[113] W. Verkerke and D. Kirkby, RooFit users manual, http://roofit.sourceforge.net/.

[114] G. Cowan, K. Cranmer, E. Gross and O. Vitells, Asymptotic formulae for likelihood-based tests of new physics, Eur. Phys. J. C 71 (2011) 1554 [Erratum ibid. C 73 (2013) 2501] [arXiv: 1007.1727] [INSPIRE].

[115] T. Junk, Confidence level computation for combining searches with small statistics, Nucl. Instrum. Meth. A 434 (1999) 435 [hep-ex/9902006] [INSPIRE].

[116] A.L. Read, Presentation of search results: the $C L_{S}$ technique, J. Phys. G 28 (2002) 2693 [INSPIRE].

[117] ATLAS collaboration, ATLAS computing acknowledgements, ATL-GEN-PUB-2016-002, CERN, Geneva, Switzerland, (2016). 


\section{The ATLAS collaboration}

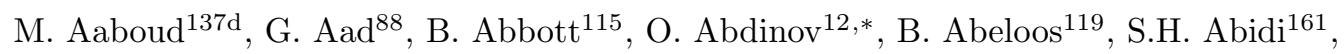
O.S. AbouZeid ${ }^{139}$, N.L. Abraham ${ }^{151}$, H. Abramowicz ${ }^{155}$, H. Abreu ${ }^{154}$, Y. Abulaiti ${ }^{6}$, B.S. Acharya ${ }^{167 a, 167 b, a}$, S. Adachi ${ }^{157}$, L. Adamczyk ${ }^{41 a}$, J. Adelman ${ }^{110}$, M. Adersberger ${ }^{102}$, T. Adye ${ }^{133}$, A.A. Affolder ${ }^{139}$, Y. Afik ${ }^{154}$, C. Agheorghiesei ${ }^{28 c}$, J.A. Aguilar-Saavedra ${ }^{128 a, 128 f}$, F. Ahmadov ${ }^{68, b}$, G. Aielli135a,135b, S. Akatsuka ${ }^{71}$, T.P.A. Åkesson ${ }^{84}$, E. Akilli ${ }^{52}$, A.V. Akimov ${ }^{98}$, G.L. Alberghi ${ }^{22 a, 22 b}$, J. Albert ${ }^{172}$, P. Albicocco ${ }^{50}$, M.J. Alconada Verzini ${ }^{74}$, S. Alderweireldt ${ }^{108}$, M. Aleksa ${ }^{32}$, I.N. Aleksandrov ${ }^{68}$, C. Alexa ${ }^{28 b}$, G. Alexander ${ }^{155}$, T. Alexopoulos ${ }^{10}$, M. Alhroob $^{115}$, B. Ali ${ }^{130}$, M. Aliev $76 a, 76 b$, G. Alimonti ${ }^{94 a}$, J. Alison ${ }^{33}$, S.P. Alkire ${ }^{140}$, C. Allaire ${ }^{119}$,

B.M.M. Allbrooke ${ }^{151}$, B.W. Allen ${ }^{118}$, P.P. Allport ${ }^{19}$, A. Aloisio 106a,106b, A. Alonso ${ }^{39}$, F. Alonso ${ }^{74}$, C. Alpigiani ${ }^{140}$, A.A. Alshehri ${ }^{56}$, M.I. Alstaty ${ }^{88}$, B. Alvarez Gonzalez ${ }^{32}$, D. Álvarez Piqueras ${ }^{170}$, M.G. Alviggi ${ }^{106 a, 106 b}$, B.T. Amadio ${ }^{16}$, Y. Amaral Coutinho ${ }^{26 a}$, L. Ambroz ${ }^{122}$, C. Amelung ${ }^{25}$, D. Amidei ${ }^{92}$, S.P. Amor Dos Santos ${ }^{128 a, 128 c}$, S. Amoroso ${ }^{32}$, C.S. Amrouche ${ }^{52}$, C. Anastopoulos ${ }^{141}$, L.S. Ancu ${ }^{52}$, N. Andari ${ }^{19}$, T. Andeen ${ }^{11}$, C.F. Anders ${ }^{60 b}$, J.K. Anders ${ }^{18}$, K.J. Anderson ${ }^{33}$, A. Andreazza ${ }^{94 a, 94 b}$, V. Andrei ${ }^{60 a}$, S. Angelidakis ${ }^{37}$, I. Angelozzi ${ }^{109}$, A. Angerami ${ }^{38}$, A.V. Anisenkov ${ }^{111, c}$, A. Annovi ${ }^{126 a}$, C. Antel ${ }^{60 a}$, M.T. Anthony ${ }^{141}$, M. Antonelli $^{50}$, D.J.A. Antrim ${ }^{166}$, F. Anulli ${ }^{134 a}$, M. Aoki ${ }^{69}$, L. Aperio Bella ${ }^{32}$, G. Arabidze ${ }^{93}$, Y. Arai ${ }^{69}$, J.P. Araque ${ }^{128 a}$, V. Araujo Ferraz ${ }^{26 a}$, R. Araujo Pereira ${ }^{26 a}$, A.T.H. Arce ${ }^{48}$, R.E. Ardell ${ }^{80}$, F.A. Arduh ${ }^{74}$, J-F. Arguin ${ }^{97}$, S. Argyropoulos ${ }^{66}$, A.J. Armbruster ${ }^{32}$, L.J. Armitage ${ }^{79}$,

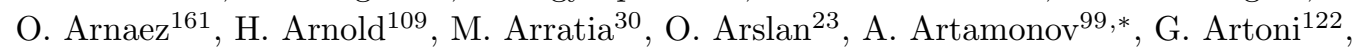
S. Artz ${ }^{86}$, S. Asai ${ }^{157}$, N. Asbah ${ }^{45}$, A. Ashkenazi ${ }^{155}$, E.M. Asimakopoulou ${ }^{168}$, L. Asquith ${ }^{151}$, K. Assamagan 27 , R. Astalos ${ }^{146 a}$, R.J. Atkin ${ }^{147 a}$, M. Atkinson ${ }^{169}$, N.B. Atlay ${ }^{143}$, K. Augsten ${ }^{130}$, G. Avolio ${ }^{32}$, R. Avramidou ${ }^{36 a}$, B. Axen ${ }^{16}$, M.K. Ayoub ${ }^{35 a}$, G. Azuelos ${ }^{97, d}$, A.E. Baas ${ }^{60 a}$, M.J. Baca ${ }^{19}$, H. Bachacou ${ }^{138}$, K. Bachas ${ }^{76 a, 76 b}$, M. Backes ${ }^{122}$, P. Bagnaia134a,134b, M. Bahmani ${ }^{42}$, H. Bahrasemani ${ }^{144}$, A.J. Bailey ${ }^{170}$, J.T. Baines ${ }^{133}$, M. Bajic ${ }^{39}$, O.K. Baker ${ }^{179}$, P.J. Bakker ${ }^{109}$, D. Bakshi Gupta ${ }^{82}$, E.M. Baldin ${ }^{111, c}$, P. Balek ${ }^{175}$, F. Balli ${ }^{138}$, W.K. Balunas ${ }^{124}$, E. Banas ${ }^{42}$, A. Bandyopadhyay ${ }^{23}$, Sw. Banerjee ${ }^{176, e}$, A.A.E. Bannoura ${ }^{177}$, L. Barak ${ }^{155}$, W.M. Barbe ${ }^{37}$, E.L. Barberio ${ }^{91}$, D. Barberis ${ }^{53 a, 53 b}$, M. Barbero ${ }^{88}$, T. Barillari ${ }^{103}$, M-S Barisits ${ }^{32}$,

J.T. Barkeloo ${ }^{118}$, T. Barklow ${ }^{145}$, N. Barlow ${ }^{30}$, R. Barnea ${ }^{154}$, S.L. Barnes ${ }^{36 c}$, B.M. Barnett ${ }^{133}$, R.M. Barnett ${ }^{16}$, Z. Barnovska-Blenessy ${ }^{36 a}$, A. Baroncelli ${ }^{136 a}$, G. Barone ${ }^{25}$, A.J. Barr ${ }^{122}$, L. Barranco Navarro ${ }^{170}$, F. Barreiro ${ }^{85}$, J. Barreiro Guimarães da Costa ${ }^{35 a}$, R. Bartoldus ${ }^{145}$, A.E. Barton ${ }^{75}$, P. Bartos ${ }^{146 a}$, A. Basalaev ${ }^{125}$, A. Bassalat ${ }^{119}$, R.L. Bates ${ }^{56}$, S.J. Batista ${ }^{161}$, S. Batlamous ${ }^{137 e}$, J.R. Batley ${ }^{30}$, M. Battaglia ${ }^{139}$, M. Bauce ${ }^{134 a, 134 b}$, F. Bauer ${ }^{138}$, K.T. Bauer ${ }^{166}$, H.S. Bawa ${ }^{145, f}$, J.B. Beacham ${ }^{113}$, M.D. Beattie ${ }^{75}$, T. Beau ${ }^{83}$, P.H. Beauchemin ${ }^{165}$, P. Bechtle ${ }^{23}$, H.P. Beck ${ }^{18, g}$, H.C. Beck ${ }^{58}$, K. Becker ${ }^{51}$, M. Becker ${ }^{86}$, C. Becot ${ }^{112}$, A.J. Beddall ${ }^{20 e}$, A. Beddall ${ }^{20 b}$, V.A. Bednyakov ${ }^{68}$, M. Bedognetti ${ }^{109}$, C.P. Bee ${ }^{150}$, T.A. Beermann ${ }^{32}$, M. Begalli ${ }^{26 a}$, M. Begel ${ }^{27}$, A. Behera ${ }^{150}$, J.K. Behr ${ }^{45}$, A.S. Bell ${ }^{81}$, G. Bella ${ }^{155}$, L. Bellagamba ${ }^{22 a}$, A. Bellerive ${ }^{31}$, M. Bellomo ${ }^{154}$, K. Belotskiy ${ }^{100}$, N.L. Belyaev ${ }^{100}$, O. Benary ${ }^{155, *}$,

D. Benchekroun ${ }^{137 a}$, M. Bender ${ }^{102}$, N. Benekos ${ }^{10}$, Y. Benhammou ${ }^{155}$, E. Benhar Noccioli ${ }^{179}$, J. Benitez ${ }^{66}$, D.P. Benjamin ${ }^{48}$, M. Benoit ${ }^{52}$, J.R. Bensinger ${ }^{25}$, S. Bentvelsen ${ }^{109}$, L. Beresford ${ }^{122}$, M. Beretta ${ }^{50}$, D. Berge ${ }^{45}$, E. Bergeaas Kuutmann ${ }^{168}$, N. Berger ${ }^{5}$, L.J. Bergsten ${ }^{25}$, J. Beringer ${ }^{16}$, S. Berlendis ${ }^{57}$, N.R. Bernard ${ }^{89}$, G. Bernardi ${ }^{83}$, C. Bernius ${ }^{145}$, F.U. Bernlochner ${ }^{23}$, T. Berry ${ }^{80}$, P. Berta ${ }^{86}$, C. Bertella ${ }^{35 a}$, G. Bertoli ${ }^{148 a, 148 b}$, I.A. Bertram ${ }^{75}$, C. Bertsche ${ }^{45}$, G.J. Besjes ${ }^{39}$, O. Bessidskaia Bylund ${ }^{148 a, 148 b}$, M. Bessner ${ }^{45}$, N. Besson ${ }^{138}$, A. Bethani ${ }^{87}$, S. Bethke $^{103}$,

A. Betti ${ }^{23}$, A.J. Bevan ${ }^{79}$, J. Beyer ${ }^{103}$, R.M. Bianchi ${ }^{127}$, O. Biebel ${ }^{102}$, D. Biedermann ${ }^{17}$,

R. Bielski ${ }^{87}$, K. Bierwagen ${ }^{86}$, N.V. Biesuz ${ }^{126 a, 126 b}$, M. Biglietti ${ }^{136 a}$, T.R.V. Billoud ${ }^{97}$, M. Bindi ${ }^{58}$, A. Bingul ${ }^{20 b}$, C. Bini ${ }^{134 a, 134 b}$, S. Biondi ${ }^{22 a, 22 b}$, T. Bisanz ${ }^{58}$, C. Bittrich $^{47}$, D.M. Bjergaard ${ }^{48}$, 
J.E. Black ${ }^{145}$, K.M. Black ${ }^{24}$, R.E. Blair ${ }^{6}$, T. Blazek ${ }^{146 a}$, I. Bloch ${ }^{45}$, C. Blocker $^{25}$, A. Blue ${ }^{56}$, U. Blumenschein ${ }^{79}$, Dr. Blunier ${ }^{34 a}$, G.J. Bobbink ${ }^{109}$, V.S. Bobrovnikov ${ }^{111, c}$, S.S. Bocchetta ${ }^{84}$, A. Bocci ${ }^{48}$, C. Bock ${ }^{102}$, D. Boerner ${ }^{177}$, D. Bogavac ${ }^{102}$, A.G. Bogdanchikov ${ }^{111}$, C. Bohm ${ }^{148 a}$, V. Boisvert ${ }^{80}$, P. Bokan ${ }^{168, h}$, T. Bold ${ }^{41 a}$, A.S. Boldyrev ${ }^{101}$, A.E. Bolz ${ }^{60 b}$, M. Bomben ${ }^{83}$, M. Bona ${ }^{79}$, J.S. Bonilla ${ }^{118}$, M. Boonekamp ${ }^{138}$, A. Borisov ${ }^{132}$, G. Borissov ${ }^{75}$, J. Bortfeldt ${ }^{32}$, D. Bortoletto ${ }^{122}$, V. Bortolotto ${ }^{135 a, 135 b}$, D. Boscherini ${ }^{22 a}$, M. Bosman ${ }^{13}$, J.D. Bossio Sola ${ }^{29}$, J. Boudreau ${ }^{127}$, E.V. Bouhova-Thacker ${ }^{75}$, D. Boumediene ${ }^{37}$, C. Bourdarios ${ }^{119}$, S.K. Boutle ${ }^{56}$, A. Boveia ${ }^{113}$, J. Boyd ${ }^{32}$, I.R. Boyko ${ }^{68}$, A.J. Bozson ${ }^{80}$, J. Bracinik ${ }^{19}$, N. Brahimi ${ }^{88}$, A. Brandt ${ }^{8}$, G. Brandt ${ }^{177}$, O. Brandt ${ }^{60 a}$, F. Braren ${ }^{45}$, U. Bratzler ${ }^{158}$, B. Brau ${ }^{89}$, J.E. Brau ${ }^{118}$, W.D. Breaden Madden ${ }^{56}$, K. Brendlinger ${ }^{45}$, A.J. Brennan ${ }^{91}$, L. Brenner ${ }^{45}$, R. Brenner ${ }^{168}$, S. Bressler ${ }^{175}$, B. Brickwedde ${ }^{86}$, D.L. Briglin ${ }^{19}$, T.M. Bristow ${ }^{49}$, D. Britton ${ }^{56}$, D. Britzger ${ }^{60 b}$, I. Brock $^{23}$, R. Brock ${ }^{93}$, G. Brooijmans ${ }^{38}$, T. Brooks ${ }^{80}$, W.K. Brooks ${ }^{34 b}$, E. Brost ${ }^{110}$, J.H Broughton ${ }^{19}$, P.A. Bruckman de Renstrom ${ }^{42}$, D. Bruncko ${ }^{146 b}$, A. Bruni ${ }^{22 a}$, G. Bruni2 ${ }^{22 a}$, L.S. Bruni ${ }^{109}$, S. Bruno ${ }^{135 a, 135 b}$, B.H. Brunt ${ }^{30}$, M. Bruschi ${ }^{22 a}$, N. Bruscino ${ }^{127}$, P. Bryant ${ }^{33}$, L. Bryngemark ${ }^{45}$, T. Buanes ${ }^{15}$, Q. Buat ${ }^{32}$, P. Buchholz ${ }^{143}$, A.G. Buckley ${ }^{56}$, I.A. Budagov ${ }^{68}$, F. Buehrer ${ }^{51}$, M.K. Bugge ${ }^{121}$, O. Bulekov ${ }^{100}$, D. Bullock ${ }^{8}$, T.J. Burch ${ }^{110}$, S. Burdin ${ }^{77}$, C.D. Burgard ${ }^{109}$, A.M. Burger ${ }^{5}$, B. Burghgrave ${ }^{110}$, K. Burka ${ }^{42}$, S. Burke ${ }^{133}$, I. Burmeister ${ }^{46}$, J.T.P. Burr ${ }^{122}$, D. Büscher ${ }^{51}$, V. Büscher ${ }^{86}$, E. Buschmann ${ }^{58}$, P. Bussey ${ }^{56}$, J.M. Butler ${ }^{24}$, C.M. Buttar ${ }^{56}$, J.M. Butterworth ${ }^{81}$, P. Butti ${ }^{32}$, W. Buttinger ${ }^{32}$, A. Buzatu ${ }^{153}$, A.R. Buzykaev ${ }^{111, c}$, G. Cabras ${ }^{22 a, 22 b}$, S. Cabrera Urbán ${ }^{170}$, D. Caforio ${ }^{130}$, H. Cai ${ }^{169}$, V.M.M. Cairo ${ }^{2}$, O. Cakir ${ }^{4 a}$, N. Calace ${ }^{52}$, P. Calafiura ${ }^{16}$, A. Calandri ${ }^{88}$, G. Calderini ${ }^{83}$, P. Calfayan ${ }^{64}$, G. Callea ${ }^{40 a, 40 b}$, L.P. Caloba ${ }^{26 a}$, S. Calvente Lopez $^{85}$, D. Calvet $^{37}$, S. Calvet ${ }^{37}$, T.P. Calvet ${ }^{150}$, M. Calvetti ${ }^{126 a, 126 b}$, R. Camacho Toro $^{33}$, S. Camarda ${ }^{32}$, P. Camarri ${ }^{135 a, 135 b}$, D. Cameron ${ }^{121}$, R. Caminal Armadans ${ }^{89}$, C. Camincher ${ }^{57}$, S. Campana ${ }^{32}$, M. Campanelli ${ }^{81}$, A. Camplani ${ }^{94 a, 94 b}$, A. Campoverde ${ }^{143}$, V. Canale ${ }^{106 a, 106 b}$, M. Cano Bret ${ }^{36 c}$, J. Cantero ${ }^{116}$, T. $\mathrm{Cao}^{155}$, Y. $\mathrm{Cao}^{169}$, M.D.M. Capeans Garrido ${ }^{32}$, I. Caprini' ${ }^{28 b}$, M. Caprini ${ }^{28 b}$, M. Capua ${ }^{40 a, 40 b}$ R.M. Carbone ${ }^{38}$, R. Cardarelli ${ }^{135 a}$, F. Cardillo ${ }^{51}$, I. Carli ${ }^{131}$, T. Carli ${ }^{32}$, G. Carlino ${ }^{106 a}$, B.T. Carlson ${ }^{127}$, L. Carminati ${ }^{94 a, 94 b}$, R.M.D. Carney ${ }^{148 a, 148 b}$, S. Caron ${ }^{108}$, E. Carquin ${ }^{34 b}$, S. Carrá ${ }^{94 a}, 94 b$, G.D. Carrillo-Montoya ${ }^{32}$, D. Casadei ${ }^{147 b}$, M.P. Casado ${ }^{13, i}$, A.F. Casha ${ }^{161}$, M. Casolino ${ }^{13}$, D.W. Casper ${ }^{166}$, R. Castelijn ${ }^{109}$, V. Castillo Gimenez ${ }^{170}$, N.F. Castro ${ }^{128 a, 128 e}$,

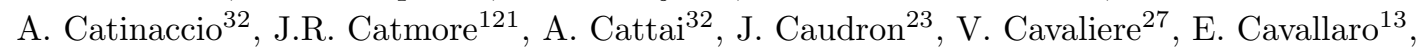
D. Cavalli ${ }^{94 a}$, M. Cavalli-Sforza ${ }^{13}$, V. Cavasinni ${ }^{126 a, 126 b}$, E. Celebi ${ }^{20 d}$, F. Ceradini ${ }^{136 a, 136 b}$, L. Cerda Alberich ${ }^{170}$, A.S. Cerqueira ${ }^{26 b}$, A. Cerri ${ }^{151}$, L. Cerrito ${ }^{135 a, 135 b}$, F. Cerutti ${ }^{16}$, A. Cervelli ${ }^{22 a}, 22 b$, S.A. Cetin ${ }^{20 d}$, A. Chafaq ${ }^{137 a}$, DC Chakraborty ${ }^{110}$, S.K. Chan ${ }^{59}$, W.S. Chan ${ }^{109}$, Y.L. Chan ${ }^{62 a}$, P. Chang ${ }^{169}$, J.D. Chapman ${ }^{30}$, D.G. Charlton ${ }^{19}$, C.C. $\mathrm{Chau}^{31}$,

C.A. Chavez Barajas ${ }^{151}$, S. Che ${ }^{113}$, A. Chegwidden ${ }^{93}$, S. Chekanov ${ }^{6}$, S.V. Chekulaev ${ }^{163 a}$, G.A. Chelkov ${ }^{68, j}$, M.A. Chelstowska ${ }^{32}$, C. Chen ${ }^{36 a}$, C. Chen ${ }^{67}$, H. Chen ${ }^{27}$, J. Chen ${ }^{36 a}$, J. Chen ${ }^{38}$, S. Chen ${ }^{35 b}$, S. Chen ${ }^{124}$, X. Chen ${ }^{35 c, k}$, Y. Chen ${ }^{70}$, Y.-H. Chen ${ }^{45}$, H.C. Cheng ${ }^{92}$, H.J. Cheng ${ }^{35 a, 35 d}$, A. Cheplakov ${ }^{68}$, E. Cheremushkina ${ }^{132}$, R. Cherkaoui El Moursli ${ }^{137 e}$, E. Cheu ${ }^{7}$, K. Cheung ${ }^{63}$, L. Chevalier ${ }^{138}$, V. Chiarella ${ }^{50}$, G. Chiarelli ${ }^{126 a}$, G. Chiodini ${ }^{76 a}$, A.S. Chisholm ${ }^{32}$, A. Chitan ${ }^{28 b}$, I. Chiu ${ }^{157}$, Y.H. Chiu ${ }^{172}$, M.V. Chizhov ${ }^{68}$, K. Choi ${ }^{64}$, A.R. Chomont ${ }^{119}$, S. Chouridou ${ }^{156}$, Y.S. Chow $^{109}$, V. Christodoulou ${ }^{81}$, M.C. Chu ${ }^{62 a}$, J. Chudoba ${ }^{129}$, A.J. Chuinard ${ }^{90}$, J.J. Chwastowski ${ }^{42}$, L. Chytka ${ }^{117}$, D. Cinca ${ }^{46}$, V. Cindro ${ }^{78}$, I.A. Cioară ${ }^{23}$, A. Ciocio ${ }^{16}$, F. Cirotto ${ }^{106 a, 106 b}$, Z.H. Citron ${ }^{175}$, M. Citterio ${ }^{94 a}$, A. Clark $^{52}$, M.R. Clark $^{38}$, P.J. Clark ${ }^{49}$, R.N. Clarke ${ }^{16}$, C. Clement ${ }^{148 a, 148 b}$, Y. Coadou ${ }^{88}$, M. Cobal ${ }^{167 a, 167 c}$, A. Coccaro ${ }^{53 a, 53 b}$, J. Cochran ${ }^{67}$, A.E.C. Coimbra ${ }^{175}$, L. Colasurdo ${ }^{108}$, B. Cole ${ }^{38}$, A.P. Colijn ${ }^{109}$, J. Collot $^{57}$, P. Conde Muiño ${ }^{128 a, 128 b}$, E. Coniavitis ${ }^{51}$, S.H. Connell ${ }^{147 b}$, I.A. Connelly ${ }^{87}$, S. Constantinescu ${ }^{28 b}$, F. Conventi ${ }^{106 a, l}$, A.M. Cooper-Sarkar ${ }^{122}$, F. Cormier ${ }^{171}$, K.J.R. Cormier ${ }^{161}$, M. Corradi ${ }^{134 a, 134 b}$, 
E.E. Corrigan $^{84}$, F. Corriveau ${ }^{90, m}$, A. Cortes-Gonzalez ${ }^{32}$, M.J. Costa ${ }^{170}$, D. Costanzo ${ }^{141}$, G. Cottin $^{30}$, G. Cowan $^{80}$, B.E. Cox $^{87}$, J. Crane ${ }^{87}$, K. Cranmer ${ }^{112}$, S.J. Crawley ${ }^{56}$,

R.A. Creager ${ }^{124}$, G. Cree $^{31}$, S. Crépé-Renaudin ${ }^{57}$, F. Crescioli ${ }^{83}$, M. Cristinziani ${ }^{23}$, V. Croft ${ }^{112}$, G. Crosetti ${ }^{40 a, 40 b}$, A. Cueto ${ }^{85}$, T. Cuhadar Donszelmann ${ }^{141}$, A.R. Cukierman ${ }^{145}$, M. Curatolo $^{50}$, J. Cúth ${ }^{86}$, S. Czekierda ${ }^{42}$, P. Czodrowski ${ }^{32}$, G. D'amen ${ }^{22 a, 22 b}$, S. D'Auria ${ }^{56}$, L. D'Eramo ${ }^{83}$, M. D'Onofrio ${ }^{77}$, M.J. Da Cunha Sargedas De Sousa ${ }^{36 b, 128 b}$, C. Da Via ${ }^{87}$, W. Dabrowski ${ }^{41 a}$, T. Dado ${ }^{146 a, h}$, S. Dahbi ${ }^{137 e}$, T. Dai ${ }^{92}$, O. Dale ${ }^{15}$, F. Dallaire ${ }^{97}$, C. Dallapiccola ${ }^{89}$, M. Dam ${ }^{39}$, J.R. Dandoy ${ }^{124}$, M.F. Daneri ${ }^{29}$, N.P. Dang ${ }^{176, e}$, N.D Dann ${ }^{87}$, M. Danninger ${ }^{171}$, V. Dao ${ }^{32}$, G. Darbo ${ }^{53 a}$, S. Darmora ${ }^{8}$, O. Dartsi ${ }^{5}$, A. Dattagupta ${ }^{118}$, T. Daubney ${ }^{45}$, W. Davey ${ }^{23}$, C. David ${ }^{45}$, T. Davidek ${ }^{131}$, D.R. Davis $^{48}$, E. Dawe ${ }^{91}$, I. Dawson ${ }^{141}$, K. De $^{8}$, R. de Asmundis ${ }^{106 a}$, A. De Benedetti ${ }^{115}$, S. De Castro ${ }^{22 a, 22 b}$, S. De $\mathrm{Cecco}^{83}$, N. De Groot ${ }^{108}$, P. de Jong ${ }^{109}$, H. De la Torre ${ }^{93}$, F. De Lorenzi ${ }^{67}$, A. De Maria ${ }^{58, n}$, D. De Pedis ${ }^{134 a}$, A. De Salvo ${ }^{134 a}$, U. De Sanctis ${ }^{135 a, 135 b}$, A. De Santo ${ }^{151}$, K. De Vasconcelos Corga ${ }^{88}$, J.B. De Vivie De Regie ${ }^{119}$, C. Debenedetti ${ }^{139}$, D.V. Dedovich ${ }^{68}$, N. Dehghanian ${ }^{3}$, M. Del Gaudio ${ }^{40 a, 40 b}$, J. Del Peso ${ }^{85}$, D. Delgove ${ }^{119}$, F. Deliot ${ }^{138}$, C.M. Delitzsch ${ }^{7}$, A. Dell'Acqua ${ }^{32}$, L. Dell'Asta ${ }^{24}$, M. Della Pietra ${ }^{106 a, 106 b}$, D. della Volpe ${ }^{52}$, M. Delmastro ${ }^{5}$, C. Delporte ${ }^{119}$, P.A. Delsart ${ }^{57}$, D.A. DeMarco ${ }^{161}$, S. Demers ${ }^{179}$, M. Demichev ${ }^{68}$, S.P. Denisov ${ }^{132}$, D. Denysiuk ${ }^{109}$, D. Derendarz ${ }^{42}$, J.E. Derkaoui ${ }^{137 d}$, F. Derue ${ }^{83}$, P. Dervan ${ }^{77}$, K. Desch ${ }^{23}$, C. Deterre ${ }^{45}$, K. Dette ${ }^{161}$, M.R. Devesa ${ }^{29}$, P.O. Deviveiros ${ }^{32}$, A. Dewhurst ${ }^{133}$, S. Dhaliwal ${ }^{25}$, F.A. Di Bello ${ }^{52}$, A. Di Ciaccio ${ }^{135 a, 135 b}$, L. Di Ciaccio ${ }^{5}$, W.K. Di Clemente ${ }^{124}$, C. Di Donato ${ }^{106 a, 106 b}$, A. Di Girolamo ${ }^{32}$, B. Di Micco ${ }^{136 a, 136 b}$, R. Di Nardo ${ }^{32}$, K.F. Di Petrillo ${ }^{59}$, A. Di Simone ${ }^{51}$, R. Di Sipio ${ }^{161}$, D. Di Valentino ${ }^{31}$, C. Diaconu ${ }^{88}$, M. Diamond ${ }^{161}$, F.A. Dias ${ }^{39}$, T. Dias do Vale ${ }^{128 a}$, M.A. Diaz ${ }^{34 a}$, J. Dickinson ${ }^{16}$, E.B. Diehl ${ }^{92}$, J. Dietrich ${ }^{17}$, S. Díez Cornell ${ }^{45}$, A. Dimitrievska ${ }^{16}$, J. Dingfelder ${ }^{23}$, F. Dittus ${ }^{32}$, F. Djama ${ }^{88}$, T. Djobava ${ }^{54 b}$, J.I. Djuvsland ${ }^{60 a}$, M.A.B. do Vale ${ }^{26 c}$, M. Dobre ${ }^{28 b}$, D. Dodsworth ${ }^{25}$, C. Doglioni ${ }^{84}$, J. Dolejsi ${ }^{131}$, Z. Dolezal ${ }^{131}$, M. Donadelli ${ }^{26 d}$, J. Donini ${ }^{37}$, J. Dopke ${ }^{133}$, A. Doria ${ }^{106 a}$, M.T. Dova ${ }^{74}$, A.T. Doyle ${ }^{56}$, E. Drechsler ${ }^{58}$, E. Dreyer ${ }^{144}$, T. Dreyer ${ }^{58}$, M. Dris ${ }^{10}$, Y. Du ${ }^{36 b}$, J. Duarte-Campderros ${ }^{155}$, F. Dubinin ${ }^{98}$, A. Dubreuil ${ }^{52}$, E. Duchovni ${ }^{175}$, G. Duckeck ${ }^{102}$, A. Ducourthial ${ }^{83}$, O.A. Ducu ${ }^{97, o}$, D. Duda ${ }^{109}$, A. Dudarev ${ }^{32}$, A.Chr. Dudder ${ }^{86}$, E.M. Duffield ${ }^{16}$, L. Duflot ${ }^{119}$, M. Dührssen ${ }^{32}$, C. Dülsen ${ }^{177}$, M. Dumancic $^{175}$, A.E. Dumitriu ${ }^{28 b}, p$, A.K. Duncan ${ }^{56}$, M. Dunford ${ }^{60 a}$, A. Duperrin ${ }^{88}$, H. Duran Yildiz ${ }^{4 a}$, M. Düren ${ }^{55}$, A. Durglishvili ${ }^{54 b}$, D. Duschinger ${ }^{47}$, B. Dutta ${ }^{45}$, D. Duvnjak ${ }^{1}$, M. Dyndal $^{45}$, B.S. Dziedzic ${ }^{42}$, C. Eckardtt ${ }^{45}$, K.M. Ecker ${ }^{103}$, R.C. Edgar ${ }^{92}$, T. Eifert ${ }^{32}$, G. Eigen ${ }^{15}$, K. Einsweiler ${ }^{16}$, T. Ekelof ${ }^{168}$, M. El Kacimi ${ }^{137 c}$, R. El Kosseifif ${ }^{88}$, V. Ellajosyula ${ }^{88}$, M. Ellert $^{168}$, F. Ellinghaus ${ }^{177}$, A.A. Elliot ${ }^{172}$, N. Ellis $^{32}$, J. Elmsheuser ${ }^{27}$, M. Elsing ${ }^{32}$, D. Emeliyanov ${ }^{133}$, Y. Enari ${ }^{157}$, J.S. Ennis ${ }^{173}$, M.B. Epland ${ }^{48}$, J. Erdmann ${ }^{46}$, A. Ereditato ${ }^{18}$, S. Errede ${ }^{169}$, M. Escalier ${ }^{119}$, C. Escobar ${ }^{170}$, B. Esposito ${ }^{50}$, O. Estrada Pastor ${ }^{170}$, A.I. Etienvre ${ }^{138}$, E. Etzion ${ }^{155}$, H. Evans $^{64}$, A. Ezhilov ${ }^{125}$, M. Ezzi ${ }^{137 e}$, F. Fabbri ${ }^{22 a, 22 b}$, L. Fabbri ${ }^{22 a, 22 b}$, V. Fabiani ${ }^{108}$, G. Facini ${ }^{81}$, R.M. Faisca Rodrigues Pereira ${ }^{128 a}$, R.M. Fakhrutdinov ${ }^{132}$, S. Falciano ${ }^{134 a}$, P.J. Falke ${ }^{5}$, S. Falke ${ }^{5}$, J. Faltova ${ }^{131}$, Y. Fang ${ }^{35 a}$, M. Fanti ${ }^{94 a, 94 b}$, A. Farbin ${ }^{8}$, A. Farilla ${ }^{136 a}$, E.M. Farina ${ }^{123 a, 123 b}$, T. Farooque ${ }^{93}$, S. Farrell ${ }^{16}$, S.M. Farrington ${ }^{173}$, P. Farthouat ${ }^{32}$, F. Fassi ${ }^{137 e}$, P. Fassnacht ${ }^{32}$, D. Fassouliotis ${ }^{9}$, M. Faucci Giannelli ${ }^{49}$, A. Favareto ${ }^{53 a, 53 b}$, W.J. Fawcett ${ }^{52}$, L. Fayard ${ }^{119}$, O.L. Fedin ${ }^{125, q}$, W. Fedorko ${ }^{171}$, M. Feickert ${ }^{43}$, S. Feigl ${ }^{121}$, L. Feligioni ${ }^{88}$, C. Feng ${ }^{36 b}$, E.J. Feng ${ }^{32}$, M. Feng ${ }^{48}$, M.J. Fenton ${ }^{56}$, A.B. Fenyuk ${ }^{132}$, L. Feremenga ${ }^{8}$, J. Ferrando ${ }^{45}$, A. Ferrari ${ }^{168}$, P. Ferrari ${ }^{109}$, R. Ferrari ${ }^{123 a}$, D.E. Ferreira de Lima ${ }^{60 b}$, A. Ferrer ${ }^{170}$, D. Ferrere ${ }^{52}$, C. Ferretti ${ }^{92}$, F. Fiedler ${ }^{86}$, A. Filipčic ${ }^{78}$, F. Filthaut ${ }^{108}$, M. Fincke-Keeler ${ }^{172}$, K.D. Finelli ${ }^{24}$, M.C.N. Fiolhais ${ }^{128 a, 128 c, r}$, L. Fiorini ${ }^{170}$, C. Fischer ${ }^{13}$, J. Fischer ${ }^{177}$, W.C. Fisher ${ }^{93}$, N. Flaschel ${ }^{45}$, I. Fleck ${ }^{143}$, P. Fleischmann ${ }^{92}$, R.R.M. Fletcher ${ }^{124}$, T. Flick ${ }^{177}$, B.M. Flierl ${ }^{102}$, L.M. Flores ${ }^{124}$, L.R. Flores Castillo ${ }^{62 a}$, N. Fomin ${ }^{15}$, G.T. Forcolin ${ }^{87}$, A. Formica ${ }^{138}$, F.A. Förster ${ }^{13}$, A.C. Forti ${ }^{87}$, 
A.G. Foster ${ }^{19}$, D. Fournier ${ }^{119}$, H. Fox ${ }^{75}$, S. Fracchia ${ }^{141}$, P. Francavilla ${ }^{126 a, 126 b}$, M. Franchini ${ }^{22 a, 22 b}$, S. Franchino ${ }^{60 a}$, D. Francis ${ }^{32}$, L. Franconi ${ }^{121}$, M. Franklin ${ }^{59}$, M. Frate ${ }^{166}$, M. Fraternali ${ }^{123 a, 123 b}$, D. Freeborn ${ }^{81}$, S.M. Fressard-Batraneanu ${ }^{32}$, B. Freund ${ }^{97}$, W.S. Freund ${ }^{26 a}$, D. Froidevaux ${ }^{32}$, J.A. Frost ${ }^{122}$, C. Fukunaga ${ }^{158}$, T. Fusayasu ${ }^{104}$, J. Fuster ${ }^{170}$, O. Gabizon ${ }^{154}$, A. Gabrielli ${ }^{22 a, 22 b}$, A. Gabrielli ${ }^{16}$, G.P. Gach ${ }^{41 a}$, S. Gadatsch ${ }^{52}$, S. Gadomski ${ }^{80}$, P. Gadow ${ }^{103}$, G. Gagliardi ${ }^{53 a, 53 b}$, L.G. Gagnon ${ }^{97}$, C. Galea ${ }^{28 b}$, B. Galhardo ${ }^{128 a, 128 c}$, E.J. Gallas ${ }^{122}$, B.J. Gallop ${ }^{133}$, P. Gallus ${ }^{130}$, G. Galster ${ }^{39}$, R. Gamboa Goni ${ }^{79}$, K.K. Gan ${ }^{113}$, S. Ganguly ${ }^{175}$, Y. Gao ${ }^{77}$, Y.S. Gao ${ }^{145, f}$, F.M. Garay Walls ${ }^{34 a}$, C. García ${ }^{170}$, J.E. García Navarro ${ }^{170}$, J.A. García Pascual ${ }^{35 a}$, M. Garcia-Sciveres ${ }^{16}$, R.W. Gardner ${ }^{33}$, N. Garelli ${ }^{145}$, V. Garonne ${ }^{121}$, K. Gasnikova ${ }^{45}$, A. Gaudiello $53 a, 53 b$, G. Gaudio ${ }^{123 a}$, I.L. Gavrilenko ${ }^{98}$, A. Gavrilyuk ${ }^{99}$, C. Gay ${ }^{171}$, G. Gaycken ${ }^{23}$, E.N. Gazis ${ }^{10}$, C.N.P. Gee ${ }^{133}$, J. Geisen ${ }^{58}$, M. Geisen ${ }^{86}$, M.P. Geisler ${ }^{60 a}$, K. Gellerstedt ${ }^{148 a, 148 b}$, C. Gemme ${ }^{53 a}$, M.H. Genest ${ }^{57}$, C. Geng ${ }^{92}$, S. Gentile ${ }^{134 a, 134 b}$, C. Gentsos ${ }^{156}$, S. George ${ }^{80}$, D. Gerbaudo ${ }^{13}$, G. Gessner ${ }^{46}$, S. Ghasemi ${ }^{143}$, M. Ghneimat ${ }^{23}$, B. Giacobbe ${ }^{22 a}$, S. Giagu ${ }^{134 a, 134 b}$, N. Giangiacomi ${ }^{22 a}$,22b, P. Giannetti ${ }^{126 a}$, S.M. Gibson ${ }^{80}$, M. Gignac ${ }^{139}$, D. Gillberg ${ }^{31}$, G. Gilles ${ }^{177}$, D.M. Gingrich ${ }^{3, d}$, M.P. Giordani ${ }^{167 a, 167 c}$, F.M. Giorgi ${ }^{22 a}$, P.F. Giraud ${ }^{138}$, P. Giromini ${ }^{59}$, G. Giugliarelli ${ }^{167 a, 167 c}$, D. Giugni ${ }^{94 a}$, F. Giuli ${ }^{122}$, M. Giulini ${ }^{60 b}$, S. Gkaitatzis ${ }^{156}$, I. Gkialas ${ }^{9, s}$, E.L. Gkougkousis ${ }^{13}$, P. Gkountoumis ${ }^{10}$, L.K. Gladilin ${ }^{101}$, C. Glasman ${ }^{85}$, J. Glatzer ${ }^{13}$, P.C.F. Glaysher ${ }^{45}$, A. Glazov ${ }^{45}$, M. Goblirsch-Kolb ${ }^{25}$, J. Godlewski ${ }^{42}$, S. Goldfarb ${ }^{91}$, T. Golling ${ }^{52}$, D. Golubkov ${ }^{132}$, A. Gomes ${ }^{128 a, 128 b, 128 e}$, R. Gonçalo ${ }^{128 a}$, R. Goncalves Gama ${ }^{26 b}$, G. Gonella ${ }^{51}$, L. Gonella ${ }^{19}$, A. Gongadze ${ }^{68}$, F. Gonnella ${ }^{19}$, J.L. Gonski ${ }^{59}$, S. González de la Hoz ${ }^{170}$, S. Gonzalez-Sevilla ${ }^{52}$, L. Goossens ${ }^{32}$, P.A. Gorbounov ${ }^{99}$, H.A. Gordon ${ }^{27}$, B. Gorini ${ }^{32}$, E. Gorini ${ }^{76 a}$,76b, A. Gorišek ${ }^{78}$, A.T. Goshaw ${ }^{48}$, C. Gössling ${ }^{46}$, M.I. Gostkin ${ }^{68}$, C.A. Gottardo ${ }^{23}$, C.R. Goudet ${ }^{119}$, D. Goujdami ${ }^{137 c}$, A.G. Goussiou ${ }^{140}$, N. Govender ${ }^{147 b, t}$, C. Goy ${ }^{5}$, E. Gozani ${ }^{154}$, I. Grabowska-Bold ${ }^{41 a}$, P.O.J. Gradin ${ }^{168}$, E.C. Graham ${ }^{77}$, J. Gramling ${ }^{166}$, E. Gramstad ${ }^{121}$, S. Grancagnolo ${ }^{17}$, V. Gratchev ${ }^{125}$, P.M. Gravila ${ }^{28 f}$, C. Gray ${ }^{56}$, H.M. Gray ${ }^{16}$, Z.D. Greenwood ${ }^{82, u}$, C. Grefe $^{23}$, K. Gregersen ${ }^{81}$, I.M. Gregor ${ }^{45}$, P. Grenier ${ }^{145}$, K. Grevtsov ${ }^{45}$, J. Griffiths ${ }^{8}$, A.A. Grillo ${ }^{139}$, K. Grimm ${ }^{145}$, S. Grinstein ${ }^{13, v}$, Ph. Gris ${ }^{37}$, J.-F. Grivaz ${ }^{119}$, S. Groh ${ }^{86}$, E. Gross ${ }^{175}$, J. Grosse-Knetter ${ }^{58}$, G.C. Grossi ${ }^{82}$, Z.J. Grout ${ }^{81}$, A. Grummer ${ }^{107}$, L. Guan ${ }^{92}$, W. Guan ${ }^{176}$, J. Guenther ${ }^{32}$, A. Guerguichon ${ }^{119}$, F. Guescini ${ }^{163 a}$, D. Guest ${ }^{166}$, O. Gueta ${ }^{155}$, R. Gugel ${ }^{51}$, B. Gui ${ }^{113}$, T. Guillemin ${ }^{5}$, S. Guindon ${ }^{32}$, U. Gul ${ }^{56}$, C. Gumpert ${ }^{32}$, J. Guo ${ }^{36 c}$, W. Guo ${ }^{92}$,

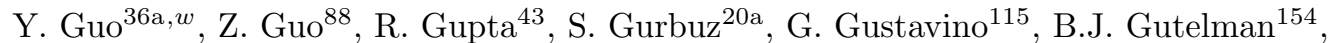
P. Gutierrez ${ }^{115}$, N.G. Gutierrez Ortiz ${ }^{81}$, C. Gutschow ${ }^{81}$, C. Guyot ${ }^{138}$, M.P. Guzik ${ }^{41 a}$, C. Gwenlan ${ }^{122}$, C.B. Gwilliam ${ }^{77}$, A. Hönle ${ }^{103}$, A. Haas ${ }^{112}$, C. Haber ${ }^{16}$, H.K. Hadavand ${ }^{8}$,

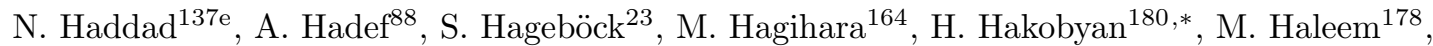
J. Haley ${ }^{116}$, G. Halladjian ${ }^{93}$, G.D. Hallewell ${ }^{88}$, K. Hamacher ${ }^{177}$, P. Hamal ${ }^{117}$, K. Hamano ${ }^{172}$, A. Hamilton ${ }^{147 a}$, G.N. Hamity ${ }^{141}$, K. $\operatorname{Han}^{36 a, x}$, L. Han ${ }^{36 a}$, S. Han ${ }^{35 a, 35 d}$, K. Hanagaki ${ }^{69, y}$, M. Hance ${ }^{139}$, D.M. Handl ${ }^{102}$, B. Haney ${ }^{124}$, R. Hankache ${ }^{83}$, P. Hanke ${ }^{60 a}$, E. Hansen ${ }^{84}$,

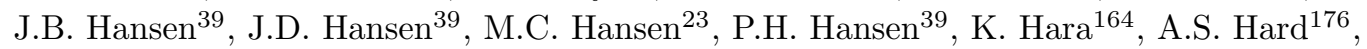
T. Harenberg ${ }^{177}$, S. Harkusha ${ }^{95}$, P.F. Harrison ${ }^{173}$, N.M. Hartmann ${ }^{102}$, Y. Hasegawa ${ }^{142}$, A. Hasib ${ }^{49}$, S. Hassani ${ }^{138}$, S. Haug ${ }^{18}$, R. Hauser ${ }^{93}$, L. Hauswald ${ }^{47}$, L.B. Havener ${ }^{38}$, M. Havranek ${ }^{130}$, C.M. Hawkes ${ }^{19}$, R.J. Hawkings ${ }^{32}$, D. Hayden ${ }^{93}$, C. Hayes ${ }^{150}$, C.P. Hays ${ }^{122}$, J.M. Hays ${ }^{79}$, H.S. Hayward ${ }^{77}$, S.J. Haywood ${ }^{133}$, M.P. Heath ${ }^{49}$, V. Hedberg ${ }^{84}$, L. Heelan ${ }^{8}$, S. Heer ${ }^{23}$, K.K. Heidegger ${ }^{51}$, J. Heilman ${ }^{31}$, S. Heim ${ }^{45}$, T. Heim ${ }^{16}$, B. Heinemann ${ }^{45, z}$, J.J. Heinrich ${ }^{102}$, L. Heinrich ${ }^{112}$, C. Heinz ${ }^{55}$, J. Hejbal ${ }^{129}$, L. Helary ${ }^{32}$, A. Held ${ }^{171}$, S. Hellesund ${ }^{121}$, S. Hellman ${ }^{148 a, 148 b}$, C. Helsens ${ }^{32}$, R.C.W. Henderson ${ }^{75}$, Y. Heng ${ }^{176}$, S. Henkelmann ${ }^{171}$, A.M. Henriques Correia ${ }^{32}$, G.H. Herbert ${ }^{17}$, H. Herde ${ }^{25}$, V. Herget ${ }^{178}$, Y. Hernández Jiménez ${ }^{147 c}$, H. Herr ${ }^{86}$, G. Herten ${ }^{51}$, R. Hertenberger ${ }^{102}$, L. Hervas ${ }^{32}$, T.C. Herwig ${ }^{124}$, G.G. Hesketh ${ }^{81}$, N.P. Hessey ${ }^{163 a}$, J.W. Hetherly ${ }^{43}$, S. Higashino ${ }^{69}$, E. Higón-Rodriguez ${ }^{170}$, K. Hildebrand ${ }^{33}$, 
E. Hill ${ }^{172}$, J.C. Hill ${ }^{30}$, K.H. Hiller ${ }^{45}$, S.J. Hillier ${ }^{19}$, M. Hils ${ }^{47}$, I. Hinchliffe ${ }^{16}$, M. Hirose ${ }^{120}$, D. Hirschbuehl ${ }^{177}$, B. Hiti ${ }^{78}$, O. Hladik ${ }^{129}$, D.R. Hlaluku ${ }^{147 c}$, X. Hoad ${ }^{49}$, J. Hobbs ${ }^{150}$, N. Hod ${ }^{163 a}$, M.C. Hodgkinson ${ }^{141}$, A. Hoecker ${ }^{32}$, M.R. Hoeferkamp ${ }^{107}$, F. Hoenig ${ }^{102}$, D. Hohn ${ }^{23}$, D. Hohov ${ }^{119}$, T.R. Holmes ${ }^{33}$, M. Holzbock ${ }^{102}$, M. Homann ${ }^{46}$, S. Honda ${ }^{164}$, T. Honda ${ }^{69}$, T.M. Hong ${ }^{127}$, B.H. Hooberman ${ }^{169}$, W.H. Hopkins ${ }^{118}$, Y. Horii ${ }^{105}$, P. Horn ${ }^{47}$, A.J. Horton ${ }^{144}$, L.A. Horyn ${ }^{33}$,

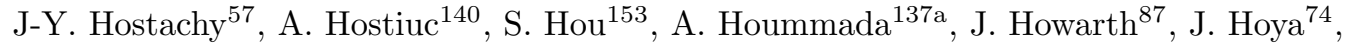
M. Hrabovsky ${ }^{117}$, J. Hrdinka ${ }^{32}$, I. Hristova ${ }^{17}$, J. Hrivnac ${ }^{119}$, T. Hryn'ova ${ }^{5}$, A. Hrynevich ${ }^{96}$, P.J. Hsu ${ }^{63}$, S.-C. Hsu ${ }^{140}$, Q. Hu ${ }^{27}$, S. Hu ${ }^{36 c}$, Y. Huang ${ }^{35 a}$, Z. Hubacek ${ }^{130}$, F. Hubaut ${ }^{88}$, M. Huebner ${ }^{23}$, F. Huegging ${ }^{23}$, T.B. Huffman ${ }^{122}$, E.W. Hughes ${ }^{38}$, M. Huhtinen ${ }^{32}$, R.F.H. Hunter ${ }^{31}$,

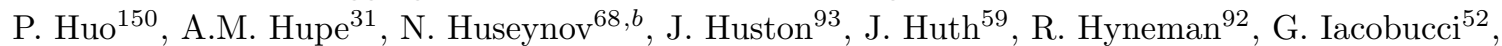
G. Iakovidis ${ }^{27}$, I. Ibragimov ${ }^{143}$, L. Iconomidou-Fayard ${ }^{119}$, Z. Idrissi ${ }^{137 e}$, P. Iengo ${ }^{32}$, R. Ignazzi $^{39}$, O. Igonkina ${ }^{109, a a}$, R. Iguchi ${ }^{157}$, T. Iizawa ${ }^{174}$, Y. Ikegami ${ }^{69}$, M. Ikeno ${ }^{69}$, D. Iliadis ${ }^{156}$, N. Ilic ${ }^{145}$,

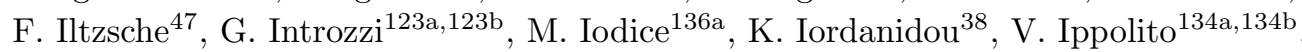

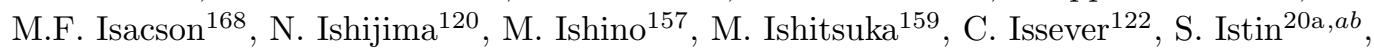
F. Ito ${ }^{164}$, J.M. Iturbe Ponce ${ }^{62 a}$, R. Iuppa ${ }^{162 a, 162 b}$, A. Ivina ${ }^{175}$, H. Iwasaki ${ }^{69}$, J.M. Izen ${ }^{44}$, V. Izzo ${ }^{106 a}$, S. Jabbar ${ }^{3}$, P. Jacka ${ }^{129}$, P. Jackson ${ }^{1}$, R.M. Jacobs ${ }^{23}$, V. Jain ${ }^{2}$, G. Jäkel ${ }^{177}$, K.B. Jakobi ${ }^{86}$, K. Jakobs ${ }^{51}$, S. Jakobsen ${ }^{65}$, T. Jakoubek ${ }^{129}$, D.O. Jamin ${ }^{116}$, D.K. Jana ${ }^{82}$, R. Jansky ${ }^{52}$, J. Janssen ${ }^{23}$, M. Janus ${ }^{58}$, P.A. Janus ${ }^{41 a}$, G. Jarlskog ${ }^{84}$, N. Javadov ${ }^{68, b}$, T. Javůrek ${ }^{51}$, M. Javurkova ${ }^{51}$, F. Jeanneau ${ }^{138}$, L. Jeanty ${ }^{16}$, J. Jejelava ${ }^{54 a, a c}$, A. Jelinskas ${ }^{173}$, P. Jenni ${ }^{51, a d}$, J. Jeong ${ }^{45}$, C. Jeske ${ }^{173}$, S. Jézéquel ${ }^{5}$, H. Ji ${ }^{176}$, J. Jia ${ }^{150}$, H. Jiang ${ }^{67}$, Y. Jiang ${ }^{36 a}$, Z. Jiang ${ }^{145}$, S. Jiggins ${ }^{51}$, F.A. Jimenez Morales ${ }^{37}$, J. Jimenez Pena ${ }^{170}$, S. Jin ${ }^{35 b}$, A. Jinaru ${ }^{28 b}$, O. Jinnouchi ${ }^{159}$, H. Jivan ${ }^{147 c}$, P. Johansson ${ }^{141}$, K.A. Johns ${ }^{7}$, C.A. Johnson ${ }^{64}$, W.J. Johnson ${ }^{140}$, K. Jon-And ${ }^{148 a, 148 b}$, R.W.L. Jones ${ }^{75}$, S.D. Jones ${ }^{151}$, S. Jones ${ }^{7}$, T.J. Jones ${ }^{77}$, J. Jongmanns ${ }^{60 a}$, P.M. Jorge ${ }^{128 \mathrm{a}, 128 \mathrm{~b}}$, J. Jovicevic ${ }^{163 a}$, X. Ju ${ }^{176}$, J.J. Junggeburth ${ }^{103}$, A. Juste Rozas ${ }^{13, v}$, A. Kaczmarska ${ }^{42}$, M. Kado ${ }^{119}$, H. Kagan ${ }^{113}$, M. Kagan ${ }^{145}$, T. Kaji ${ }^{174}$, E. Kajomovitz ${ }^{154}$, C.W. Kalderon ${ }^{84}$, A. Kaluza ${ }^{86}$, S. Kama ${ }^{43}$, A. Kamenshchikov ${ }^{132}$, L. Kanjir ${ }^{78}$, Y. Kano ${ }^{157}$, V.A. Kantserov ${ }^{100}$, J. Kanzaki ${ }^{69}$, B. Kaplan ${ }^{112}$, L.S. Kaplan ${ }^{176}$, D. Kar ${ }^{147 c}$, M.J. Kareem ${ }^{163 b}$, E. Karentzos ${ }^{10}$, S.N. Karpov ${ }^{68}$, Z.M. Karpova ${ }^{68}$, V. Kartvelishvili ${ }^{75}$, A.N. Karyukhin ${ }^{132}$, K. Kasahara ${ }^{164}$, L. Kashif ${ }^{176}$, R.D. Kass ${ }^{113}$, A. Kastanas ${ }^{149}$, Y. Kataoka ${ }^{157}$, C. Kato ${ }^{157}$, A. Katre ${ }^{52}$, J. Katzy ${ }^{45}$, K. Kawade ${ }^{70}$, K. Kawagoe ${ }^{73}$, T. Kawamoto ${ }^{157}$, G. Kawamura ${ }^{58}$, E.F. Kay ${ }^{77}$, V.F. Kazanin ${ }^{111, c}$, R. Keeler ${ }^{172}$, R. Kehoe ${ }^{43}$, J.S. Keller ${ }^{31}$, E. Kellermann ${ }^{84}$, J.J. Kempster ${ }^{19}$, J. Kendrick ${ }^{19}$, O. Kepka ${ }^{129}$, B.P. Kerševan ${ }^{78}$, S. Kersten ${ }^{177}$, R.A. Keyes ${ }^{90}$, M. Khader ${ }^{169}$, F. Khalil-zada ${ }^{12}$, A. Khanov ${ }^{116}$, A.G. Kharlamov ${ }^{111, c}$, T. Kharlamova ${ }^{111}$, A. Khodinov ${ }^{160}$, T.J. Khoo ${ }^{52}$, V. Khovanskiy ${ }^{99, *}$, E. Khramov ${ }^{68}$, J. Khubua ${ }^{54 b, a e}$, S. Kido ${ }^{70}$, M. Kiehn ${ }^{52}$, C.R. Kilby ${ }^{80}$, H.Y. Kim ${ }^{8}$, S.H. Kim ${ }^{164}$, Y.K. Kim ${ }^{33}$, N. Kimura ${ }^{167 a, 167 c}$, O.M. Kind ${ }^{17}$, B.T. King ${ }^{77}$, D. Kirchmeier ${ }^{47}$, J. Kirk ${ }^{133}$, A.E. Kiryunin ${ }^{103}$, T. Kishimoto ${ }^{157}$, D. Kisielewska ${ }^{41 a}$, V. Kitali ${ }^{45}$, O. Kivernyk ${ }^{5}$, E. Kladiva ${ }^{146 \mathrm{~b}, *}$, T. Klapdor-Kleingrothaus ${ }^{51}$, M.H. Klein ${ }^{92}$, M. Klein ${ }^{77}$, U. Klein ${ }^{77}$, K. Kleinknecht ${ }^{86}$, P. Klimek ${ }^{110}$, A. Klimentov ${ }^{27}$, R. Klingenberg ${ }^{46, *}$, T. Klingl ${ }^{23}$, T. Klioutchnikova ${ }^{32}$, F.F. Klitzner ${ }^{102}$, P. Kluit ${ }^{109}$, S. Kluth ${ }^{103}$, E. Kneringer ${ }^{65}$, E.B.F.G. Knoops ${ }^{88}$, A. Knue ${ }^{51}$, A. Kobayashi ${ }^{157}$, D. Kobayashi ${ }^{73}$, T. Kobayashi ${ }^{157}$, M. Kobel ${ }^{47}$, M. Kocian ${ }^{145}$, P. Kodys ${ }^{131}$, T. Koffas ${ }^{31}$, E. Koffeman ${ }^{109}$, N.M. Köhler ${ }^{103}$, T. Koi ${ }^{145}$, M. Kolb ${ }^{60 b}$, I. Koletsou ${ }^{5}$, T. Kondo ${ }^{69}$, N. Kondrashova ${ }^{36 c}$, K. Köneke ${ }^{51}$, A.C. König ${ }^{108}$, T. Kono ${ }^{69, a f}$, R. Konoplich ${ }^{112, a g}$, N. Konstantinidis ${ }^{81}$, B. Konya ${ }^{84}$, R. Kopeliansky ${ }^{64}$, S. Koperny ${ }^{41 a}$, K. Korcyl ${ }^{42}$, K. Kordas ${ }^{156}$, A. Korn ${ }^{81}$, I. Korolkov ${ }^{13}$, E.V. Korolkova ${ }^{141}$, O. Kortner ${ }^{103}$, S. Kortner ${ }^{103}$, T. Kosek ${ }^{131}$, V.V. Kostyukhin ${ }^{23}$, A. Kotwal ${ }^{48}$, A. Koulouris ${ }^{10}$,

A. Kourkoumeli-Charalampidi ${ }^{123 a, 123 b}$, C. Kourkoumelis ${ }^{9}$, E. Kourlitis ${ }^{141}$, V. Kouskoura ${ }^{27}$, A.B. Kowalewska ${ }^{42}$, R. Kowalewski ${ }^{172}$, T.Z. Kowalski ${ }^{41 a}$, C. Kozakai ${ }^{157}$, W. Kozanecki ${ }^{138}$, A.S. Kozhin ${ }^{132}$, V.A. Kramarenko ${ }^{101}$, G. Kramberger ${ }^{78}$, D. Krasnopevtsev ${ }^{100}$, M.W. Krasny ${ }^{83}$, 
A. Krasznahorkay ${ }^{32}$, D. Krauss ${ }^{103}$, J.A. Kremer ${ }^{41 a}$, J. Kretzschmar ${ }^{77}$, K. Kreutzfeldt ${ }^{55}$, P. Krieger ${ }^{161}$, K. Krizka ${ }^{16}$, K. Kroeninger ${ }^{46}$, H. Kroha ${ }^{103}$, J. Kroll ${ }^{129}$, J. Kroll ${ }^{124}$, J. Kroseberg ${ }^{23}$, J. Krstic ${ }^{14}$, U. Kruchonak ${ }^{68}$, H. Krüger ${ }^{23}$, N. Krumnack ${ }^{67}$, M.C. Kruse ${ }^{48}$, T. Kubota ${ }^{91}$, S. Kuday ${ }^{4 b}$, J.T. Kuechler ${ }^{177}$, S. Kuehn ${ }^{32}$, A. Kugel ${ }^{60 a}$, F. Kuger ${ }^{178}$, T. Kuhl ${ }^{45}$, V. Kukhtin ${ }^{68}$, R. Kukla ${ }^{88}$, Y. Kulchitsky ${ }^{95}$, S. Kuleshov ${ }^{34 b}$, Y.P. Kulinich ${ }^{169}$, M. Kuna ${ }^{57}$, T. Kunigo ${ }^{71}$, A. Kupco ${ }^{129}$, T. Kupfer ${ }^{46}$, O. Kuprash ${ }^{155}$, H. Kurashige ${ }^{70}$, L.L. Kurchaninov ${ }^{163 a}$,

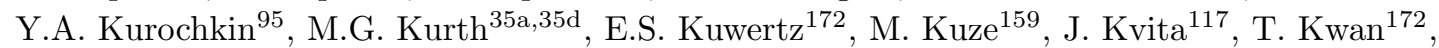
A. La Rosa ${ }^{103}$, J.L. La Rosa Navarro ${ }^{26 \mathrm{~d}}$, L. La Rotonda ${ }^{40 a, 40 b}$, F. La Ruffa ${ }^{40 a, 40 b}$, C. Lacasta ${ }^{170}$, F. Lacava ${ }^{134 a, 134 b}$, J. Lacey ${ }^{45}$, D.P.J. Lack ${ }^{87}$, H. Lacker ${ }^{17}$, D. Lacour ${ }^{83}$, E. Ladygin ${ }^{68}$, R. Lafaye ${ }^{5}$, B. Laforge ${ }^{83}$, S. Lai ${ }^{58}$, S. Lammers ${ }^{64}$, W. Lampl ${ }^{7}$, E. Lançon ${ }^{27}$, U. Landgraf ${ }^{51}$, M.P.J. Landon ${ }^{79}$, M.C. Lanfermann ${ }^{52}$, V.S. Lang ${ }^{45}$, J.C. Lange ${ }^{13}$, R.J. Langenberg ${ }^{32}$, A.J. Lankford ${ }^{166}$, F. Lanni ${ }^{27}$, K. Lantzsch ${ }^{23}$, A. Lanza ${ }^{123 a}$, A. Lapertosa ${ }^{53 a, 53 b}$, S. Laplace ${ }^{83}$, J.F. Laporte ${ }^{138}$, T. Lari ${ }^{94 a}$, F. Lasagni Manghi ${ }^{22 a, 22 b}$, M. Lassnig ${ }^{32}$, T.S. Lau ${ }^{62 a}$, A. Laudrain ${ }^{119}$, A.T. Law ${ }^{139}$, P. Laycock ${ }^{77}$, M. Lazzaroni ${ }^{94 a, 94 b}$, B. Le ${ }^{91}$, O. Le Dortz ${ }^{83}$, E. Le Guirriec ${ }^{88}$, E.P. Le Quilleuc ${ }^{138}$, M. LeBlanc ${ }^{7}$, T. LeCompte ${ }^{6}$, F. Ledroit-Guillon ${ }^{57}$, C.A. Lee ${ }^{27}$, G.R. Lee ${ }^{34 a}$, S.C. Lee ${ }^{153}$, L. Lee ${ }^{59}$, B. Lefebvre ${ }^{90}$, M. Lefebvre ${ }^{172}$, F. Legger ${ }^{102}$, C. Leggett ${ }^{16}$, G. Lehmann Miotto ${ }^{32}$, W.A. Leight ${ }^{45}$, A. Leisos ${ }^{156, a h}$, M.A.L. Leite ${ }^{26 \mathrm{~d}}$, R. Leitner ${ }^{131}$, D. Lellouch ${ }^{175}$, B. Lemmer ${ }^{58}$, K.J.C. Leney ${ }^{81}$, T. Lenz ${ }^{23}$, B. Lenzi ${ }^{32}$, R. Leone ${ }^{7}$, S. Leone ${ }^{126 a}$, C. Leonidopoulos ${ }^{49}$, G. Lerner ${ }^{151}$, C. Leroy ${ }^{97}$, R. Les $^{161}$, A.A.J. Lesage ${ }^{138}$, C.G. Lester ${ }^{30}$, M. Levchenko ${ }^{125}$, J. Levêque ${ }^{5}$, D. Levin ${ }^{92}$, L.J. Levinson ${ }^{175}$, D. Lewis ${ }^{79}$, B. Li ${ }^{36 a}, w$, C.-Q. $\mathrm{Li}^{36 a}, \mathrm{H}^{3} \mathrm{Li}^{36 \mathrm{~b}}, \mathrm{~L} . \mathrm{Li}^{36 c}, \mathrm{Q}^{3} \mathrm{Li}^{35 \mathrm{a}, 35 \mathrm{~d}}$, Q. $\mathrm{Li}^{36 \mathrm{a}}$,

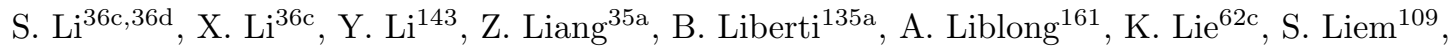
A. Limosani ${ }^{152}$, C.Y. $\operatorname{Lin}^{30}$, K. $\operatorname{Lin}^{93}$, S.C. $\operatorname{Lin}^{182}$, T.H. $\operatorname{Lin}^{86}$, R.A. Linck ${ }^{64}$, B.E. Lindquist ${ }^{150}$, A.L. Lionti ${ }^{52}$, E. Lipeles ${ }^{124}$, A. Lipniacka ${ }^{15}$, M. Lisovyi ${ }^{60 b}$, T.M. Liss ${ }^{169, a i}$, A. Lister ${ }^{171}$,

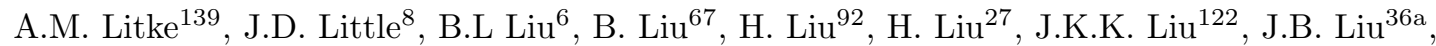

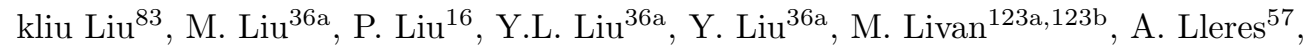

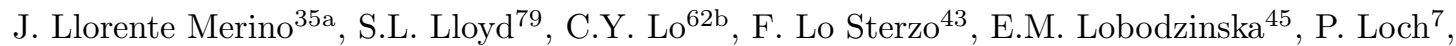
F.K. Loebinger ${ }^{87}$, A. Loesle ${ }^{51}$, K.M. Loew ${ }^{25}$, T. Lohse $^{17}$, K. Lohwasser ${ }^{141}$, M. Lokajicek ${ }^{129}$, B.A. Long ${ }^{24}$, J.D. Long ${ }^{169}$, R.E. Long ${ }^{75}$, L. Longo ${ }^{76 a, 76 b}$, K.A. Looper ${ }^{113}$, J.A. Lopez ${ }^{34 b}$, I. Lopez Paz ${ }^{13}$, A. Lopez Solis ${ }^{83}$, J. Lorenz ${ }^{102}$, N. Lorenzo Martinez ${ }^{5}$, M. Losada ${ }^{21}$, P.J. Lösel ${ }^{102}$,

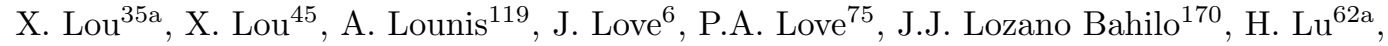
N. $\mathrm{Lu}^{92}$, Y.J. Lu ${ }^{63}$, H.J. Lubatti ${ }^{140}$, C. Luci ${ }^{134 a, 134 b}$, A. Lucotte ${ }^{57}$, C. Luedtke ${ }^{51}$, F. Luehring ${ }^{64}$, I. Luise ${ }^{83}$, W. Lukas $^{65}$, L. Luminari ${ }^{134 a}$, B. Lund-Jensen ${ }^{149}$, M.S. Lutz ${ }^{89}$, P.M. Luzi ${ }^{83}$, D. Lynn ${ }^{27}$, R. Lysak ${ }^{129}$, E. Lytken ${ }^{84}$, F. Lyu ${ }^{35 a}$, V. Lyubushkin ${ }^{68}$, H. Ma ${ }^{27}$, L.L. Ma ${ }^{36 \mathrm{~b}}$, Y. Ma ${ }^{36 \mathrm{~b}}$, G. Maccarrone ${ }^{50}$, A. Macchiolo ${ }^{103}$, C.M. Macdonald ${ }^{141}$, B. Maček ${ }^{78}$, J. Machado Miguens ${ }^{124}$, D. Madaffari ${ }^{170}$, R. Madar ${ }^{37}$, W.F. Mader ${ }^{47}$, A. Madsen ${ }^{45}$, N. Madysa ${ }^{47}$, J. Maeda ${ }^{70}$, S. Maeland ${ }^{15}$, T. Maeno ${ }^{27}$, A.S. Maevskiy ${ }^{101}$, V. Magerl ${ }^{51}$, C. Maidantchik ${ }^{26 a}$, T. Maier ${ }^{102}$, A. Maio ${ }^{128 a, 128 b, 128 e}$, O. Majersky ${ }^{146 a}$, S. Majewski ${ }^{118}$, Y. Makida $^{69}$, N. Makovec ${ }^{119}$, B. Malaescu ${ }^{83}$, Pa. Malecki ${ }^{42}$, V.P. Maleev ${ }^{125}$, F. Malek ${ }^{57}$, U. Mallik ${ }^{66}$, D. Malon ${ }^{6}$, C. Malone ${ }^{30}$, S. Maltezos ${ }^{10}$, S. Malyukov ${ }^{32}$, J. Mamuzic ${ }^{170}$, G. Mancini ${ }^{50}$, I. Mandić ${ }^{78}$, J. Maneira ${ }^{128 a, 128 b}$, L. Manhaes de Andrade Filho ${ }^{26 \mathrm{~b}}$, J. Manjarres Ramos ${ }^{47}$, K.H. Mankinen ${ }^{84}$, A. Mann ${ }^{102}$, A. Manousos ${ }^{65}$, B. Mansoulie ${ }^{138}$, J.D. Mansour ${ }^{35 a}$, R. Mantifel ${ }^{90}$, M. Mantoani ${ }^{58}$, S. Manzoni ${ }^{94 a, 94 b}$, G. Marceca ${ }^{29}$, L. March $^{52}$, L. Marchese ${ }^{122}$, G. Marchiori ${ }^{83}$, M. Marcisovsky ${ }^{129}$, C.A. Marin Tobon ${ }^{32}$, M. Marjanovic ${ }^{37}$, D.E. Marley ${ }^{92}$, F. Marroquim ${ }^{26 a}$, Z. Marshall ${ }^{16}$, M.U.F Martensson ${ }^{168}$, S. Marti-Garcia ${ }^{170}$, C.B. Martin ${ }^{113}$, T.A. Martin ${ }^{173}$, V.J. Martin ${ }^{49}$, B. Martin dit Latour ${ }^{15}$, M. Martinez ${ }^{13, v}$, V.I. Martinez Outschoorn ${ }^{89}$, S. Martin-Haugh ${ }^{133}$, V.S. Martoiu ${ }^{28 b}$, A.C. Martyniuk ${ }^{81}$, A. Marzin ${ }^{32}$, L. Masetti ${ }^{86}$, T. Mashimo ${ }^{157}$, R. Mashinistov ${ }^{98}$, J. Masik ${ }^{87}$, A.L. Maslennikov ${ }^{111, c}$, L.H. Mason ${ }^{91}$, L. Massa ${ }^{135 a, 135 b}$, P. Mastrandrea ${ }^{5}$, A. Mastroberardino ${ }^{40 a, 40 b}$, T. Masubuchi ${ }^{157}$, P. Mättig ${ }^{177}$, J. Maurer ${ }^{28 b}$, S.J. Maxfield ${ }^{77}$, 
D.A. Maximov ${ }^{111, c}$, R. Mazini ${ }^{153}$, I. Maznas ${ }^{156}$, S.M. Mazza ${ }^{139}$, N.C. Mc Fadden ${ }^{107}$, G. Mc Goldrick ${ }^{161}$, S.P. Mc Kee ${ }^{92}$, A. $\mathrm{McCarn}^{92}$, T.G. McCarthy ${ }^{103}$, L.I. McClymont ${ }^{81}$, E.F. McDonald ${ }^{91}$, J.A. Mcfayden ${ }^{32}$, G. Mchedlidze ${ }^{58}$, M.A. McKay ${ }^{43}$, K.D. McLean ${ }^{172}$, S.J. McMahon ${ }^{133}$, P.C. McNamara ${ }^{91}$, C.J. $\mathrm{McNicol}^{173}$, R.A. McPherson ${ }^{172, m}$, J.E. Mdhluli ${ }^{147 c}$, Z.A. Meadows ${ }^{89}$, S. Meehan ${ }^{140}$, T. Megy ${ }^{51}$, S. Mehlhase ${ }^{102}$, A. Mehta ${ }^{77}$, T. Meideck ${ }^{57}$, B. Meirose ${ }^{44}$, D. Melini ${ }^{170, a j}$, B.R. Mellado Garcia ${ }^{147 c}$, J.D. Mellenthin ${ }^{58}$, M. Melo ${ }^{146 a}$, F. Meloni ${ }^{18}$, A. Melzer ${ }^{23}$, S.B. Menary ${ }^{87}$, L. Meng ${ }^{77}$, X.T. Meng ${ }^{92}$, A. Mengarelli ${ }^{22 a, 22 b}$, S. Menke ${ }^{103}$, E. Meoni ${ }^{40 a}{ }^{40 b}$, S. Mergelmeyer ${ }^{17}$, C. Merlassino ${ }^{18}$, P. Mermod ${ }^{52}$, L. Merola ${ }^{106 a, 106 b}$, C. Meroni ${ }^{94 a}$, F.S. Merritt ${ }^{33}$, A. Messina ${ }^{134 a, 134 b}$, J. Metcalfe ${ }^{6}$, A.S. Mete ${ }^{166}$, C. Meyer ${ }^{124}$, J-P. Meyer ${ }^{138}$, J. Meyer ${ }^{154}$, H. Meyer Zu Theenhausen ${ }^{60 a}$, F. Miano ${ }^{151}$,

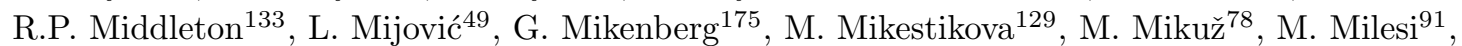
A. Milic ${ }^{161}$, D.A. Millar ${ }^{79}$, D.W. Miller ${ }^{33}$, A. Milov ${ }^{175}$, D.A. Milstead ${ }^{148 a, 148 b}$, A.A. Minaenko ${ }^{132}$, I.A. Minashvili ${ }^{54 b}$, A.I. Mincer ${ }^{112}$, B. Mindur ${ }^{41 a}$, M. Mineev ${ }^{68}$, Y. Minegishi ${ }^{157}$, Y. Ming ${ }^{176}$, L.M. Mir ${ }^{13}$,A. Mirto ${ }^{76 a, 76 b}$, K.P. Mistry ${ }^{124}$, T. Mitani ${ }^{174}$, J. Mitrevski ${ }^{102}$, V.A. Mitsou ${ }^{170}$, A. Miucci ${ }^{18}$, P.S. Miyagawa ${ }^{141}$, A. Mizukami ${ }^{69}$, J.U. Mjörnmark ${ }^{84}$, T. Mkrtchyan ${ }^{180}$, M. Mlynarikova ${ }^{131}$, T. Moa ${ }^{148 a, 148 b}$, K. Mochizuki ${ }^{97}$, P. $\operatorname{Mogg}^{51}$, S. Mohapatra ${ }^{38}$, S. Molander ${ }^{148 a, 148 b}$, R. Moles-Valls ${ }^{23}$, M.C. Mondragon ${ }^{93}$, K. Mönig ${ }^{45}$, J. Monk ${ }^{39}$, E. Monnier ${ }^{88}$, A. Montalbano ${ }^{144}$, J. Montejo Berlingen ${ }^{32}$, F. Monticelli ${ }^{74}$, S. Monzani ${ }^{94 a}$, R.W. Moore ${ }^{3}$, N. Morange ${ }^{119}$, D. Moreno ${ }^{21}$, M. Moreno Llácer ${ }^{32}$, P. Morettini ${ }^{53 a}$, M. Morgenstern ${ }^{109}$, S. Morgenstern ${ }^{32}$, D. Mori ${ }^{144}$, T. Mori ${ }^{157}$, M. Morii ${ }^{59}$, M. Morinaga ${ }^{174}$, V. Morisbak ${ }^{121}$, A.K. Morley ${ }^{32}$, G. Mornacchi ${ }^{32}$, J.D. Morris ${ }^{79}$, L. Morvaj ${ }^{150}$, P. Moschovakos ${ }^{10}$, M. Mosidze ${ }^{54 b}$, H.J. Moss ${ }^{141}$, J. Moss ${ }^{145, a k}$, K. Motohashi ${ }^{159}$, R. Mount ${ }^{145}$, E. Mountricha ${ }^{27}$, E.J.W. Moyse ${ }^{89}$, S. Muanza ${ }^{88}$, F. Mueller ${ }^{103}$, J. Mueller ${ }^{127}$, R.S.P. Mueller ${ }^{102}$, D. Muenstermann ${ }^{75}$, P. Mullen ${ }^{56}$, G.A. Mullier ${ }^{18}$, F.J. Munoz Sanchez ${ }^{87}$, P. Murin ${ }^{146 b}$, W.J. Murray ${ }^{173,133}$, A. Murrone ${ }^{94 a, 94 b}$, M. Muškinja ${ }^{78}$, C. Mwewa ${ }^{147 a}$, A.G. Myagkov ${ }^{132, a l}$, J. Myers ${ }^{118}$, M. Myska ${ }^{130}$, B.P. Nachman ${ }^{16}$, O. Nackenhorst ${ }^{46}$, K. Nagai ${ }^{122}$, R. Nagai ${ }^{69, a f}$, K. Nagano ${ }^{69}$, Y. Nagasaka ${ }^{61}$, K. Nagata ${ }^{164}$, M. Nagel ${ }^{51}$, E. Nagy ${ }^{88}$, A.M. Nairz ${ }^{32}$, Y. Nakahama ${ }^{105}$, K. Nakamura ${ }^{69}$, T. Nakamura ${ }^{157}$, I. Nakano ${ }^{114}$, F. Napolitano ${ }^{60 a}$, R.F. Naranjo Garcia ${ }^{45}$, R. Narayan ${ }^{11}$, D.I. Narrias Villar ${ }^{60 a}$, I. Naryshkin ${ }^{125}$, T. Naumann ${ }^{45}$, G. Navarro ${ }^{21}$, R. Nayyar ${ }^{7}$, H.A. Neal ${ }^{92}$, P.Yu. Nechaeva ${ }^{98}$, T.J. Neep ${ }^{138}$, A. Negri ${ }^{123 a, 123 b}$, M. Negrini ${ }^{22 a}$, S. Nektarijevic ${ }^{108}$, C. Nellist ${ }^{58}$, M.E. Nelson ${ }^{122}$, S. Nemecek ${ }^{129}$, P. Nemethy ${ }^{112}$, M. Nessi ${ }^{32, a m}$, M.S. Neubauer ${ }^{169}$, M. Neumann ${ }^{177}$, P.R. Newman ${ }^{19}$, T.Y. $\mathrm{Ng}^{62 c}$, Y.S. Ng${ }^{17}$, H.D.N. Nguyen ${ }^{88}$, T. Nguyen $\mathrm{Manh}^{97}$, E. Nibigira ${ }^{37}$, R.B. Nickerson ${ }^{122}$, R. Nicolaidou ${ }^{138}$, J. Nielsen ${ }^{139}$, N. Nikiforou ${ }^{11}$, V. Nikolaenko ${ }^{132, a l}$, I. Nikolic-Audit ${ }^{83}$, K. Nikolopoulos ${ }^{19}$, P. Nilsson ${ }^{27}$, Y. Ninomiya ${ }^{69}$, A. Nisati ${ }^{134 a}$, N. Nishu ${ }^{36 c}$,

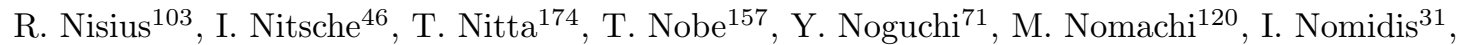
M.A. Nomura ${ }^{27}$, T. Nooney ${ }^{79}$, M. Nordberg ${ }^{32}$, N. Norjoharuddeen ${ }^{122}$, T. Novak ${ }^{78}$,

O. Novgorodova ${ }^{47}$, R. Novotny ${ }^{130}$, M. Nozaki ${ }^{69}$, L. Nozka ${ }^{117}$, K. Ntekas ${ }^{166}$, E. Nurse $^{81}$, F. Nuti ${ }^{91}$, K. O'Connor ${ }^{25}$, D.C. O'Neil ${ }^{144}$, A.A. O'Rourke ${ }^{45}$, V. O'Shea ${ }^{56}$, F.G. Oakham ${ }^{31, d}$, H. Oberlack ${ }^{103}$, T. Obermann ${ }^{23}$, J. Ocariz ${ }^{83}$, A. Ochi ${ }^{70}$, I. Ochoa ${ }^{38}$, J.P. Ochoa-Ricoux ${ }^{34 a}$, S. Oda ${ }^{73}$, S. Odaka $^{69}$, A. $\mathrm{Oh}^{87}$, S.H. $\mathrm{Oh}^{48}$, C.C. $\mathrm{Ohm}^{149}$, H. Ohman ${ }^{168}$, H. Oide ${ }^{53 a, 53 b}$, H. Okawa ${ }^{164}$, Y. Okazaki ${ }^{71}$, Y. Okumura ${ }^{157}$, T. Okuyama ${ }^{69}$, A. Olariu ${ }^{28 b}$, L.F. Oleiro Seabra ${ }^{128 a}$, S.A. Olivares Pino ${ }^{34 a}$, D. Oliveira Damazio ${ }^{27}$, J.L. Oliver ${ }^{1}$, M.J.R. Olsson ${ }^{33}$, A. Olszewski ${ }^{42}$, J. Olszowska ${ }^{42}$, A. Onofre ${ }^{128 a}, 128 \mathrm{e}$, K. Onogi ${ }^{105}$, P.U.E. Onyisi ${ }^{11, a n}$, H. Oppen ${ }^{121}$, M.J. Oreglia ${ }^{33}$, Y. Oren ${ }^{155}$, D. Orestano ${ }^{136 a, 136 b}$, E.C. Orgill ${ }^{87}$, N. Orlando ${ }^{62 b}$, R.S. Orr ${ }^{161}$, B. Osculati ${ }^{53 a, 53 b, *}$,

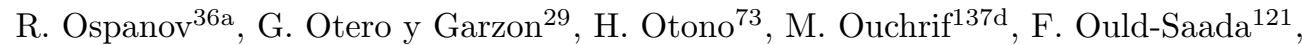
A. Ouraou ${ }^{138}$, Q. Ouyang ${ }^{35 a}$, M. Owen ${ }^{56}$, R.E. Owen ${ }^{19}$, V.E. Ozcan ${ }^{20 a}$, N. Ozturk ${ }^{8}$, K. Pachal ${ }^{144}$, A. Pacheco Pages ${ }^{13}$, L. Pacheco Rodriguez ${ }^{138}$, C. Padilla Aranda ${ }^{13}$, S. Pagan Griso ${ }^{16}$, M. Paganini ${ }^{179}$, G. Palacino ${ }^{64}$, S. Palazzo ${ }^{40 a, 40 b}$, S. Palestini ${ }^{32}$, M. Palka ${ }^{41 b}$, D. Pallin ${ }^{37}$, 
I. Panagoulias ${ }^{10}$, C.E. Pandini ${ }^{52}$, J.G. Panduro Vazquez ${ }^{80}$, P. Pani ${ }^{32}$, L. Paolozzi ${ }^{52}$, Th.D. Papadopoulou ${ }^{10}$, K. Papageorgiou ${ }^{9, s}$, A. Paramonov ${ }^{6}$, D. Paredes Hernandez ${ }^{62 b}$, B. Parida ${ }^{36 c}$, A.J. Parker ${ }^{75}$, M.A. Parker ${ }^{30}$, K.A. Parker ${ }^{45}$, F. Parodi ${ }^{53 a, 53 b}$, J.A. Parsons ${ }^{38}$, U. Parzefall ${ }^{51}$, V.R. Pascuzzi ${ }^{161}$, J.M.P Pasner ${ }^{139}$, E. Pasqualucci ${ }^{134 a}$, S. Passaggio ${ }^{53 a}$, Fr. Pastore $^{80}$, P. Pasuwan ${ }^{148 a, 148 b}$, S. Pataraia ${ }^{86}$, J.R. Pater ${ }^{87}$, A. Pathak ${ }^{176, e}$, T. Pauly ${ }^{32}$, B. Pearson ${ }^{103}$, S. Pedraza Lopez ${ }^{170}$, R. Pedro ${ }^{128 a, 128 b}$, S.V. Peleganchuk ${ }^{111, c}$, O. Penc ${ }^{129}$, C. Peng ${ }^{35 a, 35 d}$, H. Peng ${ }^{36 a}$, J. Penwell ${ }^{64}$, B.S. Peralva ${ }^{26 b}$, M.M. Perego ${ }^{138}$, A.P. Pereira Peixoto ${ }^{128 a}$, D.V. Perepelitsa ${ }^{27}$, F. Peri ${ }^{17}$, L. Perini ${ }^{94 a, 94 b}$, H. Pernegger ${ }^{32}$, S. Perrella ${ }^{106 a, 106 b}$, V.D. Peshekhonov ${ }^{68, *}$, K. Peters ${ }^{45}$, R.F.Y. Peters ${ }^{87}$, B.A. Petersen ${ }^{32}$, T.C. Petersen ${ }^{39}$, E. Petit ${ }^{57}$, A. Petridis ${ }^{1}$, C. Petridou ${ }^{156}$, P. Petroff ${ }^{119}$, E. Petrolo ${ }^{134 a}$, M. Petrov ${ }^{122}$, F. Petrucci ${ }^{136 a, 136 b}$, N.E. Pettersson ${ }^{89}$, A. Peyaud ${ }^{138}$, R. Pezoa ${ }^{34 b}$, T. Pham ${ }^{91}$, F.H. Phillips ${ }^{93}$, P.W. Phillips ${ }^{133}$, G. Piacquadio ${ }^{150}$, E. Pianori ${ }^{173}$, A. Picazio ${ }^{89}$, M.A. Pickering ${ }^{122}$, R. Piegaia ${ }^{29}$, J.E. Pilcher ${ }^{33}$, A.D. Pilkington ${ }^{87}$, M. Pinamonti ${ }^{135 a, 135 b}$, J.L. Pinfold ${ }^{3}$, M. Pitt ${ }^{175}$, M.-A. Pleier ${ }^{27}$, V. Pleskot ${ }^{131}$, E. Plotnikova ${ }^{68}$, D. Pluth ${ }^{67}$, P. Podberezko ${ }^{111}$, R. Poettgen ${ }^{84}$, R. Poggi ${ }^{123 a, 123 b}$, L. Poggioli ${ }^{119}$, I. Pogrebnyak ${ }^{93}$, D. Pohl ${ }^{23}$, I. Pokharel ${ }^{58}$, G. Polesello ${ }^{123 a}$, A. Poley ${ }^{45}$, A. Policicchio ${ }^{40 a, 40 b}$, R. Polifka ${ }^{32}$, A. Polini ${ }^{22 a}$, C.S. Pollard ${ }^{45}$, V. Polychronakos ${ }^{27}$, D. Ponomarenko ${ }^{100}$, L. Pontecorvo ${ }^{134 a}$, G.A. Popeneciu ${ }^{28 d}$, D.M. Portillo Quintero ${ }^{83}$, S. Pospisil ${ }^{130}$, K. Potamianos ${ }^{45}$, I.N. Potrap ${ }^{68}$, C.J. Potter ${ }^{30}$, H. Potti ${ }^{11}$, T. Poulsen ${ }^{84}$, J. Poveda ${ }^{32}$, M.E. Pozo Astigarraga ${ }^{32}$, P. Pralavorio ${ }^{88}$, S. Prell ${ }^{67}$, D. Price ${ }^{87}$, M. Primavera ${ }^{76 a}$,

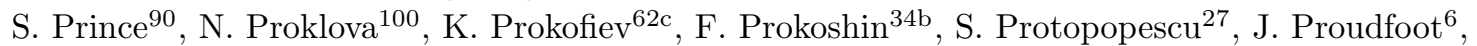
M. Przybycien ${ }^{41 a}$, A. Puri ${ }^{169}$, P. Puzo ${ }^{119}$, J. Qian ${ }^{92}$, Y. Qin ${ }^{87}$, A. Quadt ${ }^{58}$, M. Queitsch-Maitland ${ }^{45}$, A. Qureshi ${ }^{1}$, S.K. Radhakrishnan ${ }^{150}$, P. Rados ${ }^{91}$, F. Ragusa ${ }^{94 a, 94 b}$, G. Rahal ${ }^{181}$, J.A. Raine ${ }^{87}$, S. Rajagopalan ${ }^{27}$, T. Rashid ${ }^{119}$, S. Raspopov ${ }^{5}$, M.G. Ratti ${ }^{94 a, 94 b}$, D.M. Rauch ${ }^{45}$, F. Rauscher ${ }^{102}$, S. Rave ${ }^{86}$, B. Ravina ${ }^{141}$, I. Ravinovich ${ }^{175}$, J.H. Rawling ${ }^{87}$, M. Raymond ${ }^{32}$, A.L. Read ${ }^{121}$, N.P. Readioff ${ }^{57}$, M. Reale ${ }^{76 a, 76 b}$, D.M. Rebuzzi ${ }^{123 a, 123 b}$, A. Redelbach ${ }^{178}$, G. Redlinger ${ }^{27}$, R. Reece ${ }^{139}$, R.G. Reed ${ }^{147 c}$, K. Reeves ${ }^{44}$, L. Rehnisch $^{17}$, J. Reichert ${ }^{124}$, A. Reiss ${ }^{86}$, C. Rembser ${ }^{32}$, H. Ren ${ }^{35 a, 35 d}$, M. Rescigno ${ }^{134 a}$, S. Resconi ${ }^{94 a}$, E.D. Resseguie ${ }^{124}$, S. Rettie ${ }^{171}$, E. Reynolds ${ }^{19}$, O.L. Rezanova ${ }^{111, c}$, P. Reznicek ${ }^{131}$, R. Richter ${ }^{103}$, S. Richter ${ }^{81}$, E. Richter-Was ${ }^{41 b}$, O. Ricken ${ }^{23}$, M. Ridel ${ }^{83}$, P. Rieck ${ }^{103}$, C.J. Riegel ${ }^{177}$, O. Rifki ${ }^{45}$, M. Rijssenbeek ${ }^{150}$, A. Rimoldi ${ }^{123 a, 123 b}$, M. Rimoldi ${ }^{18}$, L. Rinaldi ${ }^{22 a}$, G. Ripellino ${ }^{149}$, B. Ristić ${ }^{32}$, E. Ritsch ${ }^{32}$, I. Riu ${ }^{13}$, J.C. Rivera Vergara ${ }^{34 a}$, F. Rizatdinova ${ }^{116}$, E. Rizvi ${ }^{79}$, C. Rizzi ${ }^{13}$, R.T. Roberts ${ }^{87}$, S.H. Robertson ${ }^{90, m}$, A. Robichaud-Veronneau ${ }^{90}$, D. Robinson ${ }^{30}$, J.E.M. Robinson ${ }^{45}$, A. Robson ${ }^{56}$, E. Rocco ${ }^{86}$, C. Roda ${ }^{126 a, 126 b}$, Y. Rodina ${ }^{88, a o}$, S. Rodriguez Bosca ${ }^{170}$, A. Rodriguez Perez ${ }^{13}$, D. Rodriguez Rodriguez ${ }^{170}$, A.M. Rodríguez Vera ${ }^{163 b}$, S. Roe ${ }^{32}$, C.S. Rogan ${ }^{59}$, O. Røhne ${ }^{121}$, R. Röhrig ${ }^{103}$, C.P.A. Roland ${ }^{64}$, J. Roloff ${ }^{59}$, A. Romaniouk ${ }^{100}$, M. Romano ${ }^{22 a, 22 b}$, E. Romero Adam $^{170}$, N. Rompotis ${ }^{77}$, M. Ronzani ${ }^{112}$, L. $\operatorname{Roos}^{83}$, S. Rosati ${ }^{134 a}$, K. Rosbach ${ }^{51}$, P. Rose ${ }^{139}$, N.-A. Rosien ${ }^{58}$, E. Rossi ${ }^{106 a}, 106 \mathrm{~b}$, L.P. Rossi ${ }^{53 a}$, L. Rossini ${ }^{94 a}$,94b, J.H.N. Rosten ${ }^{30}$, R. Rosten ${ }^{140}$, M. Rotaru ${ }^{28 b}$, J. Rothberg ${ }^{140}$, D. Rousseau ${ }^{119}$, D. Roy ${ }^{147 c}$, A. Rozanov ${ }^{88}$, Y. Rozen ${ }^{154}$, X. Ruan ${ }^{147 c}$, F. Rubbo ${ }^{145}$, F. Rühr ${ }^{51}$, A. Ruiz-Martinez ${ }^{31}$, Z. Rurikova ${ }^{51}$, N.A. Rusakovich ${ }^{68}$, H.L. Russell ${ }^{90}$, J.P. Rutherfoord ${ }^{7}$, N. Ruthmann ${ }^{32}$, E.M. Rüttinger ${ }^{45}$, Y.F. Ryabov ${ }^{125}$, M. Rybar ${ }^{169}$, G. Rybkin ${ }^{119}$, S. Ryu ${ }^{6}$, A. Ryzhov ${ }^{132}$, G.F. Rzehorz ${ }^{58}$, P. Sabatini ${ }^{58}$, G. Sabato ${ }^{109}$, S. Sacerdoti ${ }^{119}$, H.F-W. Sadrozinski ${ }^{139}$, R. Sadykov ${ }^{68}$, F. Safai Tehrani ${ }^{134 a}$, P. Saha ${ }^{110}$, M. Sahinsoy ${ }^{60 a}$, M. Saimpert ${ }^{45}$, M. Saito ${ }^{157}$, T. Saito ${ }^{157}$, H. Sakamoto ${ }^{157}$, A. Sakharov ${ }^{112}$, D. Salamani ${ }^{52}$, G. Salamanna ${ }^{136 a, 136 b}$, J.E. Salazar Loyola ${ }^{34 b}$, D. Salek ${ }^{109}$, P.H. Sales De Bruin ${ }^{168}$, D. Salihagic ${ }^{103}$, A. Salnikov ${ }^{145}$, J. Salt ${ }^{170}$, D. Salvatore ${ }^{40 a, 40 b}$, F. Salvatore ${ }^{151}$,

A. Salvucci ${ }^{62 a, 62 b, 62 c}$, A. Salzburger ${ }^{32}$, D. Sammel ${ }^{51}$, D. Sampsonidis ${ }^{156}$, D. Sampsonidou ${ }^{156}$, J. Sánchez ${ }^{170}$, A. Sanchez Pineda ${ }^{167 a, 167 c}$, H. Sandaker ${ }^{121}$, C.O. Sander ${ }^{45}$, M. Sandhoff ${ }^{177}$, 
C. Sandoval ${ }^{21}$, D.P.C. Sankey ${ }^{133}$, M. Sannino ${ }^{53 a, 53 b}$, Y. Sano ${ }^{105}$, A. Sansoni ${ }^{50}$, C. Santoni ${ }^{37}$, H. Santos ${ }^{128 a}$, I. Santoyo Castillo ${ }^{151}$, A. Sapronov ${ }^{68}$, J.G. Saraiva ${ }^{128 a, 128 e}$, O. Sasaki ${ }^{69}$, K. Sato ${ }^{164}$, E. Sauvan ${ }^{5}$, P. Savard ${ }^{161, d}$, N. Savic ${ }^{103}$, R. Sawada ${ }^{157}$, C. Sawyer ${ }^{133}$, L. Sawyer ${ }^{82, u}$, C. Sbarra ${ }^{22 a}$, A. Sbrizzi ${ }^{22 \mathrm{a}, 22 b}$, T. Scanlon ${ }^{81}$, D.A. Scannicchio ${ }^{166}$, J. Schaarschmidt ${ }^{140}$, P. Schacht ${ }^{103}$, B.M. Schachtner ${ }^{102}$, D. Schaefer ${ }^{33}$, L. Schaefer ${ }^{124}$, J. Schaeffer ${ }^{86}$, S. Schaepe ${ }^{32}$, U. Schäfer ${ }^{86}$, A.C. Schaffer ${ }^{119}$, D. Schaile ${ }^{102}$, R.D. Schamberger ${ }^{150}$, N. Scharmberg 87 , V.A. Schegelsky ${ }^{125}$, D. Scheirich ${ }^{131}$, F. Schenck ${ }^{17}$, M. Schernau ${ }^{166}$, C. Schiavi ${ }^{53 a, 53 b}$, S. Schier ${ }^{139}$, L.K. Schildgen ${ }^{23}$, Z.M. Schillaci ${ }^{25}$, E.J. Schioppa ${ }^{32}$, M. Schioppa ${ }^{40 a, 40 b}$, K.E. Schleicher ${ }^{51}$, S. Schlenker ${ }^{32}$, K.R. Schmidt-Sommerfeld ${ }^{103}$, K. Schmieden ${ }^{32}$, C. Schmitt ${ }^{86}$, S. Schmitt ${ }^{45}$, S. Schmitz ${ }^{86}$, U. Schnoor ${ }^{51}$, L. Schoeffel ${ }^{138}$, A. Schoening ${ }^{60 b}$, E. Schopf ${ }^{23}$, M. Schott ${ }^{86}$, J.F.P. Schouwenberg ${ }^{108}$, J. Schovancova ${ }^{32}$, S. Schramm ${ }^{52}$, N. Schuh ${ }^{86}$, A. Schulte ${ }^{86}$, H.-C. Schultz-Coulon ${ }^{60 a}$, M. Schumacher ${ }^{51}$, B.A. Schumm ${ }^{139}$, Ph. Schune ${ }^{138}$, A. Schwartzman ${ }^{145}$, T.A. Schwarz ${ }^{92}$, H. Schweiger ${ }^{87}$, Ph. Schwemling ${ }^{138}$, R. Schwienhorst ${ }^{93}$, A. Sciandra ${ }^{23}$, G. Sciolla ${ }^{25}$, M. Scornajenghi ${ }^{40 a, 40 b}$, F. Scuri ${ }^{126 a}$, F. Scutti ${ }^{91}$, L.M. Scyboz ${ }^{103}$, J. Searcy ${ }^{92}$, C.D. Sebastiani ${ }^{134 a, 134 b}$, P. Seema ${ }^{23}$, S.C. Seidel ${ }^{107}$, A. Seiden ${ }^{139}$, J.M. Seixas ${ }^{26 a}$, G. Sekhniaidze ${ }^{106 a}$, K. Sekhon ${ }^{92}$, S.J. Sekula ${ }^{43}$, N. Semprini-Cesari ${ }^{22 a, 22 b}$, S. Senkin ${ }^{37}$, C. Serfon ${ }^{121}$, L. Serin ${ }^{119}$, L. Serkin ${ }^{167 a, 167 b}$, M. Sessa ${ }^{136 a, 136 b}$, H. Severini ${ }^{115}$, T. Šfiligoj ${ }^{78}$, F. Sforza ${ }^{165}$, A. Sfyrla ${ }^{52}$, E. Shabalina ${ }^{58}$, J.D. Shahinian ${ }^{139}$, N.W. Shaikh ${ }^{148 a, 148 b}$, L.Y. Shan ${ }^{35 a}$, R. Shang ${ }^{169}$, J.T. Shank ${ }^{24}$, M. Shapiro ${ }^{16}$, A. Sharma ${ }^{122}$, A.S. Sharma ${ }^{1}$, P.B. Shatalov ${ }^{99}$, K. Shaw ${ }^{167 a, 167 b}$, S.M. Shaw ${ }^{87}$, A. Shcherbakova ${ }^{125}$, C.Y. Shehu ${ }^{151}$, Y. Shen ${ }^{115}$, N. Sherafati ${ }^{31}$, A.D. Sherman ${ }^{24}$, P. Sherwood ${ }^{81}$, L. Shi ${ }^{153, a p}$, S. Shimizu ${ }^{70}$, C.O. Shimmin ${ }^{179}$, M. Shimojima ${ }^{104}$, I.P.J. Shipsey ${ }^{122}$, S. Shirabe ${ }^{73}$, M. Shiyakova ${ }^{68, a q}$, J. Shlomi ${ }^{175}$, A. Shmeleva ${ }^{98}$,

D. Shoaleh Saadi ${ }^{97}$, M.J. Shochet ${ }^{33}$, S. Shojaii ${ }^{91}$, D.R. Shope ${ }^{115}$, S. Shrestha ${ }^{113}$, E. Shulga ${ }^{100}$, P. Sicho ${ }^{129}$, A.M. Sickles ${ }^{169}$, P.E. Sidebo ${ }^{149}$, E. Sideras Haddad ${ }^{147 c}$, O. Sidiropoulou ${ }^{178}$,

A. Sidoti ${ }^{22 a, 22 b}$, F. Siegert ${ }^{47}$, Dj. Sijacki ${ }^{14}$, J. Silva ${ }^{128 a, 128 e}$, M. Silva Jr. ${ }^{176}$, S.B. Silverstein ${ }^{148 a}$, L. Simic ${ }^{68}$, S. Simion ${ }^{119}$, E. Simioni ${ }^{86}$, B. Simmons ${ }^{81}$, M. Simon ${ }^{86}$, P. Sinervo ${ }^{161}$, N.B. Sinev ${ }^{118}$, M. Sioli ${ }^{22 a, 22 b}$, G. Siragusa ${ }^{178}$, I. Siral ${ }^{92}$, S.Yu. Sivoklokov ${ }^{101}$, J. Sjölin 148a,148b, M.B. Skinner ${ }^{75}$, P. Skubic ${ }^{115}$, M. Slater ${ }^{19}$, T. Slavicek ${ }^{130}$, M. Slawinska ${ }^{42}$, K. Sliwa ${ }^{165}$, R. Slovak ${ }^{131}$, V. Smakhtin ${ }^{175}$, B.H. Smart ${ }^{5}$, J. Smiesko ${ }^{146 a}$, N. Smirnov ${ }^{100}$, S.Yu. Smirnov ${ }^{100}$, Y. Smirnov ${ }^{100}$, L.N. Smirnova ${ }^{101, a r}$, O. Smirnova ${ }^{84}$, J.W. Smith ${ }^{58}$, M.N.K. Smith ${ }^{38}$, R.W. Smith ${ }^{38}$, M. Smizanska ${ }^{75}$, K. Smolek ${ }^{130}$, A.A. Snesarev ${ }^{98}$, I.M. Snyder ${ }^{118}$, S. Snyder ${ }^{27}$, R. Sobie ${ }^{172, m}$, F. Socher ${ }^{47}$, A.M. Soffa ${ }^{166}$, A. Soffer ${ }^{155}$, A. Søgaard ${ }^{49}$, D.A. Soh ${ }^{153}$, G. Sokhrannyi ${ }^{78}$, C.A. Solans Sanchez ${ }^{32}$, M. Solar ${ }^{130}$, E.Yu. Soldatov ${ }^{100}$, U. Soldevila ${ }^{170}$, A.A. Solodkov ${ }^{132}$, A. Soloshenko 68 , O.V. Solovyanov ${ }^{132}$, V. Solovyev ${ }^{125}$, P. Sommer ${ }^{141}$, H. Son ${ }^{165}$, W. Song ${ }^{133}$, A. Sopczak ${ }^{130}$, F. Sopkova ${ }^{146 b}$, D. Sosa ${ }^{60 b}$, C.L. Sotiropoulou ${ }^{126 a, 126 b}$, S. Sottocornola ${ }^{123 a, 123 b}$, R. Soualah ${ }^{167 a, 167 c}$, A.M. Soukharev ${ }^{111, c}$, D. South ${ }^{45}$, B.C. Sowden ${ }^{80}$, S. Spagnolo ${ }^{76 a, 76 b}$, M. Spalla ${ }^{103}$, M. Spangenberg ${ }^{173}$, F. Spanò ${ }^{80}$, D. Sperlich ${ }^{17}$, F. Spettel ${ }^{103}$, T.M. Spieker ${ }^{60 a}$, R. Spighi ${ }^{22 a}$, G. Spigo ${ }^{32}$, L.A. Spiller ${ }^{91}$, M. Spousta ${ }^{131}$, A. Stabile ${ }^{94 a, 94 b}$, R. Stamen ${ }^{60 a}$, S. Stamm ${ }^{17}$, E. Stanecka ${ }^{42}$, R.W. Stanek ${ }^{6}$, C. Stanescu ${ }^{136 a}$, M.M. Stanitzki ${ }^{45}$, B.S. Stapf ${ }^{109}$, S. Stapnes ${ }^{121}$, E.A. Starchenko ${ }^{132}$, G.H. Stark ${ }^{33}$, J. Stark ${ }^{57}$, S.H Stark ${ }^{39}$, P. Staroba ${ }^{129}$, P. Starovoitov ${ }^{60 a}$, S. Stärz ${ }^{32}$, R. Staszewski ${ }^{42}$, M. Stegler ${ }^{45}$, P. Steinberg ${ }^{27}$, B. Stelzer ${ }^{144}$, H.J. Stelzer ${ }^{32}$, O. Stelzer-Chilton ${ }^{163 a}$, H. Stenzel ${ }^{55}$, T.J. Stevenson ${ }^{79}$, G.A. Stewart ${ }^{32}$, M.C. Stockton ${ }^{118}$, G. Stoicea ${ }^{28 b}$, P. Stolte ${ }^{58}$, S. Stonjek ${ }^{103}$, A. Straessner ${ }^{47}$, J. Strandberg ${ }^{149}$, S. Strandberg ${ }^{148 a, 148 b}$, M. Strauss ${ }^{115}$, P. Strizenec ${ }^{146 b}$, R. Ströhmer ${ }^{178}$, D.M. Strom ${ }^{118}$, R. Stroynowski ${ }^{43}$, A. Strubig ${ }^{49}$, S.A. Stucci ${ }^{27}$, B. Stugu ${ }^{15}$, J. Stupak ${ }^{115}$, N.A. Styles ${ }^{45}$, D. Su ${ }^{145}$, J. Su ${ }^{127}$, S. Suchek ${ }^{60 a}$, Y. Sugaya ${ }^{120}$, M. Suk ${ }^{130}$, V.V. Sulin ${ }^{98}$, D.M.S. Sultan ${ }^{52}$, S. Sultansoy ${ }^{4 c}$, T. Sumida ${ }^{71}$, S. Sun ${ }^{92}$, X. Sun ${ }^{3}$, K. Suruliz ${ }^{151}$, C.J.E. Suster ${ }^{152}$, M.R. Sutton ${ }^{151}$, S. Suzuki ${ }^{69}$, M. Svatos ${ }^{129}$, M. Swiatlowski ${ }^{33}$, S.P. Swift ${ }^{2}$, A. Sydorenko ${ }^{86}$, I. Sykora ${ }^{146 a}$, T. Sykora ${ }^{131}$, D. Ta ${ }^{86}$, 
K. Tackmann ${ }^{45}$, J. Taenzer ${ }^{155}$, A. Taffard ${ }^{166}$, R. Tafirout ${ }^{163 a}$, E. Tahirovic ${ }^{79}$, N. Taiblum ${ }^{155}$, H. Takai ${ }^{27}$, R. Takashima ${ }^{72}$, E.H. Takasugi ${ }^{103}$, K. Takeda ${ }^{70}$, T. Takeshita ${ }^{142}$, Y. Takubo ${ }^{69}$, M. Talby ${ }^{88}$, A.A. Talyshev ${ }^{111, c}$, J. Tanaka ${ }^{157}$, M. Tanaka ${ }^{159}$, R. Tanaka ${ }^{119}$, R. Tanioka ${ }^{70}$, B.B. Tannenwald ${ }^{113}$, S. Tapia Araya ${ }^{34 b}$, S. Tapprogge ${ }^{86}$, A. Tarek Abouelfadl Mohamed ${ }^{83}$, S. Tarem ${ }^{154}$, G. Tarna ${ }^{28 b, p}$, G.F. Tartarelli ${ }^{94 a}$, P. Tas $^{131}$, M. Tasevsky ${ }^{129}$, T. Tashiro ${ }^{71}$,

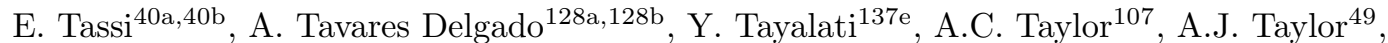
G.N. Taylor ${ }^{91}$, P.T.E. Taylor ${ }^{91}$, W. Taylor ${ }^{163 b}$, A.S. Tee ${ }^{75}$, P. Teixeira-Dias ${ }^{80}$, D. Temple ${ }^{144}$, H. Ten Kate ${ }^{32}$, P.K. Teng ${ }^{153}$, J.J. Teoh ${ }^{120}$, F. Tepel ${ }^{177}$, S. Terada ${ }^{69}$, K. Terashi ${ }^{157}$, J. Terron $^{85}$, S. Terzo ${ }^{13}$, M. Testa ${ }^{50}$, R.J. Teuscher ${ }^{161, m}$, S.J. Thais ${ }^{179}$, T. Theveneaux-Pelzer ${ }^{45}$, F. Thiele ${ }^{39}$, J.P. Thomas ${ }^{19}$, P.D. Thompson ${ }^{19}$, A.S. Thompson ${ }^{56}$, L.A. Thomsen ${ }^{179}$, E. Thomson ${ }^{124}$, Y. $\operatorname{Tian}^{38}$, R.E. Ticse Torres $^{58}$, V.O. Tikhomirov ${ }^{98, a s}$, Yu.A. Tikhonov ${ }^{111, c}$, S. Timoshenko ${ }^{100}$, P. Tipton ${ }^{179}$, S. Tisserant ${ }^{88}$, K. Todome ${ }^{159}$, S. Todorova-Nova ${ }^{5}$, S. Todt ${ }^{47}$, J. Tojo ${ }^{73}$, S. Tokár ${ }^{146 a}$, K. Tokushuku ${ }^{69}$, E. Tolley ${ }^{113}$, M. Tomoto ${ }^{105}$, L. Tompkins ${ }^{145, a t}$, K. Toms ${ }^{107}$, B. Tong ${ }^{59}$, P. Tornambe ${ }^{51}$, E. Torrence ${ }^{118}$, H. Torres ${ }^{47}$, E. Torró Pastor ${ }^{140}$, C. Tosciri ${ }^{122}$, J. Toth ${ }^{88, a u}$, F. Touchard ${ }^{88}$, D.R. Tovey ${ }^{141}$, C.J. Treado ${ }^{112}$, T. Trefzger ${ }^{178}$, F. Tresoldi ${ }^{151}$,

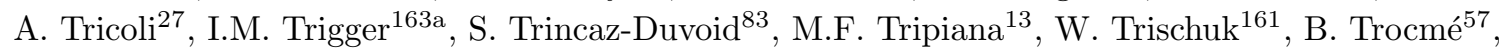
A. Trofymov ${ }^{45}$, C. Troncon ${ }^{94 a}$, M. Trovatelli ${ }^{172}$, F. Trovato ${ }^{151}$, L. Truong $^{147 b}$, M. Trzebinski ${ }^{42}$,

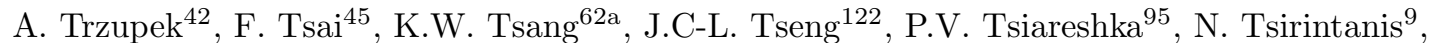
S. Tsiskaridze ${ }^{13}$, V. Tsiskaridze ${ }^{150}$, E.G. Tskhadadze ${ }^{54 a}$, I.I. Tsukerman ${ }^{99}$, V. Tsulaia ${ }^{16}$, S. Tsuno ${ }^{69}$, D. Tsybychev ${ }^{150}$, Y. Tu ${ }^{62 b}$, A. Tudorache ${ }^{28 b}$, V. Tudorache ${ }^{28 b}$, T.T. Tulbure ${ }^{28 a}$, A.N. Tuna ${ }^{59}$, S. Turchikhin ${ }^{68}$, D. Turgeman ${ }^{175}$, I. Turk Cakir ${ }^{4 b, a v}$, R. Turra ${ }^{94 a}$, P.M. Tuts ${ }^{38}$,

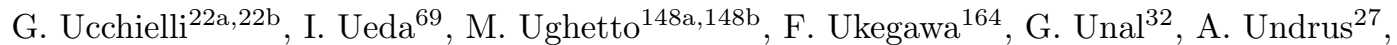

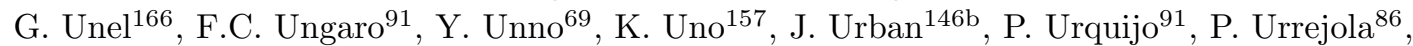

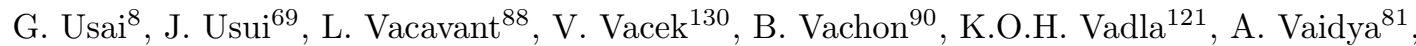
C. Valderanis ${ }^{102}$, E. Valdes Santurio ${ }^{148 a, 148 b}$, M. Valente ${ }^{52}$, S. Valentinetti ${ }^{22 a, 22 b}$, A. Valero ${ }^{170}$, L. Valéry ${ }^{45}$, R.A. Vallance ${ }^{19}$, A. Vallier ${ }^{5}$, J.A. Valls Ferrer ${ }^{170}$, T.R. Van Daalen ${ }^{13}$, W. Van Den Wollenberg ${ }^{109}$, H. van der Graaf ${ }^{109}$, P. van Gemmeren ${ }^{6}$, J. Van Nieuwkoop ${ }^{144}$, I. van Vulpen ${ }^{109}$, M.C. van Woerden ${ }^{109}$, M. Vanadia ${ }^{135 a, 135 b}$, W. Vandelli ${ }^{32}$, A. Vaniachine ${ }^{160}$, P. Vankov ${ }^{109}$, R. Vari ${ }^{134 a}$, E.W. Varnes ${ }^{7}$, C. Varni ${ }^{53 a, 53 b}$, T. Varol ${ }^{43}$, D. Varouchas ${ }^{119}$,

A. Vartapetian ${ }^{8}$, K.E. Varvell ${ }^{152}$, J.G. Vasquez ${ }^{179}$, G.A. Vasquez ${ }^{34 b}$, F. Vazeille ${ }^{37}$,

D. Vazquez Furelos ${ }^{13}$, T. Vazquez Schroeder ${ }^{90}$, J. Veatch ${ }^{58}$, V. Vecchio ${ }^{136 a, 136 b}$, L.M. Veloce ${ }^{161}$, F. Veloso ${ }^{128 a, 128 c}$, S. Veneziano ${ }^{134 a}$, A. Ventura ${ }^{76 a, 76 b}$, M. Venturi ${ }^{172}$, N. Venturi ${ }^{32}$, V. Vercesi ${ }^{123 a}$, M. Verducci ${ }^{136 a, 136 b}$, W. Verkerke ${ }^{109}$, A.T. Vermeulen ${ }^{109}$, J.C. Vermeulen ${ }^{109}$, M.C. Vetterli ${ }^{144, d}$, N. Viaux Maira ${ }^{34 b}$, O. Viazlo ${ }^{84}$, I. Vichou ${ }^{169, *}$, T. Vickey ${ }^{141}$, O.E. Vickey Boeriu ${ }^{141}$, G.H.A. Viehhauser ${ }^{122}$, S. Viel ${ }^{16}$, L. Vigani ${ }^{122}$, M. Villa ${ }^{22 a, 22 b}$, M. Villaplana Perez ${ }^{94 a, 94 b}$, E. Vilucchi ${ }^{50}$, M.G. Vincter ${ }^{31}$, V.B. Vinogradov ${ }^{68}$, A. Vishwakarma ${ }^{45}$, C. Vittori ${ }^{22 a, 22 b}$, I. Vivarelli ${ }^{151}$, S. Vlachos ${ }^{10}$, M. Vogel ${ }^{177}$, P. Vokac ${ }^{130}$, G. Volpi ${ }^{13}$, S.E. von Buddenbrock ${ }^{147 c}$, E. von Toerne ${ }^{23}$, V. Vorobel ${ }^{131}$, K. Vorobev ${ }^{100}$, M. Vos ${ }^{170}$, J.H. Vossebeld ${ }^{77}$, N. Vranjes ${ }^{14}$, M. Vranjes Milosavljevic ${ }^{14}$, V. Vrba ${ }^{130}$, M. Vreeswijk ${ }^{109}$, R. Vuillermet ${ }^{32}$, I. Vukotic ${ }^{33}$, P. Wagner ${ }^{23}$, W. Wagner ${ }^{177}$, J. Wagner-Kuhr ${ }^{102}$, H. Wahlberg ${ }^{74}$, S. Wahrmund $^{47}$, K. Wakamiya ${ }^{70}$, J. Walder ${ }^{75}$, R. Walker ${ }^{102}$, W. Walkowiak ${ }^{143}$, V. Wallangen ${ }^{148 a, 148 b}$, A.M. Wang ${ }^{59}$, C. Wang ${ }^{36 b, p}$, F. Wang ${ }^{176}$, H. Wang ${ }^{16}$, H. Wang ${ }^{3}$, J. Wang ${ }^{60 b}$, J. Wang ${ }^{152}$, P. Wang ${ }^{43}$, Q. Wang ${ }^{115}$,

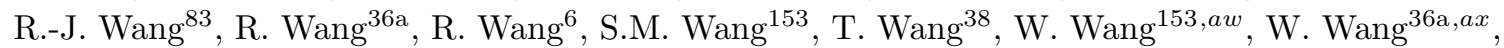

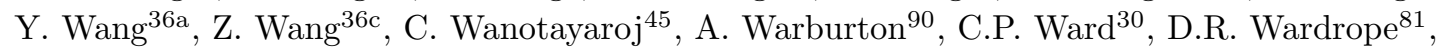
A. Washbrook ${ }^{49}$, P.M. Watkins ${ }^{19}$, A.T. Watson ${ }^{19}$, M.F. Watson ${ }^{19}$, G. Watts ${ }^{140}$, S. Watts ${ }^{87}$, B.M. Waugh ${ }^{81}$, A.F. Webb ${ }^{11}$, S. Webb ${ }^{86}$, C. Weber ${ }^{179}$, M.S. Weber ${ }^{18}$, S.M. Weber ${ }^{60 a}$, S.A. Weber ${ }^{31}$, J.S. Webster ${ }^{6}$, A.R. Weidberg ${ }^{122}$, B. Weinert ${ }^{64}$, J. Weingarten ${ }^{58}$, M. Weirich ${ }^{86}$, C. Weiser ${ }^{51}$, P.S. Wells ${ }^{32}$, T. Wenaus ${ }^{27}$, T. Wengler ${ }^{32}$, S. Wenig ${ }^{32}$, N. Wermes ${ }^{23}$, M.D. Werner ${ }^{67}$, 
P. Werner ${ }^{32}$, M. Wessels ${ }^{60 a}$, T.D. Weston ${ }^{18}$, K. Whalen ${ }^{118}$, N.L. Whallon ${ }^{140}$, A.M. Wharton ${ }^{75}$, A.S. White ${ }^{92}$, A. White ${ }^{8}$, M.J. White ${ }^{1}$, R. White ${ }^{34 b}$, D. Whiteson ${ }^{166}$, B.W. Whitmore ${ }^{75}$, F.J. Wickens ${ }^{133}$, W. Wiedenmann ${ }^{176}$, M. Wielers ${ }^{133}$, C. Wiglesworth ${ }^{39}$, L.A.M. Wiik-Fuchs ${ }^{51}$, A. Wildauer ${ }^{103}$, F. Wilk ${ }^{87}$, H.G. Wilkens ${ }^{32}$, H.H. Williams ${ }^{124}$, S. Williams ${ }^{30}$, C. Willis ${ }^{93}$, S. Willocq ${ }^{89}$, J.A. Wilson ${ }^{19}$, I. Wingerter-Seez ${ }^{5}$, E. Winkels ${ }^{151}$, F. Winklmeier ${ }^{118}$, O.J. Winston ${ }^{151}$, B.T. Winter ${ }^{23}$, M. Wittgen ${ }^{145}$, M. Wobisch ${ }^{82, u}$, A. Wolf ${ }^{86}$, T.M.H. Wolf ${ }^{109}$, R. Wolff ${ }^{88}$, M.W. Wolter ${ }^{42}$, H. Wolters ${ }^{128 a, 128 c}$, V.W.S. Wong ${ }^{171}$, N.L. Woods ${ }^{139}$, S.D. Worm ${ }^{19}$, B.K. Wosiek ${ }^{42}$, K.W. Woźniak ${ }^{42}$, K. Wraight ${ }^{56}$, M. Wu ${ }^{33}$, S.L. Wu ${ }^{176}, \mathrm{X} \mathrm{Wu}^{52}$, Y. Wu ${ }^{36 a}$, T.R. Wyatt ${ }^{87}$, B.M. Wynne ${ }^{49}$, S. Xella ${ }^{39}$, Z. Xi ${ }^{92}$, L. Xia ${ }^{35 c}$, D. Xu ${ }^{35 a}$, H. Xu ${ }^{36 a}$, L. Xu ${ }^{27}$, T. $\mathrm{Xu}^{138}$, W. $\mathrm{Xu}^{92}$, B. Yabsley ${ }^{152}$, S. Yacoob ${ }^{147 a}$, K. Yajima ${ }^{120}$, D.P. Yallup ${ }^{81}$, D. Yamaguchi ${ }^{159}$, Y. Yamaguchi ${ }^{159}$, A. Yamamoto ${ }^{69}$, T. Yamanaka ${ }^{157}$, F. Yamane ${ }^{70}$, M. Yamatani ${ }^{157}$,

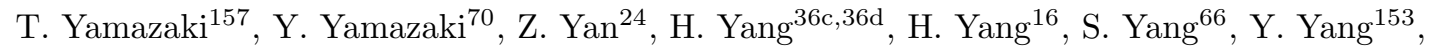
Y. Yang ${ }^{157}$, Z. Yang ${ }^{15}$, W-M. Yao ${ }^{16}$, Y.C. Yap ${ }^{45}$, Y. Yasu ${ }^{69}$, E. Yatsenko ${ }^{5}$, K.H. Yau Wong ${ }^{23}$, J. Ye ${ }^{43}$, S. $\mathrm{Ye}^{27}$, I. Yeletskikh ${ }^{68}$, E. Yigitbasi ${ }^{24}$, E. Yildirim ${ }^{86}$, K. Yorita ${ }^{174}$, K. Yoshihara ${ }^{124}$, C. Young ${ }^{145}$, C.J.S. Young ${ }^{32}$, J. Yu ${ }^{8}$, J. Yu ${ }^{67}$, X. Yue ${ }^{60 a}$, S.P.Y. Yuen ${ }^{23}$, I. Yusuff ${ }^{30, a y, ~}$ B. Zabinski ${ }^{42}$, G. Zacharis ${ }^{10}$, R. Zaidan ${ }^{13}$, A.M. Zaitsev ${ }^{132, a l}$, N. Zakharchuk ${ }^{45}$, J. Zalieckas ${ }^{15}$, S. Zambito ${ }^{59}$, D. Zanzi ${ }^{32}$, C. Zeitnitz ${ }^{177}$, G. Zemaityte ${ }^{122}$, J.C. Zeng ${ }^{169}$, Q. Zeng ${ }^{145}$, O. Zenin ${ }^{132}$, T. Ženišs ${ }^{146 a}$, D. Zerwas ${ }^{119}$, M. Zgubič ${ }^{122}$, D. Zhang ${ }^{36 \mathrm{~b}}$, D. Zhang ${ }^{92}$, F. Zhang ${ }^{176}$, G. Zhang ${ }^{36 a, a x}$, H. Zhang ${ }^{35 b}$, J. Zhang ${ }^{6}$, L. Zhang ${ }^{51}$, L. Zhang ${ }^{36 a}$, M. Zhang ${ }^{169}$, P. Zhang ${ }^{35 b}$, R. Zhang ${ }^{23}$, R. Zhang ${ }^{36 a, p}$, X. Zhang ${ }^{36 b}$, Y. Zhang ${ }^{35 a, 35 d}$, Z. Zhang ${ }^{119}$, X. Zhao ${ }^{43}$, Y. Zhao ${ }^{36 b, x}$, Z. Zhao ${ }^{36 a}$, A. Zhemchugov ${ }^{68}$, B. Zhou ${ }^{92}$, C. Zhou ${ }^{176}$, L. Zhou ${ }^{43}$, M. Zhou ${ }^{35 a, 35 d, ~ M . ~ Z h o u ~}{ }^{150}$, N. Zhou ${ }^{36 c}$, Y. Zhou ${ }^{7}$, C.G. Zhu ${ }^{36 \mathrm{~b}}$, H. Zhu ${ }^{36 \mathrm{a}}$, H. Zhu ${ }^{35 a}$, J. Zhu ${ }^{92}$, Y. Zhu ${ }^{36 a}$, X. Zhuang ${ }^{35 a}$, K. Zhukov ${ }^{98}$, V. Zhulanov ${ }^{111, a z}$, A. Zibell ${ }^{178}$, D. Zieminska ${ }^{64}$, N.I. Zimine ${ }^{68}$, S. Zimmermann ${ }^{51}$, Z. Zinonos ${ }^{103}$, M. Zinser ${ }^{86}$, M. Ziolkowski ${ }^{143}$, L. Živković ${ }^{14}$, G. Zobernig ${ }^{176}$, A. Zoccoli ${ }^{22 a, 22 b}$, K. Zoch ${ }^{58}$, T.G. Zorbas ${ }^{141}$, R. Zou ${ }^{33}$, M. zur Nedden ${ }^{17}$ and L. Zwalinski ${ }^{32}$

1 Department of Physics, University of Adelaide, Adelaide, Australia

2 Physics Department, SUNY Albany, Albany NY, United States of America

3 Department of Physics, University of Alberta, Edmonton AB, Canada

4 (a) Department of Physics, Ankara University, Ankara; ${ }^{(b)}$ Istanbul Aydin University, Istanbul; ${ }^{(c)}$ Division of Physics, TOBB University of Economics and Technology, Ankara, Turkey

5 LAPP, Univ. Grenoble Alpes, Univ. Savoie Mont Blanc, CNRS/IN2P3, Annecy, France

6 High Energy Physics Division, Argonne National Laboratory, Argonne IL, United States of America

7 Department of Physics, University of Arizona, Tucson AZ, United States of America

8 Department of Physics, The University of Texas at Arlington, Arlington TX, United States of America

9 Physics Department, National and Kapodistrian University of Athens, Athens, Greece

10 Physics Department, National Technical University of Athens, Zografou, Greece

11 Department of Physics, The University of Texas at Austin, Austin TX, United States of America

12 Institute of Physics, Azerbaijan Academy of Sciences, Baku, Azerbaijan

13 Institut de Física d'Altes Energies (IFAE), The Barcelona Institute of Science and Technology, Barcelona, Spain

14 Institute of Physics, University of Belgrade, Belgrade, Serbia

15 Department for Physics and Technology, University of Bergen, Bergen, Norway

16 Physics Division, Lawrence Berkeley National Laboratory and University of California, Berkeley CA, United States of America

17 Department of Physics, Humboldt University, Berlin, Germany

18 Albert Einstein Center for Fundamental Physics and Laboratory for High Energy Physics, University of Bern, Bern, Switzerland

19 School of Physics and Astronomy, University of Birmingham, Birmingham, United Kingdom 
20 (a) Department of Physics, Bogazici University, Istanbul; ${ }^{(b)}$ Department of Physics Engineering, Gaziantep University, Gaziantep; ${ }^{(d)}$ Istanbul Bilgi University, Faculty of Engineering and Natural Sciences, Istanbul; ${ }^{(e)}$ Bahcesehir University, Faculty of Engineering and Natural Sciences, Istanbul, Turkey

21 Centro de Investigaciones, Universidad Antonio Narino, Bogota, Colombia

22 (a) INFN Sezione di Bologna; ${ }^{(b)}$ Dipartimento di Fisica e Astronomia, Università di Bologna, Bologna, Italy

23 Physikalisches Institut, University of Bonn, Bonn, Germany

24 Department of Physics, Boston University, Boston MA, United States of America

25 Department of Physics, Brandeis University, Waltham MA, United States of America

26 (a) Universidade Federal do Rio De Janeiro COPPE/EE/IF, Rio de Janeiro; ${ }^{(b)}$ Electrical Circuits Department, Federal University of Juiz de Fora (UFJF), Juiz de Fora; ${ }^{(c)}$ Federal University of Sao Joao del Rei (UFSJ), Sao Joao del Rei; ${ }^{(d)}$ Instituto de Fisica, Universidade de Sao Paulo, Sao Paulo, Brazil

27 Physics Department, Brookhaven National Laboratory, Upton NY, United States of America

28 (a) Transilvania University of Brasov, Brasov; ${ }^{(b)}$ Horia Hulubei National Institute of Physics and Nuclear Engineering; ${ }^{(c)}$ Department of Physics, Alexandru Ioan Cuza University of Iasi, Iasi; ${ }^{(d)}$ National Institute for Research and Development of Isotopic and Molecular Technologies, Physics Department, Cluj Napoca; ${ }^{(e)}$ University Politehnica Bucharest, Bucharest; ${ }^{(f)}$ West University in Timisoara, Timisoara, Romania

29 Departamento de Física, Universidad de Buenos Aires, Buenos Aires, Argentina

30 Cavendish Laboratory, University of Cambridge, Cambridge, United Kingdom

31 Department of Physics, Carleton University, Ottawa ON, Canada

32 CERN, Geneva, Switzerland

33 Enrico Fermi Institute, University of Chicago, Chicago IL, United States of America

34 (a) Departamento de Física, Pontificia Universidad Católica de Chile, Santiago; ${ }^{(b)}$ Departamento de Física, Universidad Técnica Federico Santa María, Valparaíso, Chile

35 (a) Institute of High Energy Physics, Chinese Academy of Sciences, Beijing; ${ }^{(b)}$ Department of Physics, Nanjing University, Jiangsu; ${ }^{(c)}$ Physics Department, Tsinghua University, Beijing; ${ }^{(d)}$ University of Chinese Academy of Science (UCAS), Beijing, China

36 (a) Department of Modern Physics and State Key Laboratory of Particle Detection and Electronics, University of Science and Technology of China, Anhui; ${ }^{(b)}$ School of Physics, Shandong University, Shandong; (c) School of Physics and Astronomy, Key Laboratory for Particle Physics, Astrophysics and Cosmology, Ministry of Education; Shanghai Key Laboratory for Particle Physics and Cosmology, Shanghai Jiao Tong University; ${ }^{(d)}$ Tsung-Dao Lee Institute, Shanghai, China

37 Université Clermont Auvergne, CNRS/IN2P3, LPC, Clermont-Ferrand, France

38 Nevis Laboratory, Columbia University, Irvington NY, United States of America

39 Niels Bohr Institute, University of Copenhagen, Kobenhavn, Denmark

40 (a) INFN Gruppo Collegato di Cosenza, Laboratori Nazionali di Frascati; ${ }^{(b)}$ Dipartimento di Fisica, Università della Calabria, Rende, Italy

41 (a) AGH University of Science and Technology, Faculty of Physics and Applied Computer Science, Krakow; ${ }^{(b)}$ Marian Smoluchowski Institute of Physics, Jagiellonian University, Krakow, Poland

42 Institute of Nuclear Physics Polish Academy of Sciences, Krakow, Poland

43 Physics Department, Southern Methodist University, Dallas TX, United States of America

44 Physics Department, University of Texas at Dallas, Richardson TX, United States of America

45 DESY, Hamburg and Zeuthen, Germany

46 Lehrstuhl für Experimentelle Physik IV, Technische Universität Dortmund, Dortmund, Germany

47 Institut für Kern- und Teilchenphysik, Technische Universität Dresden, Dresden, Germany

48 Department of Physics, Duke University, Durham NC, United States of America

49 SUPA - School of Physics and Astronomy, University of Edinburgh, Edinburgh, United Kingdom

50 INFN e Laboratori Nazionali di Frascati, Frascati, Italy

51 Fakultät für Mathematik und Physik, Albert-Ludwigs-Universität, Freiburg, Germany 
Departement de Physique Nucleaire et Corpusculaire, Université de Genève, Geneva, Switzerland

53 (a) INFN Sezione di Genova; ${ }^{(b)}$ Dipartimento di Fisica, Università di Genova, Genova, Italy

54 (a) E. Andronikashvili Institute of Physics, Iv. Javakhishvili Tbilisi State University, Tbilisi; ${ }^{(b)}$ High Energy Physics Institute, Tbilisi State University, Tbilisi, Georgia

55 II. Physikalisches Institut, Justus-Liebig-Universität, Germany

56 SUPA - School of Physics and Astronomy, University of Glasgow, Glasgow, United Kingdom

57 LPSC, Université Grenoble Alpes, CNRS-IN2P3, Grenoble INP, Grenoble, France

58 II Physikalisches Institut, Georg-August-Universität, Göttingen, Germany

59 Laboratory for Particle Physics and Cosmology, Harvard University, Cambridge MA, United States of America

60 (a) Kirchhoff-Institut für Physik, Ruprecht-Karls-Universität Heidelberg, Heidelberg; ${ }^{(b)}$

Physikalisches Institut, Ruprecht-Karls-Universität Heidelberg, Heidelberg, Germany

61 Faculty of Applied Information Science, Hiroshima Institute of Technology, Hiroshima, Japan

62 (a) Department of Physics, The Chinese University of Hong Kong, Shatin, N.T., Hong Kong; ${ }^{(b)}$ Department of Physics, The University of Hong Kong, Hong Kong; ${ }^{(c)}$ Department of Physics and Institute for Advanced Study, The Hong Kong University of Science and Technology, Clear Water Bay, Kowloon, Hong Kong, China

63 Department of Physics, National Tsing Hua University, Hsinchu, Taiwan

64 Department of Physics, Indiana University, Bloomington IN, United States of America

${ }^{65}$ Institut für Astro- und Teilchenphysik, Leopold-Franzens-Universität, Innsbruck, Austria

66 University of Iowa, Iowa City IA, United States of America

67 Department of Physics and Astronomy, Iowa State University, Ames IA, United States of America

68 Joint Institute for Nuclear Research, JINR Dubna, Dubna, Russia

${ }^{69}$ KEK, High Energy Accelerator Research Organization, Tsukuba, Japan

${ }^{70}$ Graduate School of Science, Kobe University, Kobe, Japan

${ }^{71}$ Faculty of Science, Kyoto University, Kyoto, Japan

72 Kyoto University of Education, Kyoto, Japan

73 Research Center for Advanced Particle Physics and Department of Physics, Kyushu University, Fukuoka, Japan

74 Instituto de Física La Plata, Universidad Nacional de La Plata and CONICET, La Plata, Argentina

75 Physics Department, Lancaster University, Lancaster, United Kingdom

76 (a) INFN Sezione di Lecce; ${ }^{(b)}$ Dipartimento di Matematica e Fisica, Università del Salento, Lecce, Italy

77 Oliver Lodge Laboratory, University of Liverpool, Liverpool, United Kingdom

78 Department of Experimental Particle Physics, Jožef Stefan Institute and Department of Physics, University of Ljubljana, Ljubljana, Slovenia

79 School of Physics and Astronomy, Queen Mary University of London, London, United Kingdom

80 Department of Physics, Royal Holloway University of London, Surrey, United Kingdom

81 Department of Physics and Astronomy, University College London, London, United Kingdom

82 Louisiana Tech University, Ruston LA, United States of America

83 Laboratoire de Physique Nucléaire et de Hautes Energies, UPMC and Université Paris-Diderot and CNRS/IN2P3, Paris, France

84 Fysiska institutionen, Lunds universitet, Lund, Sweden

85 Departamento de Fisica Teorica C-15 and CIAFF, Universidad Autonoma de Madrid, Madrid, Spain

86 Institut für Physik, Universität Mainz, Mainz, Germany

87 School of Physics and Astronomy, University of Manchester, Manchester, United Kingdom

${ }_{88}$ CPPM, Aix-Marseille Université and CNRS/IN2P3, Marseille, France

89 Department of Physics, University of Massachusetts, Amherst MA, United States of America

90 Department of Physics, McGill University, Montreal QC, Canada

91 School of Physics, University of Melbourne, Victoria, Australia

92 Department of Physics, The University of Michigan, Ann Arbor MI, United States of America 
Department of Physics and Astronomy, Michigan State University, East Lansing MI, United States of America

$94{ }^{(a)}$ INFN Sezione di Milano; ${ }^{(b)}$ Dipartimento di Fisica, Università di Milano, Milano, Italy

95 B.I. Stepanov Institute of Physics, National Academy of Sciences of Belarus, Minsk, Republic of Belarus

96 Research Institute for Nuclear Problems of Byelorussian State University, Minsk, Republic of Belarus

97 Group of Particle Physics, University of Montreal, Montreal QC, Canada

${ }_{98}$ P.N. Lebedev Physical Institute of the Russian Academy of Sciences, Moscow, Russia

99 Institute for Theoretical and Experimental Physics (ITEP), Moscow, Russia

100 National Research Nuclear University MEPhI, Moscow, Russia

101 D.V. Skobeltsyn Institute of Nuclear Physics, M.V. Lomonosov Moscow State University, Moscow, Russia

102 Fakultät für Physik, Ludwig-Maximilians-Universität München, München, Germany

103 Max-Planck-Institut für Physik (Werner-Heisenberg-Institut), München, Germany

104 Nagasaki Institute of Applied Science, Nagasaki, Japan

105 Graduate School of Science and Kobayashi-Maskawa Institute, Nagoya University, Nagoya, Japan

106 (a) INFN Sezione di Napoli; ${ }^{(b)}$ Dipartimento di Fisica, Università di Napoli, Napoli, Italy

107 Department of Physics and Astronomy, University of New Mexico, Albuquerque NM, United States of America

108 Institute for Mathematics, Astrophysics and Particle Physics, Radboud University Nijmegen/Nikhef, Nijmegen, Netherlands

109 Nikhef National Institute for Subatomic Physics and University of Amsterdam, Amsterdam, Netherlands

110 Department of Physics, Northern Illinois University, DeKalb IL, United States of America

111 Budker Institute of Nuclear Physics, SB RAS, Novosibirsk, Russia

112 Department of Physics, New York University, New York NY, United States of America

113 Ohio State University, Columbus OH, United States of America

114 Faculty of Science, Okayama University, Okayama, Japan

115 Homer L. Dodge Department of Physics and Astronomy, University of Oklahoma, Norman OK, United States of America

116 Department of Physics, Oklahoma State University, Stillwater OK, United States of America

117 Palacký University, RCPTM, Olomouc, Czech Republic

118 Center for High Energy Physics, University of Oregon, Eugene OR, United States of America

119 LAL, Univ. Paris-Sud, CNRS/IN2P3, Université Paris-Saclay, Orsay, France

120 Graduate School of Science, Osaka University, Osaka, Japan

121 Department of Physics, University of Oslo, Oslo, Norway

122 Department of Physics, Oxford University, Oxford, United Kingdom

123 (a) INFN Sezione di Pavia; ${ }^{(b)}$ Dipartimento di Fisica, Università di Pavia, Pavia, Italy

124 Department of Physics, University of Pennsylvania, Philadelphia PA, United States of America

125 National Research Centre "Kurchatov Institute" B.P.Konstantinov Petersburg Nuclear Physics Institute, St. Petersburg, Russia

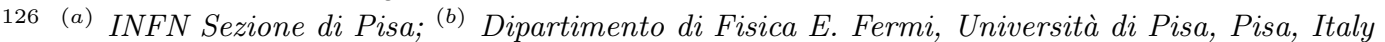

127 Department of Physics and Astronomy, University of Pittsburgh, Pittsburgh PA, United States of America

128 (a) Laboratório de Instrumentação e Física Experimental de Partículas - LIP, Lisboa; ${ }^{\left({ }^{b}\right)}$ Faculdade de Ciências, Universidade de Lisboa, Lisboa; (c) Department of Physics, University of Coimbra, Coimbra; ${ }^{(e)}$ Departamento de Fisica, Universidade do Minho, Braga, Portugal; ${ }^{(f)}$ Departamento de Fisica Teorica y del Cosmos, Universidad de Granada, Granada (Spain), Spain; ${ }^{(g)}$ Dep Fisica and CEFITEC of Faculdade de Ciencias e Tecnologia, Universidade Nova de Lisboa, Caparica, Portugal

129 Institute of Physics, Academy of Sciences of the Czech Republic, Praha, Czech Republic 
130 Czech Technical University in Prague, Praha, Czech Republic

131 Charles University, Faculty of Mathematics and Physics, Prague, Czech Republic

132 State Research Center Institute for High Energy Physics (Protvino), NRC KI, Russia

133 Particle Physics Department, Rutherford Appleton Laboratory, Didcot, United Kingdom

134 (a) INFN Sezione di Roma; ${ }^{(b)}$ Dipartimento di Fisica, Sapienza Università di Roma, Roma, Italy

135 (a) INFN Sezione di Roma Tor Vergata; ${ }^{(b)}$ Dipartimento di Fisica, Università di Roma Tor Vergata, Roma, Italy

136 (a) INFN Sezione di Roma Tre; ${ }^{(b)}$ Dipartimento di Matematica e Fisica, Università Roma Tre, Roma, Italy

137 (a) Faculté des Sciences Ain Chock, Réseau Universitaire de Physique des Hautes Energies Université Hassan II, Casablanca; ${ }^{(b)}$ Centre National de l'Energie des Sciences Techniques Nucleaires, Rabat; ${ }^{(c)}$ Faculté des Sciences Semlalia, Université Cadi Ayyad, LPHEA-Marrakech; (d) Faculté des Sciences, Université Mohamed Premier and LPTPM, Oujda; ${ }^{(e)}$ Faculté des sciences, Université Mohammed V, Rabat, Morocco

138 Institut de Recherches sur les Lois Fondamentales de l'Univers, DSM/IRFU, CEA Saclay, Gif-sur-Yvette, France

139 Santa Cruz Institute for Particle Physics, University of California Santa Cruz, Santa Cruz CA, United States of America

140 Department of Physics, University of Washington, Seattle WA, United States of America

141 Department of Physics and Astronomy, University of Sheffield, Sheffield, United Kingdom

142 Department of Physics, Shinshu University, Nagano, Japan

143 Department Physik, Universität Siegen, Siegen, Germany

144 Department of Physics, Simon Fraser University, Burnaby BC, Canada

145 SLAC National Accelerator Laboratory, Stanford CA, United States of America

146 (a) Faculty of Mathematics, Physics and Informatics, Comenius University, Bratislava; ${ }^{(b)}$ Department of Subnuclear Physics, Institute of Experimental Physics of the Slovak Academy of Sciences, Kosice, Slovak Republic

147 (a) Department of Physics, University of Cape Town, Cape Town; ${ }^{(b)}$ Department of Mechanical Engineering Science, University of Johannesburg, Johannesburg; ${ }^{(c)}$ School of Physics, University of the Witwatersrand, Johannesburg, South Africa

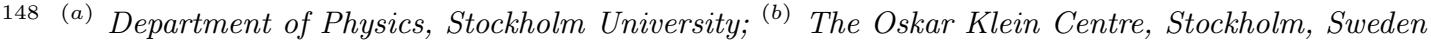

149 Physics Department, Royal Institute of Technology, Stockholm, Sweden

150 Departments of Physics and Astronomy, Stony Brook University, Stony Brook NY, United States of America

151 Department of Physics and Astronomy, University of Sussex, Brighton, United Kingdom

152 School of Physics, University of Sydney, Sydney, Australia

153 Institute of Physics, Academia Sinica, Taipei, Taiwan

154 Department of Physics, Technion: Israel Institute of Technology, Haifa, Israel

155 Raymond and Beverly Sackler School of Physics and Astronomy, Tel Aviv University, Tel Aviv, Israel

156 Department of Physics, Aristotle University of Thessaloniki, Thessaloniki, Greece

157 International Center for Elementary Particle Physics and Department of Physics, The University of Tokyo, Tokyo, Japan

158 Graduate School of Science and Technology, Tokyo Metropolitan University, Tokyo, Japan

159 Department of Physics, Tokyo Institute of Technology, Tokyo, Japan

160 Tomsk State University, Tomsk, Russia

161 Department of Physics, University of Toronto, Toronto ON, Canada

162 (a) INFN-TIFPA; ${ }^{(b)}$ University of Trento, Trento, Italy

163 (a) TRIUMF, Vancouver BC; ${ }^{(b)}$ Department of Physics and Astronomy, York University, Toronto ON, Canada

164 Division of Physics and Tomonaga Center for the History of the Universe, Faculty of Pure and Applied Sciences, University of Tsukuba, Tsukuba, Japan 
Department of Physics and Astronomy, Tufts University, Medford MA, United States of America

166 Department of Physics and Astronomy, University of California Irvine, Irvine CA, United States of America

167 (a) INFN Gruppo Collegato di Udine, Sezione di Trieste, Udine; ${ }^{(b)}$ ICTP, Trieste; ${ }^{(c)}$ Dipartimento di Chimica, Fisica e Ambiente, Università di Udine, Udine, Italy

168 Department of Physics and Astronomy, University of Uppsala, Uppsala, Sweden

169 Department of Physics, University of Illinois, Urbana IL, United States of America

170 Instituto de Fisica Corpuscular (IFIC), Centro Mixto Universidad de Valencia - CSIC, Spain

171 Department of Physics, University of British Columbia, Vancouver BC, Canada

172 Department of Physics and Astronomy, University of Victoria, Victoria BC, Canada

173 Department of Physics, University of Warwick, Coventry, United Kingdom

174 Waseda University, Tokyo, Japan

175 Department of Particle Physics, The Weizmann Institute of Science, Rehovot, Israel

176 Department of Physics, University of Wisconsin, Madison WI, United States of America

177 Fakultät für Mathematik und Naturwissenschaften, Fachgruppe Physik, Bergische Universität Wuppertal, Wuppertal, Germany

178 Fakultät für Physik und Astronomie, Julius-Maximilians-Universität, Würzburg, Germany

179 Department of Physics, Yale University, New Haven CT, United States of America

180 Yerevan Physics Institute, Yerevan, Armenia

181 Centre de Calcul de l'Institut National de Physique Nucléaire et de Physique des Particules (IN2P3), Villeurbanne, France

182 Academia Sinica Grid Computing, Institute of Physics, Academia Sinica, Taipei, Taiwan

a Also at Department of Physics, King's College London, London, United Kingdom

${ }^{b}$ Also at Institute of Physics, Azerbaijan Academy of Sciences, Baku, Azerbaijan

c Also at Novosibirsk State University, Novosibirsk, Russia

d Also at TRIUMF, Vancouver BC, Canada

e Also at Department of Physics and Astronomy, University of Louisville, Louisville, KY, United States of America

${ }^{f}$ Also at Department of Physics, California State University, Fresno CA, United States of America

$g$ Also at Department of Physics, University of Fribourg, Fribourg, Switzerland

$h$ Also at II Physikalisches Institut, Georg-August-Universität, Göttingen, Germany

${ }^{i}$ Also at Departament de Fisica de la Universitat Autonoma de Barcelona, Barcelona, Spain

${ }^{j}$ Also at Tomsk State University, Tomsk, and Moscow Institute of Physics and Technology State University, Dolgoprudny, Russia

${ }^{k}$ Also at The Collaborative Innovation Center of Quantum Matter (CICQM), Beijing, China

${ }^{l}$ Also at Universita di Napoli Parthenope, Napoli, Italy

$m$ Also at Institute of Particle Physics (IPP), Canada

${ }^{n}$ Also at Dipartimento di Fisica E. Fermi, Università di Pisa, Pisa, Italy

- Also at Horia Hulubei National Institute of Physics and Nuclear Engineering, Romania

${ }^{p}$ Also at CPPM, Aix-Marseille Université and CNRS/IN2P3, Marseille, France

$q$ Also at Department of Physics, St. Petersburg State Polytechnical University, St. Petersburg, Russia

${ }^{r}$ Also at Borough of Manhattan Community College, City University of New York, New York City, United States of America

$s$ Also at Department of Financial and Management Engineering, University of the Aegean, Chios, Greece

${ }^{t}$ Also at Centre for High Performance Computing, CSIR Campus, Rosebank, Cape Town, South Africa

u Also at Louisiana Tech University, Ruston LA, United States of America

$v$ Also at Institucio Catalana de Recerca i Estudis Avancats, ICREA, Barcelona, Spain

w Also at Department of Physics, The University of Michigan, Ann Arbor MI, United States of 
America

$x$ Also at LAL, Univ. Paris-Sud, CNRS/IN2P3, Université Paris-Saclay, Orsay, France

y Also at Graduate School of Science, Osaka University, Osaka, Japan

$z$ Also at Fakultät für Mathematik und Physik, Albert-Ludwigs-Universität, Freiburg, Germany

a Also at Institute for Mathematics, Astrophysics and Particle Physics, Radboud University Nijmegen/Nikhef, Nijmegen, Netherlands

ab Also at Near East University, Nicosia, North Cyprus, Mersin 10, Turkey

ac Also at Institute of Theoretical Physics, Ilia State University, Tbilisi, Georgia

ad Also at CERN, Geneva, Switzerland

ae Also at Georgian Technical University (GTU), Tbilisi, Georgia

af Also at Ochadai Academic Production, Ochanomizu University, Tokyo, Japan

ag Also at Manhattan College, New York NY, United States of America

ah Also at Hellenic Open University, Patras, Greece

ai Also at The City College of New York, New York NY, United States of America

aj Also at Departamento de Fisica Teorica y del Cosmos, Universidad de Granada, Granada (Spain), Spain

ak Also at Department of Physics, California State University, Sacramento CA, United States of America

al Also at Moscow Institute of Physics and Technology State University, Dolgoprudny, Russia

am Also at Departement de Physique Nucleaire et Corpusculaire, Université de Genève, Geneva, Switzerland

an Also at Department of Physics, The University of Texas at Austin, Austin TX, United States of America

ao Also at Institut de Física d'Altes Energies (IFAE), The Barcelona Institute of Science and Technology, Barcelona, Spain

ap Also at School of Physics, Sun Yat-sen University, Guangzhou, China

aq Also at Institute for Nuclear Research and Nuclear Energy (INRNE) of the Bulgarian Academy of Sciences, Sofia, Bulgaria

ar Also at Faculty of Physics, M.V.Lomonosov Moscow State University, Moscow, Russia

as Also at National Research Nuclear University MEPhI, Moscow, Russia

at Also at Department of Physics, Stanford University, Stanford CA, United States of America

au Also at Institute for Particle and Nuclear Physics, Wigner Research Centre for Physics, Budapest, Hungary

av Also at Giresun University, Faculty of Engineering, Turkey

aw Also at Department of Physics, Nanjing University, Jiangsu, China

ax Also at Institute of Physics, Academia Sinica, Taipei, Taiwan

ay Also at University of Malaya, Department of Physics, Kuala Lumpur, Malaysia

az Also at Budker Institute of Nuclear Physics, SB RAS, Novosibirsk, Russia

* Deceased 